\title{
Pressure Loss Modeling of Non-Symmetric Gas Turbine Exhaust Ducts using CFD
}

\author{
Steven Farber
}

\author{
A Thesis \\ in \\ The Department \\ of \\ Mechanical and Industrial Engineering \\ Presented in Partial Fulfillment of the Requirements \\ Concordia University \\ Montreal, Quebec, Canada
}

for the Degree of Master of Applied Science (Mechanical Engineering)at

April 15, 2008

(C) Steven Farber, 2008 


$\begin{array}{ll}\begin{array}{l}\text { Library and } \\ \text { Archives Canada }\end{array} & \begin{array}{l}\text { Bibliothèque et } \\ \text { Archives Canada }\end{array} \\ \begin{array}{l}\text { Published Heritage } \\ \text { Branch }\end{array} & \begin{array}{l}\text { Direction du } \\ \text { Patrimoine de l'édition }\end{array} \\ \begin{array}{l}\text { 395 Wellington Street } \\ \text { Ottawa ON K1A 0N4 } \\ \text { Canada }\end{array} & \begin{array}{l}\text { 395, rue Wellington } \\ \text { Ottawa ON K1A 0N4 } \\ \text { Canada }\end{array}\end{array}$

Your file Votre référence ISBN: 978-0-494-40910-7

Our file Notre référence

ISBN: 978-0-494-40910-7

NOTICE:

The author has granted a nonexclusive license allowing Library and Archives Canada to reproduce, publish, archive, preserve, conserve, communicate to the public by telecommunication or on the Internet, loan, distribute and sell theses worldwide, for commercial or noncommercial purposes, in microform, paper, electronic and/or any other formats.

The author retains copyright ownership and moral rights in this thesis. Neither the thesis nor substantial extracts from it may be printed or otherwise reproduced without the author's permission.
AVIS:

L'auteur a accordé une licence non exclusive permettant à la Bibliothèque et Archives Canada de reproduire, publier, archiver, sauvegarder, conserver, transmettre au public par télécommunication ou par l'Internet, prêter, distribuer et vendre des thèses partout dans le monde, à des fins commerciales ou autres, sur support microforme, papier, électronique et/ou autres formats.

L'auteur conserve la propriété du droit d'auteur et des droits moraux qui protège cette thèse. $\mathrm{Ni}$ la thèse ni des extraits substantiels de celle-ci ne doivent être imprimés ou autrement reproduits sans son autorisation.
In compliance with the Canadian Privacy Act some supporting forms may have been removed from this thesis.

While these forms may be included in the document page count, their removal does not represent any loss of content from the thesis.
Conformément à la loi canadienne sur la protection de la vie privée, quelques formulaires secondaires ont été enlevés de cette thèse.

Bien que ces formulaires aient inclus dans la pagination, il n'y aura aucun contenu manquant.

\section{Canada}




\begin{abstract}
Pressure Loss Modeling of Non-Symmetric Gas Turbine Exhaust Ducts using CFD

Steven Farber
\end{abstract}

In typical gas turbine applications, combustion gases that are discharged from the turbine are exhausted into the atmosphere in a direction that is sometimes different from that of the inlet. In such cases, the design of efficient exhaust ducts is a challenging task particularly when the exhaust gases are also swirling. Designers are in need for a tool today that can guide them in assessing qualitatively and quantitatively the different flow physics in these exhaust ducts so as to produce efficient designs.

In this thesis, a parametric Computational Fluid Dynamics (CFD) based study was carried out on non-symmetric gas turbine exhaust ducts where the effects of geometry and inlet aerodynamic conditions were examined. The results of the numerical analysis were used to develop a total pressure loss model.

These exhaust ducts comprise an annular inlet, a flow splitter, an annular to rectangular transition region, and an exhaust stub. The duct geometry, which is a three-dimensional complex one, is approximated with a five-parameter model, which was coupled with a design of experiment method to generate a relatively small number of exhaust ducts. The flow in these ducts was simulated using CFD for different values of inlet swirl and aerodynamic blockage and the numerical results were reviewed so as to assess the effects of the geometric and aerodynamic parameters on the total pressure loss in the exhaust duct. These flow simulations were used as a data base to generate a total pressure loss model that designers can use as a tool to build more efficient non-symmetric gas turbine exhaust ducts. The resulting correlation has demonstrated satisfactory agreement with the $\mathrm{CFD}$-based data. 


\section{Acknowledgments}

I would like to first thank my supervisor Prof. W.S. Ghaly and Co-supervisor Ed Vlasic for their guidance and suggestions on this project.

Thanks also go to the following people and organizations without which this work would not have been possibe:

To Pratt \& Whitney Canada for their financial and technical support, especially Remo Marini and Mark Cunningham.

To the Natural Sciences and Engineering Research Council of Canada (NSERC) for financial support. 


\section{Contents}

1 Introduction 1

1.1 Background .......................... 1

1.2 Single Port Annular-to-Rectangular Exhaust Ducts . . . . . . . . . . . 3

1.3 Contribution and Scope of the Present Study . . . . . . . . . . . . 4

2 Theory and Literature Review 6

2.1 Diffuser Performance . . . . . . . . . . . . . . . . . 6

2.1.1 Static Pressure Recovery Coefficient . . . . . . . . . . . . 6

2.1 .2 Diffuser Effectiveness . . . . . . . . . . . . 7

2.1.3 Total Pressure Loss Coefficient . . . . . . . . . . . 7

2.2 Conical Diffusers $\ldots \ldots \ldots \ldots \ldots \ldots \ldots$

2.2 .1 Geometry . . . . . . . . . . . . . . . 7

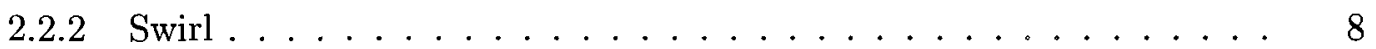

2.2 .3 Aerodynamic Blockage . . . . . . . . . . . . . . . . 9

2.3 Annular Diffusers . . . . . . . . . . . . . . . . . . . 11

$2.3 .1 \quad$ Swirl . . . . . . . . . . . . . . . . . . 14

2.3.2 Aerodynamic Blockage . . . . . . . . . . . . . . . . . 19

2.4 Past Research Contributions . . . . . . . . . . . . . . . . 21

2.4 .1 Loka et al. . . . . . . . . . . . . . . . . . 21

2.4 .2 Cunningham . . . . . . . . . . . . . . . . 24

3 Design of Experiment $\quad 30$

3.1 Geometric Design Space . . . . . . . . . . . . . . . . . 30 
3.1.1 Equivalent Cone Diffusion Angle . . . . . . . . . . . . . . . 31

3.1 .2 Flow Splitter Wedge Angle . . . . . . . . . . . . . . . . 31

3.1 .3 Gas Path Aspect Ratio . . . . . . . . . . . . . . . . 34

3.1.4 Annular to Rectangular Transition Region . . . . . . . . . . . . . 35

3.1 .5 Exhaust Stubs ....................... 36

3.2 Aerodynamic Design Space . . . . . . . . . . . . . . . 38

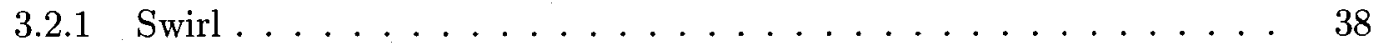

3.2 .2 Inlet Boundary Layer Blockage . . . . . . . . . . . . . . 40

3.3 Full Factorial Design . . . . . . . . . . . . . . . . . . 40

3.4 Taguchi Design . . . . . . . . . . . . . . . . . . 43

3.4 .1 Assumption . . . . . . . . . . . . . 43

3.4 .2 Interactions . . . . . . . . . . . . . 43

3.4.3 Selecting an Orthogonal Array ................ 44

4 Geometry Synthesis $\quad 47$

4.1 Equivalent Cone Diffusion Angle . . . . . . . . . . . . . . . . 49

4.2 Gas Path . . . . . . . . . . . . . . . . . . 51

4.3 Gas Path Aspect Ratio . . . . . . . . . . . . . . . . . . . . 51

4.4 Flow Splitter Leading Edge . . . . . . . . . . . . . . . 51

4.5 Flow Splitter Wedge Angle . . . . . . . . . . . . . . 54

4.6 Duct Exit Cross-Section . . . . . . . . . . . . . . . . 54

4.7 Annular to Rectangular Transition Region . . . . . . . . . . . . 57

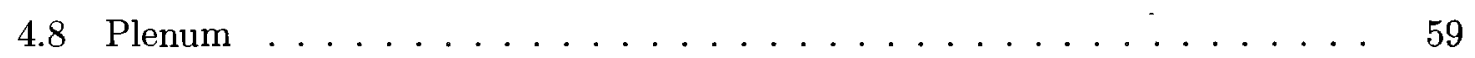

5 Computational Study

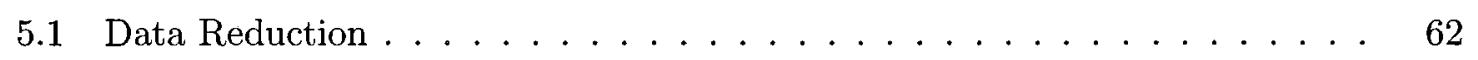

5.2 Pressure-Velocity Coupling . . . . . . . . . . . . . . . 63

5.3 Advection Scheme $\ldots \ldots \ldots \ldots \ldots \ldots$

5.4 Turbulence Modelling . . . . . . . . . . . . . . . . . 64

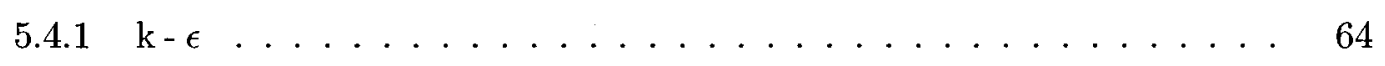

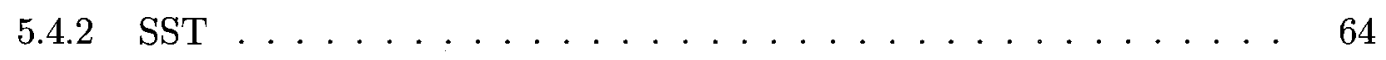


5.5 Computational Domain . . . . . . . . . . . . . . 65

5.5 .1 Boundary Conditions .................... 66

5.5 .2 Grid Structure . . . . . . . . . . . . . . . 68

5.5 .3 Grid Study . . . . . . . . . . . . . . . . 72

6 CFD-Based Parametric Study $\quad 78$

6.1 Effect of $\operatorname{Swirl} \ldots \ldots \ldots \ldots \ldots \ldots \ldots$

6.2 Effect of Stub Direction . . . . . . . . . . . . . . 85

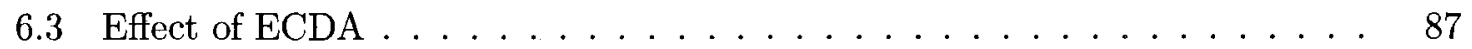

6.4 Effect of Wedge Angle . . . . . . . . . . . . . . . . . 89

6.5 Effect of Aspect Ratio and Area Ratio in the Annular to Rectangular Transition Region . . . . . . . . . . . . . . . . . . 92

6.6 Effect of Inlet Boundary Layer Blockage . . . . . . . . . . . . . 96

7 Correlation of the Total to Total Pressure Loss 99

7.1 Japikse Correlation of Annular Diffusers . . . . . . . . . . . . . . 100

7.2 Correlation of the CFD Results . . . . . . . . . . . . . . . . 104

8 Conclusion and Recommendation $\quad 111$

8.1 Conclusion . . . . . . . . . . . . . . . . . . . 111

8.2 Recommendation . . . . . . . . . . . . . . . . . . 112

$\begin{array}{lr}\text { Bibliography } & 114\end{array}$ 


\section{List of Figures}

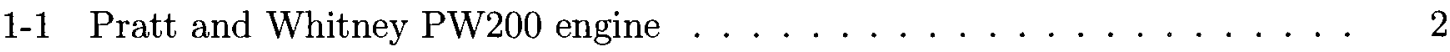

1-2 Pratt and Whitney PT6 engine .................. 2

1-3 Exhaust duct geometric features ................ 3

2-1 Conical diffuser presented with dimensional parameters $\ldots \ldots . \ldots$

2-2 Conical diffuser performance chart based on data from Cockrell and Markland $\left(B_{1} \approx 20\right) \ldots \ldots \ldots \ldots \ldots \ldots \ldots \ldots \ldots \ldots \ldots \ldots \ldots \ldots \ldots \ldots \ldots \ldots$

2-3 Conical diffuser performance chart from McDonald and Fox . . . . . . . . . 10

2-4 Diffuser performance coefficient as a function of area ratio for (a) axial inlet flow (b) swirling inlet flow . . . . . . . . . . . . . . . . 11

2-5 Radial distributions of total and static pressures at the inlet section . . . . 12

2-6. Variation of total pressure along stream surfaces of revolution . . . . . . . 12

2-7 Maximum Pressure Recovery of Conical and Square Diffusers $-M_{t h}=0.8 \quad . \quad 13$

2-8 Variation of the total pressure loss coefficient with entrance length (X/D) for a $5^{\circ}$; a) Reynolds number $1 \times 10^{5}$, b) Reynolds number $4 \times 10^{5} \ldots 13$

2-9 Conical diffuser loss map using Sharan's data . . . . . . . . . . . . . . . . 14

2-10 Exit discharge area ratio for conical diffusers on $C p^{*}$ (based on data from Cockrell and Markland) . . . . . . . . . . . . . 15

2-11 Annular diffusers (a) equiangular (b) straight core (c) double divergent . . . 16

2-12 Annular diffuser performance chart $\left(B_{1} \approx .02\right) \ldots \ldots \ldots$

$2-13$ Pressure recovery contours . . . . . . . . . . . . . . . 17

2-14 Straight annular diffuser performance with swirl $\ldots \ldots \ldots \ldots$

$2-15$ Performance of equiangular diffusers . . . . . . . . . . . 18 
2-16 Loss map using Stevens and Williams data . . . . . . . . . . .

2-17 Variation of the total pressure loss coefficient with entry blockage (Figure adapted from Klein) . . . . . . . . . . . . . . 20

2-18 Exit discharge area ratio for annular diffusers on $C p^{*} \ldots \ldots \ldots$

$2-19$ Experimental setup . . . . . . . . . . . . . . . . . . 22

2-20 2D Cross-sectional View of Exhaust . . . . . . . . . . . . . 22

$2-21$ Schematic of experimental setup f . . . . . . . . . . . 25

$2-22$ Domain of CFD grid used for computations . . . . . . . . . . . 26

3-1 Equivalent Cone Diffusion Angle Levels Studied . . . . . . . . . . . . . . 32

$3-2$ Duct Wedge Angle Levels Studied $\ldots \ldots \ldots$. . . . . . . . . . 33

3-3 Gas Path Aspect Ratio. . . . . . . . . . . . . . . . . . . . 34

3-4 Gas Path Aspect Ratio Levels Studied . . . . . . . . . . . . . . . . 35

3-5 Duct Locations where Area is Specified f . . . . . . . . . . . . 36

$3-6$ Exhaust Stub Direction $\ldots \ldots \ldots \ldots \ldots \ldots$

3-7 Sample of exhaust duct inlet swirl gradients . . . . . . . . . . . . . 39

$3-8$ Boundary layer axial velocity profiles . . . . . . . . . . . . . . . 41

4-1 Sample P\&WC Single Port Swept Exhaust Duct . . . . . . . . . . . 48

$4-2$ Equivalent Cone Diffusion Angle . . . . . . . . . . . . . . . . 49

$4-3$ Gas Path Conic . . . . . . . . . . . . . . . . . . . 50

4-4 Gas Path Spline and Aspect Ratio . . . . . . . . . . . . . . . 52

$4-5$ Flow Splitter . . . . . . . . . . . . . . . . 53

$4-6 \quad$ Flow Splitter Construction Plane . . . . . . . . . . . . . . . . 55

$4-7$ Flow Splitter Construction Plain . . . . . . . . . . . . . . . 56

$4-8$ Flow Splitter Wedge Angle . . . . . . . . . . . . . . . 57

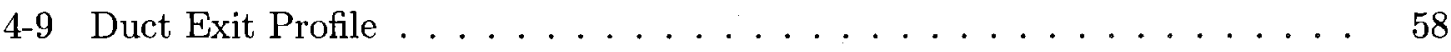

4-10 Duct Cross-Sections Downstream of Flow Splitter . . . . . . . . . . . . . 59

4-11 Duct Surface Passing Through Cross-Sections . . . . . . . . . . . . . 60

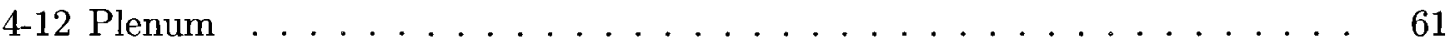

5-1 Air solid model of the computational domain . . . . . . . . . . . 65 
$5-2 \quad$ CFD boundary conditions $\ldots \ldots \ldots \ldots \ldots \ldots \ldots$

5-3 Exhaust Duct Inlet Showing Prism Layer Elements . . . . . . . . . . . 69

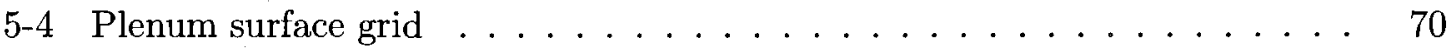

5-5 Cross-section of the plenum domain showing element sizes . . . . . . . 71

$5-6 \frac{\Delta P_{t t}}{q_{1}}$ ploted versus total number of prism layers $\ldots \ldots \ldots \ldots$

5-7 Three grids constructed from tetrahedral sizes of $h / 3$ (coarse), $h / 6$ (intermediate), and $h / 12$ (fine) . . . . . . . . . . . . . . 74

$5-8 \quad \frac{\Delta P_{t t}}{q_{1}}$ ploted versus total number of nodes $\ldots \ldots \ldots \ldots \ldots$

$5-9$ Mach contours for the three grid densities $\ldots \ldots \ldots \ldots$

6-1 Cross-sections used for data reduction and flow visualization . . . . . . 78

6-2 Total pressure loss coefficient evaluated at each section defined in figure 6-1 79

6-3 Velocity contours normal to cross-section (nominal $0^{0}$ swirl) . . . . . . . 80

6-3 Velocity contours normal to cross-section (nominal0 $0^{0}$ swirl)....con't . . . . 81

6-4 Velocity contours normal to cross-section (nominal $35^{0}$ swirl) . . . . . . . 82

6-4 Velocity contours normal to cross-section (nominal $35^{0}$ swirl)....con't . . . 83

6-5 Mach contours at mid plane demonstrating the effect of stub direction (nominal $0^{0}$ inlet swirl $\ldots \ldots \ldots \ldots \ldots \ldots$

6-6 Normalized static pressure contours at mid plane demonstrating the effect of stub direction (nominal $0^{0}$ inlet swirl) $\ldots \ldots \ldots$. . . . . . . 86

6-7 Total pressure losses calculated for C-shape, Straight, and S-shaped exhaust stubs (low inlet blockage) $\ldots \ldots \ldots \ldots$. . . . . . . . . . 87

6-8 Mach number contours at mid-plane demonstrating the effect of ECDA (inlet conditions: nominal $0^{0}$ swirl with low blockage) . . . . . . . . . . 88

6-9 Total pressure losses as a function of ECDA calculated at exit of the annulus 89

6-10 Wall shear stress contours (nominal $25^{0}$ swirl) . . . . . . . . . . . . 90

6-11 Wall shear stress contours (nominal $35^{0}$ swirl) . . . . . . . . . . . . 91

6-12 Total pressure losses as a function of wedge angle calculated at section $3 \ldots 92$

6-13 Mach number contour plot on a plane through the flow splitter . . . . . . 93

6-14 Normalized wall static pressure contours demonstrating the effect of aspect ratio and area ratio in the annular to rectangular transition region . . . . . 
6-15 Mach number contours demonstrating the effect of aspect ratio and area ratio in the annular to rectangular transition region . . . . . . . . . .

6-16 Mach number contours demonstrating the effect of inlet blockage on three exhaust ducts with ECDA of $0^{0}, 10^{0}$, and $20^{0} \ldots \ldots \ldots$

6-17 Total pressure loss coefficient vs. inlet swirl for low and medium inlet blockage 98

6-18 Total pressure loss coefficient vs. inlet swirl for medium and high inlet blockage 98

7-1 Diffuser effectiveness versus area ratio with low level inlet aerodynamic blockage and no inlet swirl . . . . . . . . . . . . . . . 100

7-2 Diffuser effectiveness with principle geometric effects removed including data at all levels of inlet aerodynamic blockage . . . . . . . . . . . . . . . . 101

7-3 Diffuser effectiveness with the principle effects of geometry and inlet swirl removed according to preceding correlations . . . . . . . . . . . . 102

7-4 Diffuser effectiveness with the principle effects of geometry, inlet swirl, and inlet blockage removed . . . . . . . . . . . . . . . . 103

7-5 Total pressure loss trend in the exhaust for low inlet blockage . . . . . . . . 104

7-6 Total pressure loss trend in the exhaust for medium inlet blockage . . . . . 105

7-7 Total pressure loss trend in the exhaust for large inlet blockage . . . . . . 105

7-8 Surface passing through normalized losses for ECDA and aspect ratio (Solid circles are points lying above the surface and hollow circles are points lying

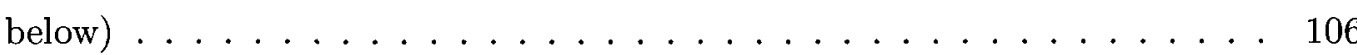

7-9. Normalized losses versus wedge angle with the effects of blockage, swirl, ECDA, and aspect ratio removed . . . . . . . . . . . . . 107

7-10 Normalized losses versus area ratio with the effects of blockage, swirl, ECDA, aspect ratio, and wedge angle removed . . . . . . . . . . . . 108

7-11 Plot of CFD losses vs. predicted losses between inlet and section-12 $\ldots 109$ 


\section{List of Tables}

2.1 Summary of performance of CFD analysis in the study of single port gas

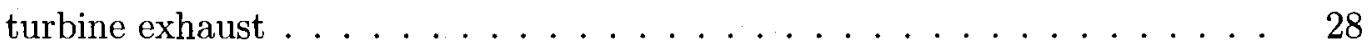

3.1 Exhaust Duct Area Ratio (referenced to figure 3-5) . . . . . . . . . . 37

3.2 Geometric Parameters . . . . . . . . . . . . . . . . . 42

3.3 Aerodynamic Parameters . . . . . . . . . . . . . . . 42

$3.4 \quad L_{9}$ Orthogonal Array $\ldots \ldots \ldots \ldots \ldots \ldots$

$3.5 \quad L_{9}$ Orthogonal Array Expanded for Stub Direction . . . . . . . . . . . . 46

7.1 Break Down of Correlation Accuracy . . . . . . . . . . . . . . . . 108 


\section{Nomenclature}

Variables

A

$A_{B}$

$A_{E}$

$A R$

$A S$

$C_{p}$

$C_{p i}$

D

$D_{h}$

E

$g$

$h$

$H$

$k$

$K$

$L$

M

$P$

$P t$

$q$

$r$

$T$

$T t$

$u$

$u_{\theta}$

$U$

area $\left(i n^{2}\right)$

blockage area $\int_{A}\left(1-\frac{u}{U}\right) d A$

effective area $\int_{A} \frac{u}{U} d A$

area ratio

aspect ratio

static pressure recovery coefficient $\frac{\overline{P_{2}}-\overline{P_{1}}}{\overline{q_{1}}}$

ideal pressure recovery coefficient $1-\frac{1}{A R^{2}}$

diameter (in)

hydraulic diameter $($ in $)=\frac{4 \cdot \text { cross-sectional area }}{\text { wetted perimeter }}$

effective area ratio $\frac{A_{E}}{A}$

swirl parameter $\frac{\int_{0}^{R}\left(u u_{\Theta} r^{2}\right) d r}{R \int_{0}^{R}\left(u^{2}\right) r d r}$

annulus height (in) $=r_{o}-r_{i}$

height (in)

turbulent kinetic energy

total pressure loss coefficient $\frac{\overline{P t_{2}}-\overline{P t_{1}}}{\overline{q_{1}}}$

diffuser length (in)

Mach number

static pressure (psi)

total pressure (psi)

dynamic pressure (psi)

radius (in)

static temperature $(\mathrm{R})$

total temperature $(\mathrm{R})$

axial velocity $(\mathrm{ft} / \mathrm{s})$

tangential velocity $(\mathrm{ft} / \mathrm{s}$ )

maximum axial velocity in cross section $(\mathrm{ft} / \mathrm{s}$ ) 
Variables

$\begin{array}{ll}\Delta P_{t t} & P_{t 1}-P_{t 2} \\ \Delta P_{t s} & P_{t 1}-P_{2}\end{array}$

Greek Symbols

$\begin{array}{ll}\alpha & \text { average swirl angle } \\ \beta & \text { blockage } \frac{\int_{A} \frac{u}{V} d A}{\int_{A} d A} \times 100 \\ \delta & \text { boundary layer thickness (in) } \\ \epsilon & \text { turbulence disipation rate } \\ \eta & \text { diffuser effectiveness } \frac{C_{p}}{C_{p_{i}}} \\ \theta & \text { half cone angle } \\ \rho & \left.\text { density (slug/in }{ }^{3}\right) \\ \omega & \text { turbulent frequency }\end{array}$

Subscripts

1

2

$i$

$o$

\section{Superscripts}

duct inlet

duct outlet

inner

outer

mass averaged quantity 


\section{Chapter 1}

\section{Introduction}

\section{$1.1 \quad$ Background}

Turboprop and Turboshaft gas turbine engines find their way in many aerospace and industrial applications. Figures 1-1 and 1-2 give a schematic layout of such gas turbines where each engine component is labelled. Careful review of these two figures demonstrates that the engine layout results in two very different exhaust ducts. In the first figure, Fig 1-1, an annular exhaust duct is found, where the flow and losses are rather well understood. In the second figure, Fig. 1-2, the engine layout does not allow for an annular exhaust duct resulting in the exhaust gases being redirected in a direction which differs from the inlet direction. A quick overview of the open literature shows that there is very little knowledge about these single port annular-to-rectangular exhaust ducts, be it flow physics, shape, design methods, performance, etc... Therefore, there is a need to study such exhaust ducts which redirect the combustion gases in a direction that differs from the inlet direction.

Significant gains in gas turbine performance can be made by reducing the exhaust duct loss. For example, consider a simple gas turbine cycle with a pressure ratio of 10 and at a constant power output, a $1 \%$ drop in the absolute back pressure to the turbine will result in a $1 \%$ improvement in the specific fuel consumption, therefore care should be taken to design an exhaust duct that would minimize any pressure loss. 


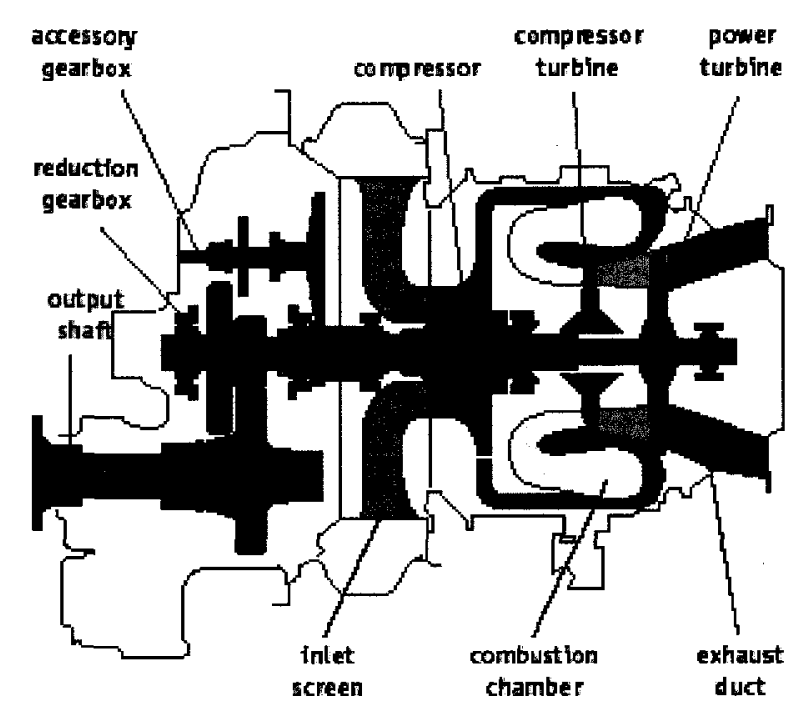

Figure 1-1: Pratt and Whitney PW200 engine (source: www.pwc.ca)

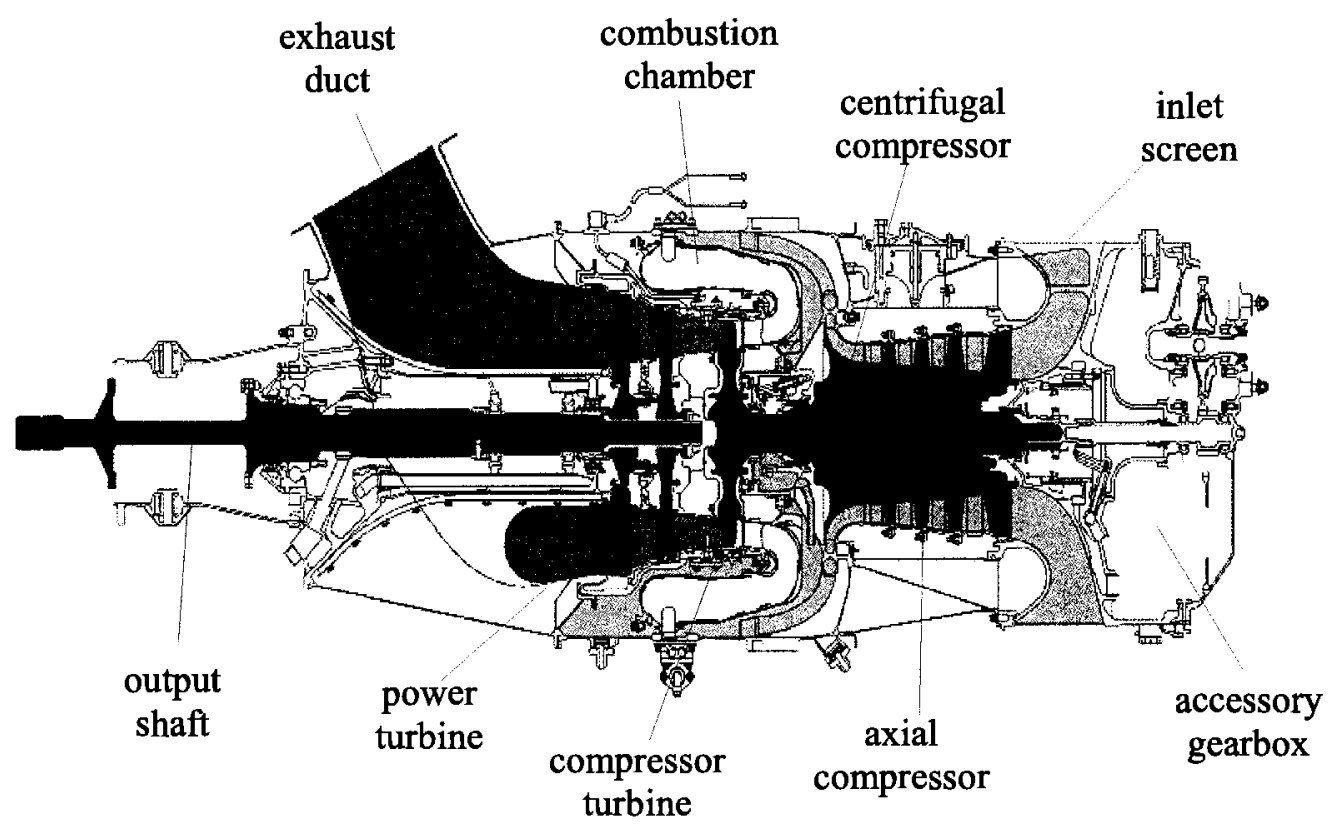

Figure 1-2: Pratt and Whitney PT6 engine (source: www.pwc.ca) 


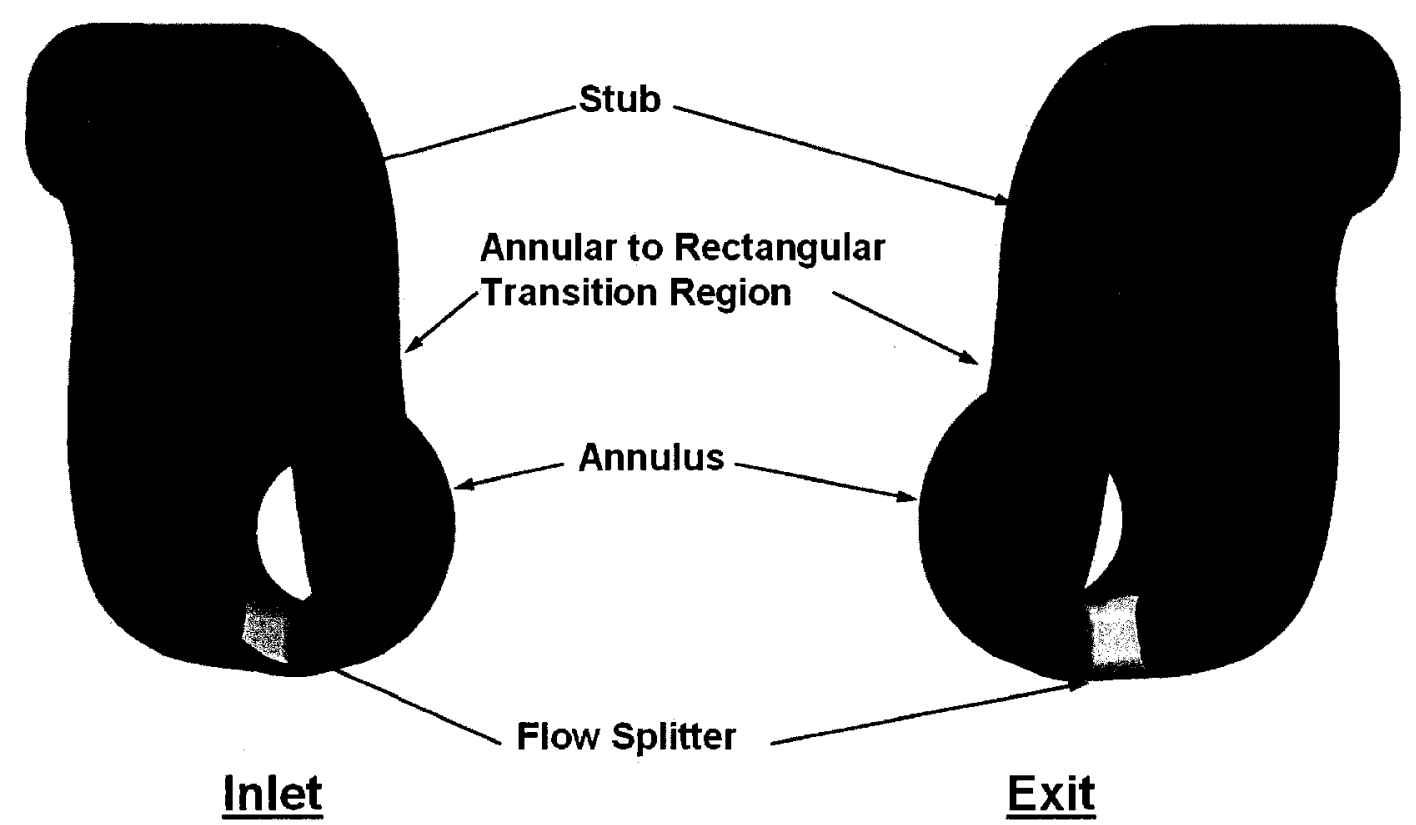

Figure 1-3: Exhaust duct geometric features

\subsection{Single Port Annular-to-Rectangular Exhaust Ducts}

As was explained in the previous section, single port annular-to-rectangular exhaust ducts find their use in gas turbine applications where the output shaft does not allow for an annular exhaust duct application. In this situation, the exhaust gases are required to be redirected, crossing over the output shaft, and diffused to ambient through a rectangular exhaust stub. The distinct geometric characteristics of these exhaust ducts are an annular inlet followed by a flow splitter, an annular to rectangular transition region, and a rectangular exhaust stub, see Fig. 1-3. The annular diffuser function is to efficiently diffuse the flow to a low Mach number before the gases enter the transitional region. Within the transitional region, the gases are forced to make an aggressive $90^{\circ}$ turn crossing over the power turbine shaft. It is in this region that the lower inlet Mach numbers will result in a lower loss making it important to obtain as much diffusion as possible in the upstream annular diffuser. A flow splitter is located at the start of the transition region to provide guidance to the gases directing the flow around the inner annulus surface. The exhaust gases enter a rectangular duct after 
crossing the inner annulus surface where diffusion is continued and the exhaust gases are directed to the ambient atmosphere. In aerospace applications, these exhaust ducts are bound to be small in size, making efficient diffusion difficult and sometimes impossible.

One such application of this exhaust duct configuration is found in PT6 engines produced by Pratt and Whitney Canada, Fig. 1-2. A PT6 engine has two spools, one for the gas generator, and a mechanically independent power turbine output shaft. The exhaust duct is mounted downstream of the last turbine stage. The flow into the exhaust duct is subsonic and swirling with the magnitude of swirl varying over the engine operating range.

\subsection{Contribution and Scope of the Present Study}

In this work, a pressure loss model is produced for single port annular-to-rectangular exhaust ducts. A parametric study was carried out using Computational Fluid Dynamics (CFD) to simulate the flow numerically in exhaust ducts where key geometric and aerodynamic parameters are varied and their effect on the total pressure loss is observed. Furthermore, the duct geometry is approximated through a five parameter model, which was coupled with a design of experiment method to generate a relatively small number of exhaust ducts for numerical simulation. The resulting numerical data that was produced, has been used as database to generate a total pressure loss model that designers can use as a tool to build more efficient non-symmetric gas turbine exhaust ducts.

The scope of the present study has been divided into the following sections consisting of:

\section{Theory and Literature Review}

- summary of past research on simple 2D conical and annular diffusers

- summary of past research on single port annular-to-rectangular exhaust ducts

2. Design of Experiment (DOE)

- n-dimension design space reduced to a five parameter model to approximate the duct geometry

- definition of the key inlet aerodynamic parameters 
- use methods of DOE to generate a relatively small number of exhaust ducts for numerical simulation

3. Geometry Synthesis

- synthesis of a single port annular-to-rectangular exhaust duct based on the five geometric parameters

4. Computational Study

- discussion on the use of a commercial CFD solver

- definition of the computational domain and grid structure

5. CFD-Based Parametric Study

- presentation and discussion of the results of the numerical study

6. Correlation of the Total to Total Pressure Loss

- review of a correlation produced by Japikse [7]

- correlation of the numerical data produced from the present work

7. Conclusion and Recommendation 


\section{Chapter 2}

\section{Theory and Literature Review}

\subsection{Diffuser Performance}

\subsubsection{Static Pressure Recovery Coefficient}

The static pressure recovery coefficient is defined as the static pressure rise across the diffuser divided by the inlet dynamic head:

$$
C p=\frac{\overline{P_{2}}-\overline{P_{1}}}{\overline{q_{1}}}
$$

For an incompressible and isentropic flow Bernoulli's equation can be used to define an ideal pressure recovery coefficient in terms the Area Ratio:

$$
C p_{i}=1-\frac{1}{A R^{2}}
$$

A useful expression for annular diffusers which relates the influence of inlet swirl $\alpha_{1}$ to the ideal pressure recovery is given by:

$$
C p_{i}=1-\frac{\bar{r}_{1}}{\bar{r}_{2}} \frac{\tan ^{2} \alpha_{1}+\left(h_{1}^{2} / h_{2}^{2}\right)}{\tan ^{2} \alpha_{1}+1}
$$




\subsubsection{Diffuser Effectiveness}

A useful parameter for evaluating the performance of a diffuser is through the diffuser effectiveness:

$$
\eta=\frac{C p}{C p_{i}}
$$

This relation relates the actual diffuser pressure recovery to the maximum potential pressure recovery.

\subsubsection{Total Pressure Loss Coefficient}

The diffuser total pressure loss coefficient, which is the parameter focused on in this work, is determined in the same manner as the static pressure recovery coefficient where the total pressure difference between diffuser inlet and outlet is divided by the inlet dynamic head:

$$
K=\frac{\overline{P t_{2}}-\overline{P t_{1}}}{\overline{q_{1}}}
$$

The total pressure loss coefficient for simple diffusers with uniform inlet and exit flow conditions can be determined from $\mathrm{Cp}$ and $C p_{i}$ through:

$$
K=C p_{i}-C p
$$

\subsection{Conical Diffusers}

\subsubsection{Geometry}

Conical diffusers, Fig. 2-1 are commonly characterized by various dimensionless parameters. Two of such dimensionless parameters are the dimensionless length, $L / D_{1}$, and area ratio, $A R=A_{2} / A_{1}$. The AR of a conical diffuser can further be described through the following geometric relation:

$$
A R=\left[1+2\left(L / D_{1}\right) \tan \Theta\right]^{2}
$$

Various studies have been performed on conical diffusers that take account inlet conditions as well as non-dimensional length and area ratio. One such study was performed by 


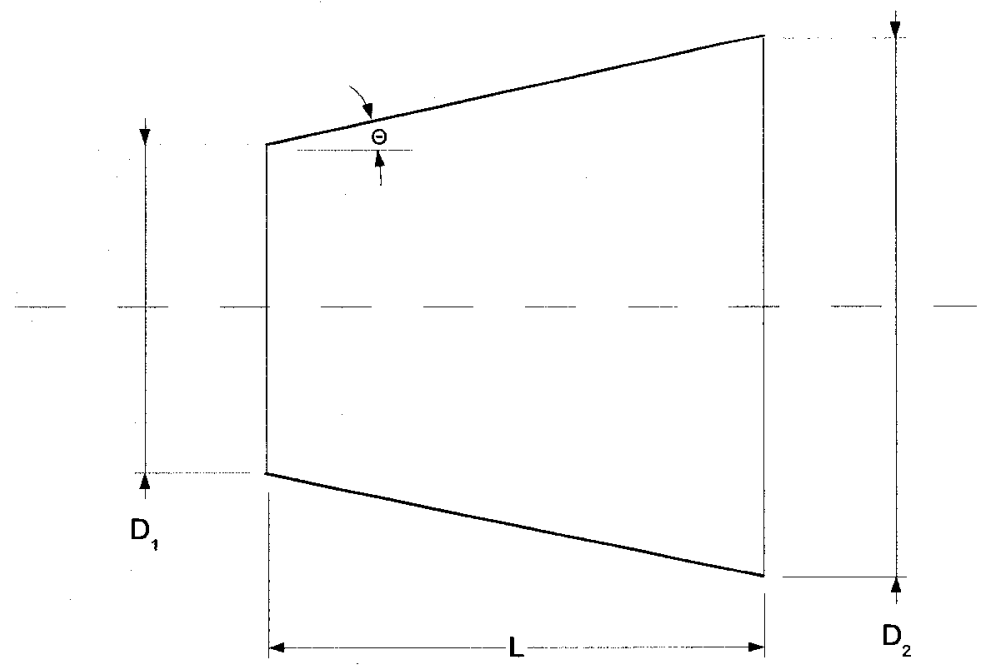

Figure 2-1: Conical diffuser presented with dimensional parameters

Cockrell and Markland and was presented as a performance chart, see Fig. 2-2, in a paper written by Sovran and Klomp [1]. This chart presents pressure recovery coefficient $(C p)$ versus non-dimensional length and area ratio. Furthermore the locus of the maximum pressure recovery for both non-dimensional length $\left(C p^{*}\right)$ and area ratio $\left(C p^{* *}\right)$ can be found. Another study performed by McDonald and Fox [2] has produced a performance map with similar results, Fig. 2-3.

\subsubsection{Swirl}

The performance of a conical diffuser can be affected significantly when a swirling component is introduced to the flow field. The effects of swirl is different depending on the type of swirl distribution (free vortex, forced vortex) effecting both the boundary layer and the core of the flow. The importance of swirl on diffuser performance can be seen in the work of McDonald et al. [9]. It is evident in Fig. 2-4 that the introduction of swirl can result in a conical diffuser approaching the theoretical ideal performance.

Additional studies were performed by Senoo et al. [10], who studied conical diffuser performance with swirling flow inlet conditions. The magnitude of swirl studied is quantified 


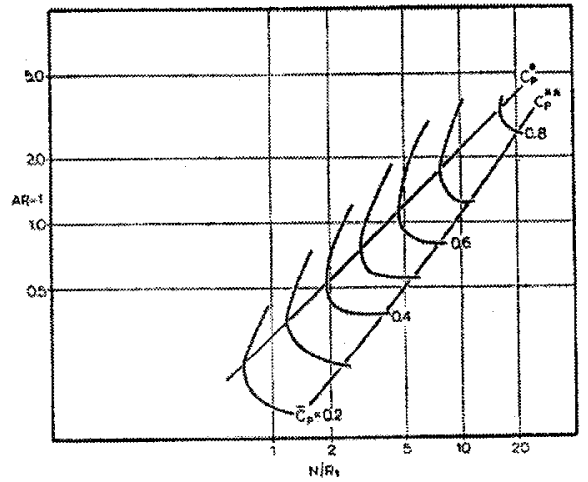

Figure 2-2: Conical diffuser performance chart based on data from Cockrell and Markland $\left(B_{1} \approx .20\right)[1]$

through a swirl parameter defined as:

$$
g=\frac{\int_{0}^{R}\left(u u_{\Theta} r^{2}\right) d r}{R \int_{0}^{R}\left(u^{2}\right) r d r}
$$

These authors identified the development of a Rankin vortex type flow composed of a large solid vortex core at the center of the conical diffuser where the axial velocity was very low. The total and static pressure distribution for an inlet swirl parameter of $\mathrm{g}=0.18$ is shown in Fig. 2-5. In Fig. 2-6, Senoo et al. have presented stream surfaces of revolution starting from the axis to $100 \%$ at the wall. Total pressure is observed to rise in the core of the flow up to a stream surface at $10 \%$ and again for $80 \%$ and $90 \%$. This is due to the low momentum flow at the core and the wall being dragged along by the main flow, and inversely, the low momentum flow slows down the main flow producing a total pressure drop.

\subsubsection{Aerodynamic Blockage}

The effect of aerodynamic blockage in conical diffusers has been studied by many researchers. One study by Livesey and Odukwe [13] looked at the length of the pipe preceding the conical diffuser. Their results show that as the inlet pipe length is increased, the boundary layer thickness increases resulting in a reduction in pressure recovery. A clear presentation of the effect of aerodynamic blockage on pressure recovery was made by Dolan and Runstadler 


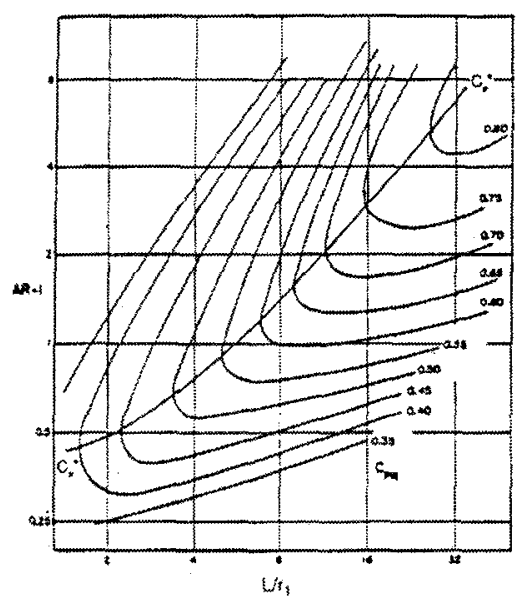

Figure 2-3: Conical diffuser performance chart from McDonald and Fox [2]

[11] shown in Fig. 2-7. This study made a comparison between a conical diffuser and a straight channel diffuser showing that the aerodynamic blockage is a significant aerodynamic parameter. Sharan [12] carried out a study with careful measurements of pressure recovery and total pressure loss on a $5^{\circ}$ conical diffuser where he varied inlet pipe length, Reynolds Number, and turbulence intensity. The results of his work, Fig. 2-8, demonstrate that the total pressure losses increase with developing inlet blockage but following losses can be seen to fall as a result the inlet flow field developing its own flow structure. Japikse [14] has produced a loss map, see Fig. 2-9, using the data from Sharan where the data loosely followed the trend of $K=C p_{i}-C p$ demonstrating that inlet factors such as inlet pipe length, Reynolds Number, and turbulence intensity must be considered. Kline [15] has compared the results of numerous studies showing that approximately the same reduction in pressure recovery for inlet aerodynamic blockage levels of approximately $14 \%$. An early correlation was produced by Sovran and Klomp [1] who have postulated that, for geometries that are dominated by pressure forces (as oposed to viscous forces) the exit discharge can be correlated with inlet blockage and area ratio. The results of their attempt to correlate the data of Cockrell and Markland for conical diffuser geometries laying on the $C p^{*}$ line is presented in Fig. 2-10 demonstrating that the data collapse reasonable well. 

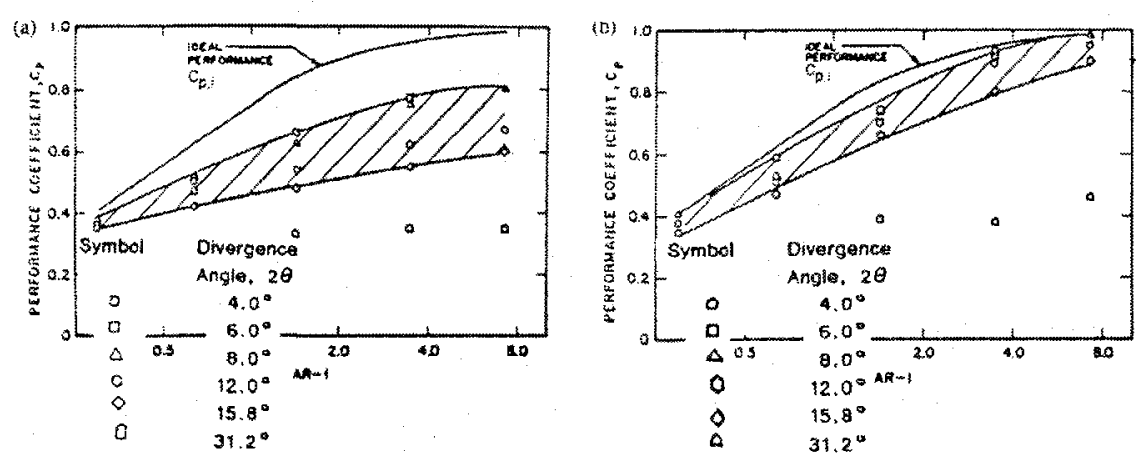

Figure 2-4: Diffuser performance coefficient as a function of area ratio for (a) axial inlet flow (b) swirling inlet flow [9].

\subsection{Annular Diffusers}

Annular diffusers are geometrically represented similar to conical diffusers; however more independent variables are present which require more complex relations. As in conical diffusers, non-dimensional length is represented as, $L / h$, and area ratio, $A R=A_{2} / A_{1}$. The AR of a three types of annular diffusers, shown in Fig. 2-11, can further be described through the following geometric relation where:

1. Equiangular case

$$
A R=1+2(L / h) \sin \Theta
$$

2. Straight Core is

$$
A R=1+\frac{2 L \sin \Theta}{h\left(1+r_{i} / r_{0}\right)}+\frac{L^{2} \sin ^{2} \Theta}{h^{2}}\left(\frac{1-r_{i} / r_{0}}{1+r_{i} / r_{0}}\right)
$$

3. Double divergent

$$
A R=1+2\left(\frac{L}{h}\right) \frac{\sin \Theta_{1}+\left(r_{i} / r_{o}\right) \sin \Theta_{2}}{1+r_{i} / r_{0}}+\left(\frac{L}{h}\right)^{2} \frac{\left[1-r_{i} / r_{0}\right]\left(\sin ^{2} \Theta_{1}-\sin ^{2} \Theta_{2}\right)}{1+r_{i} / r_{0}}
$$




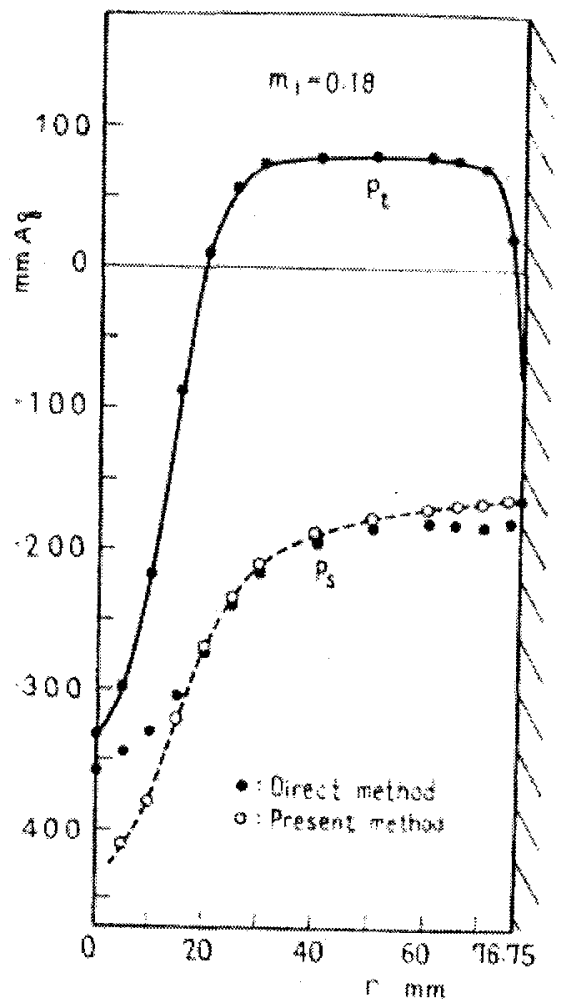

Figure 2-5: Radial distributions of total and static pressures at the inlet section [10]

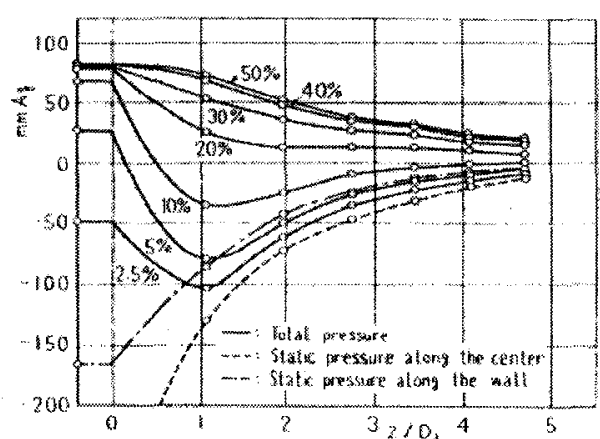

(a)

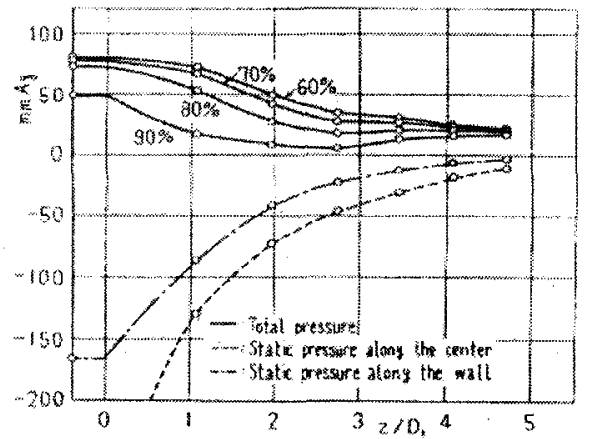

(b)

Figure 2-6: Variation of total pressure along stream surfaces of revolution [10] 


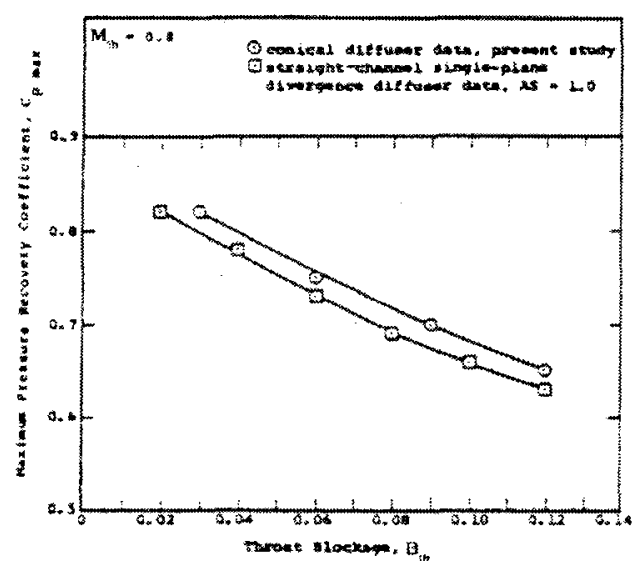

Figure 2-7: Maximum Pressure Recovery of Conical and Square Diffusers $-M_{t h}=0.8[11]$

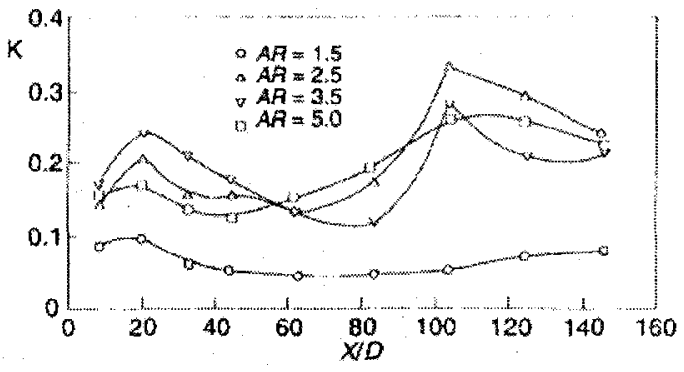

(a)

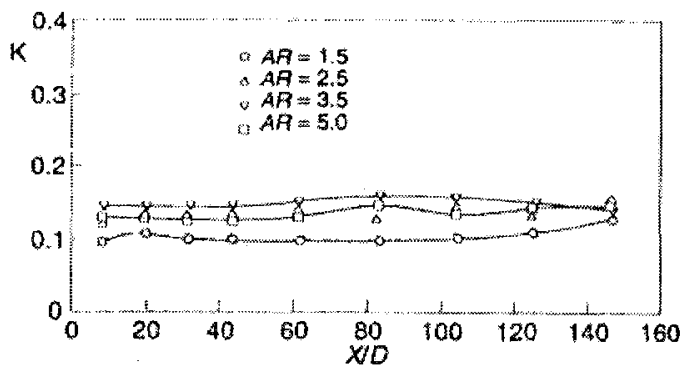

(b)

Figure 2-8: Variation of the total pressure loss coefficient with entrance length $(\mathrm{X} / \mathrm{D})$ for a $5^{\circ}$; a) Reynolds number $1 \times 10^{5}$, b) Reynolds number $4 \times 10^{5}[12]$ 


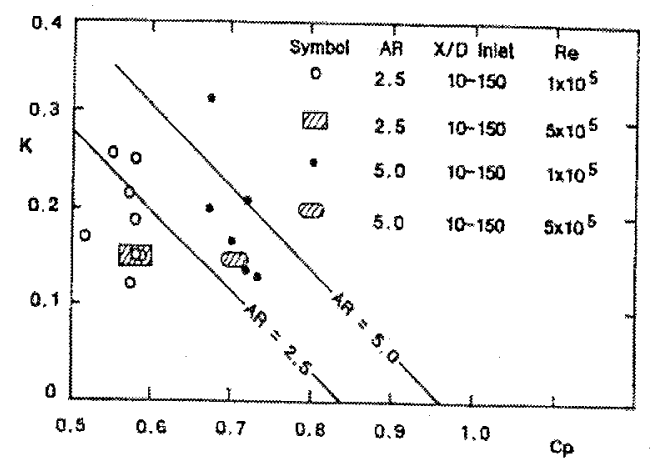

Figure 2-9: Conical diffuser loss map using Sharan's data [12]

Curved wall diffusers, which include axial to radial diffusers, are more complicated to describe requiring their own derivation of $L / h$ and AR specific to each shape.

Some of the first used annular diffuser maps produced by Sovran and Klomp [1] and Howard et al. [3] were published in 1967, Figs. 2-12 and 2-13. Their research examined an extensive selection of geometric diffuser types and produced detailed analysis of performance measurements. The diffuser map presented in Fig. 2-12 shows the bulk of configurations which gave the best performance. Same as with conical diffusers, the locus of the maximum pressure recovery for both non-dimensional length $\left(C p^{*}\right)$ and area ratio $\left(C p^{* *}\right)$ can be found. The main difference between these studies is that the research of Howard et al. covered fully developed inlet flow conditions while Sovran and Klomp covered low inlet blockage of approximately .02 .

\subsubsection{Swirl}

The effect of inlet swirl on pressure recovery has been studied by researchers and summarized by Japikse and Baines [4] in Fig. 2-14. For each of the diffusers tested, a common trend has been present. When inlet swirl is introduced the pressure recovery increases to a maximum in range of $10^{\circ}$ to $20^{\circ}$ inlet swirl, and then pressure recovery decreases thereafter. The effect of swirl on pressure recovery comes from two effects. The first is to press the flow against the outer annulus surface due to the centrifugal force delaying separation on this 


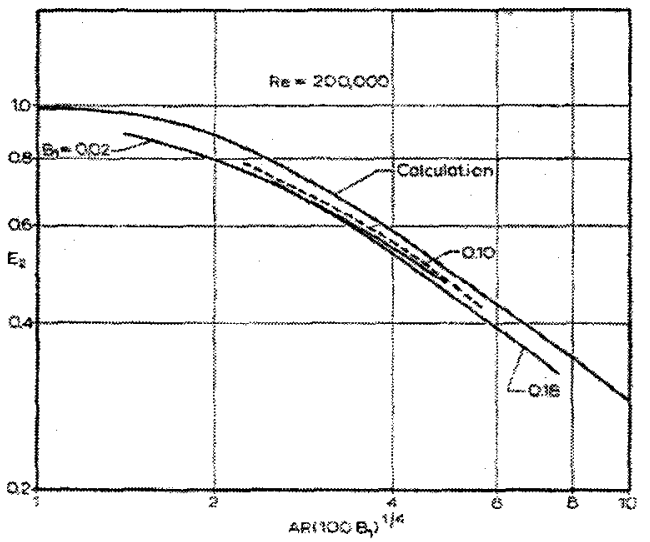

Figure 2-10: Exit discharge area ratio for conical diffusers on $C p^{*}$ (based on data from Cockrell and Markland)[1]

surface. The second comes from the centrifugal force destabilizing the inner hub boundary layer resulting in the boundary layer approaching flow separation at the hub surface. From the results shown in Fig. 2-14, it can be seen that the data of Coladipietro et al. shows that equiangular diffusers are more efficient than the others. Elkersh et al. [5] studied equiangular diffusers and confirmed the two effects that are produced are due to centrifugal forces acting on the outer and inner annulus boundary layers. Their results show improvement in pressure recovery up to inlet swirl values of $30^{\circ}$, and then decreasing performance with larger inlet swirl values, Fig. 2-15. It is also demonstrated in Fig. 2-15 that the total pressure losses tend to increase with increasing inlet swirl. A similar study to Elkersh et al. was performed by Dovzhik and Kartavenko [16] on equiangular diffusers confirming that the total pressure losses increase with increasing inlet swirl due to the intensity of flow separation at the outlet along the inner hub. Klomp [17] has tested eight annular diffuser families where the inner wall angles tested were both positive and negative. The results of this study demonstrated that all diffusers tested were relatively insensitive to free-vortex type swirl ranging from $0^{\circ}$ to $25^{\circ}$. Greater inlet swirl levels lead to hub separation which was not found to result in decreased performance in all diffusers tested. Swirl was found to have the largest impact on the diffuser families with negative inner wall angles. 


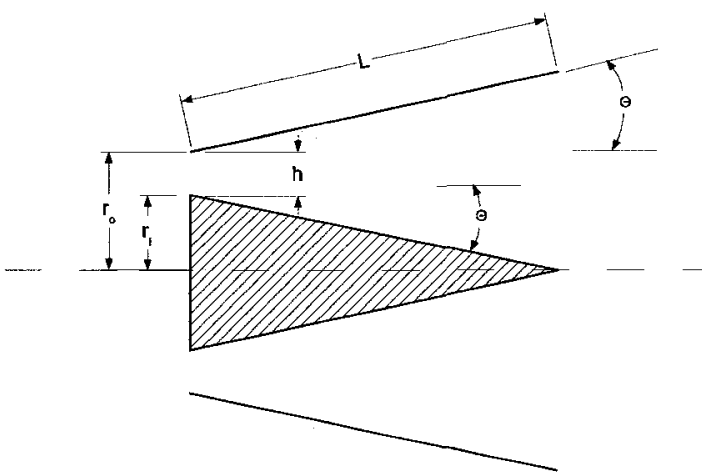

a)

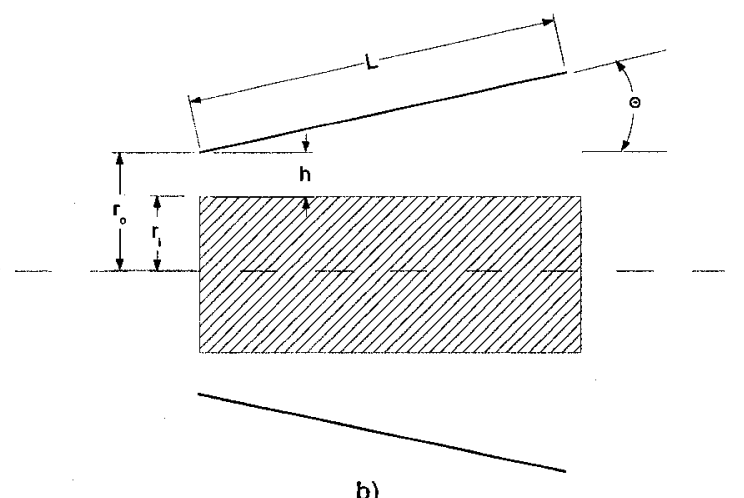

b)

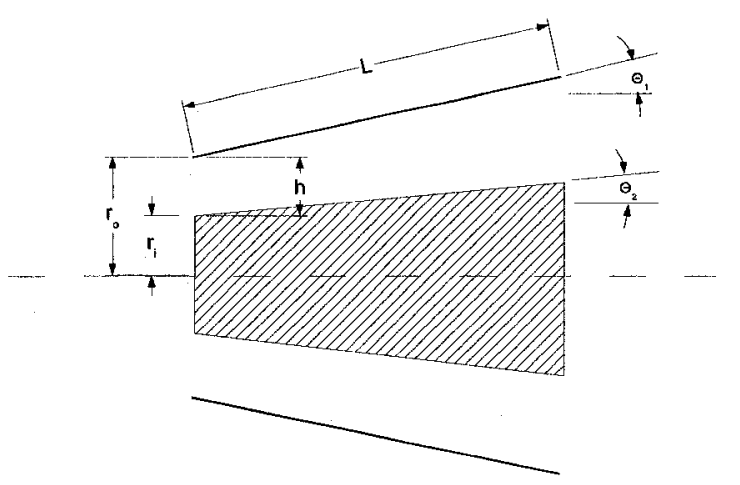

c)

Figure 2-11: Annular diffusers (a) equiangular (b) straight core (c) double divergent

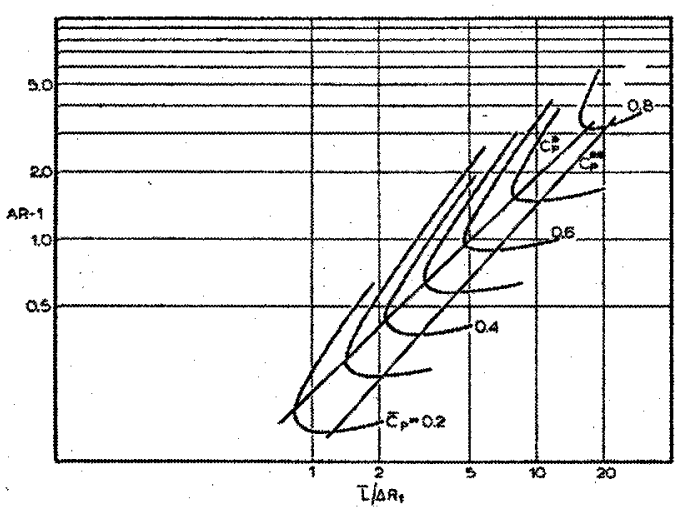

Figure 2-12: Annular diffuser performance chart $\left(B_{1} \approx .02\right)[1]$ 


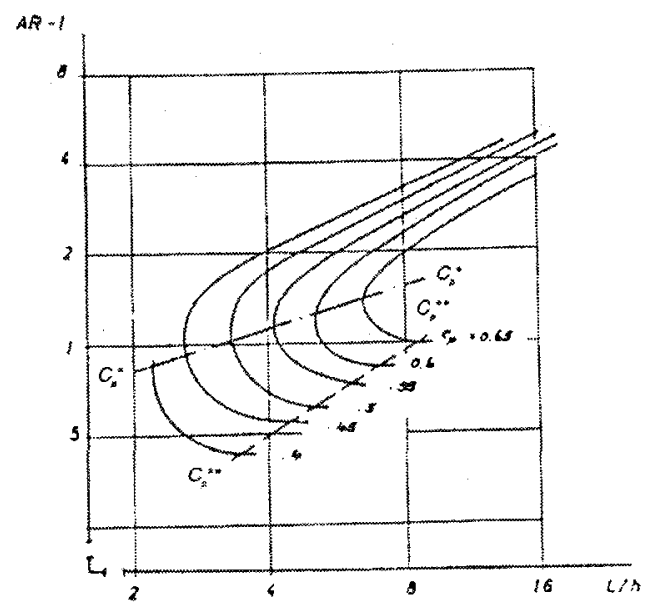

Figure 2-13: Pressure recovery contours [3]

\begin{tabular}{|c|c|c|c|c|c|c|c|c|}
\hline & ouse & as & $\begin{array}{l}q_{0} \\
\text { wow }\end{array}$ & bre & A4 & $r_{0}$ & 6 & 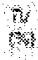 \\
\hline 0 & 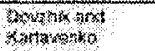 & 3 & 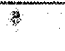 & 34 & 208 & ato & dits: & \\
\hline 5 & 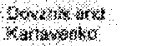 & e & 8 & As: & $2+0$ & 345 & fol & \\
\hline- & Maks & 80 & s & 79 & 31 & $\theta$ & $\begin{array}{l}\text { Wowa } \\
0 \% \text {. }\end{array}$ & \\
\hline 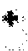 & Mrabiannat & 0 & 5 & 缯 & 67 & Q35: & 1, & \\
\hline 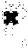 & Houdey aros & $\beta$ & 15 & 190 & 46 & 14 & $18 \%$ & \\
\hline 3 & Ooneowto of & 24 & $\infty$ & 126 & का & bats & 4 & 3 \\
\hline 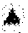 & 6067 somona & so & os & 206 & 3 & 68 & 60: & 8 \\
\hline$\geqslant$ & Cotrosowrow of & 21 & 20 & $\%$ & +2 & 00 & 36 & $\theta$ \\
\hline$\nabla$ & ostoopetsolo ol & 20 & $\beta$ & 3 & 180 & 083 & 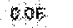 & $\mathrm{s}$ \\
\hline$\star$ & sham & 3 & 3 & 类. & 126 & 0,8 &,$+ y$ & \\
\hline
\end{tabular}

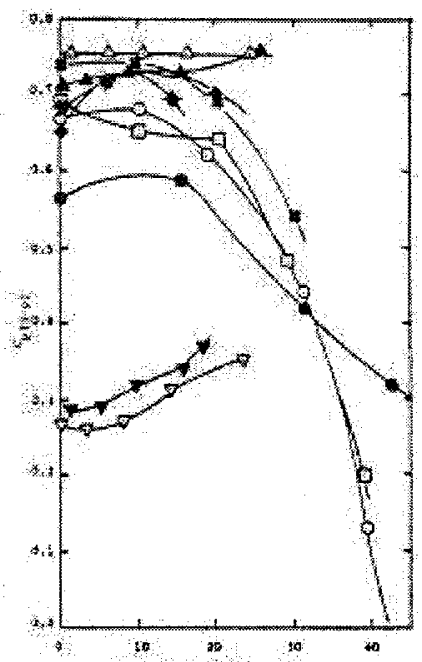

Figure 2-14: Straight annular diffuser performance with swirl [4] 


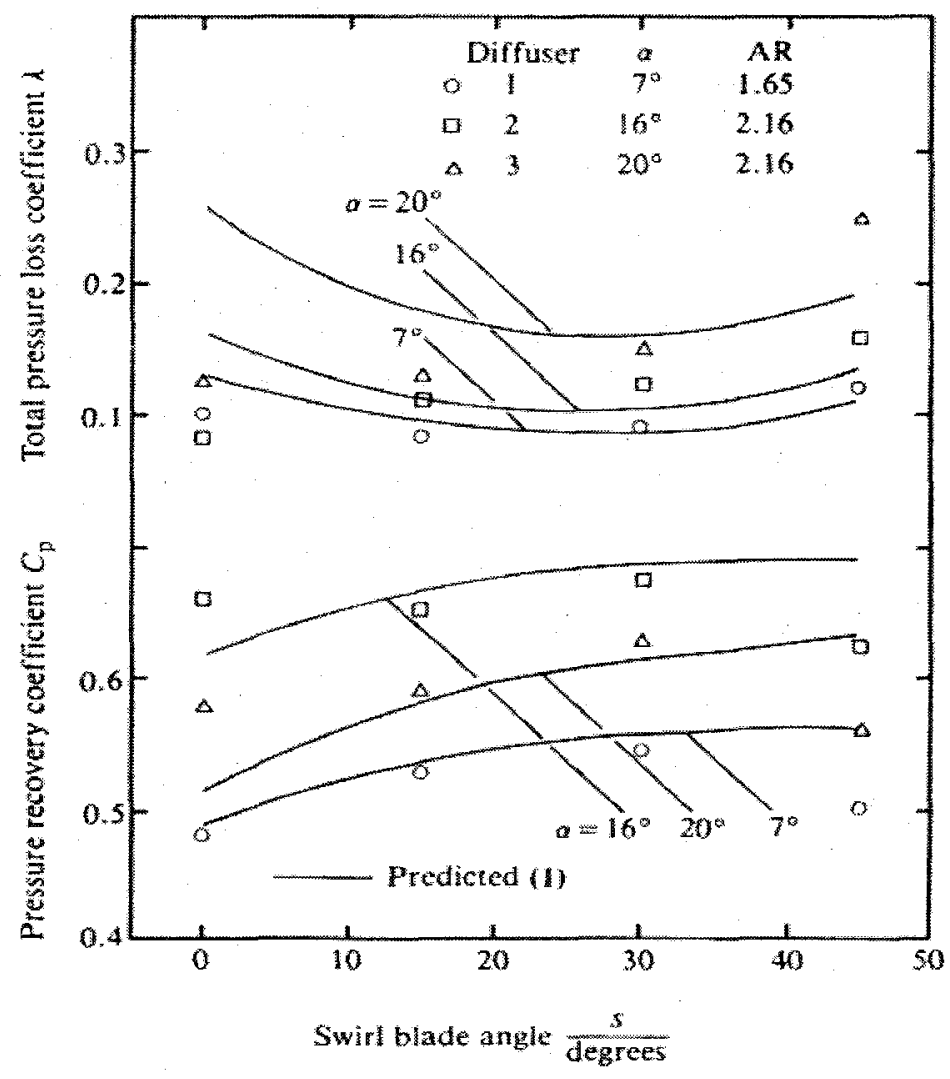

Figure 2-15: Performance of equiangular diffusers [5] 


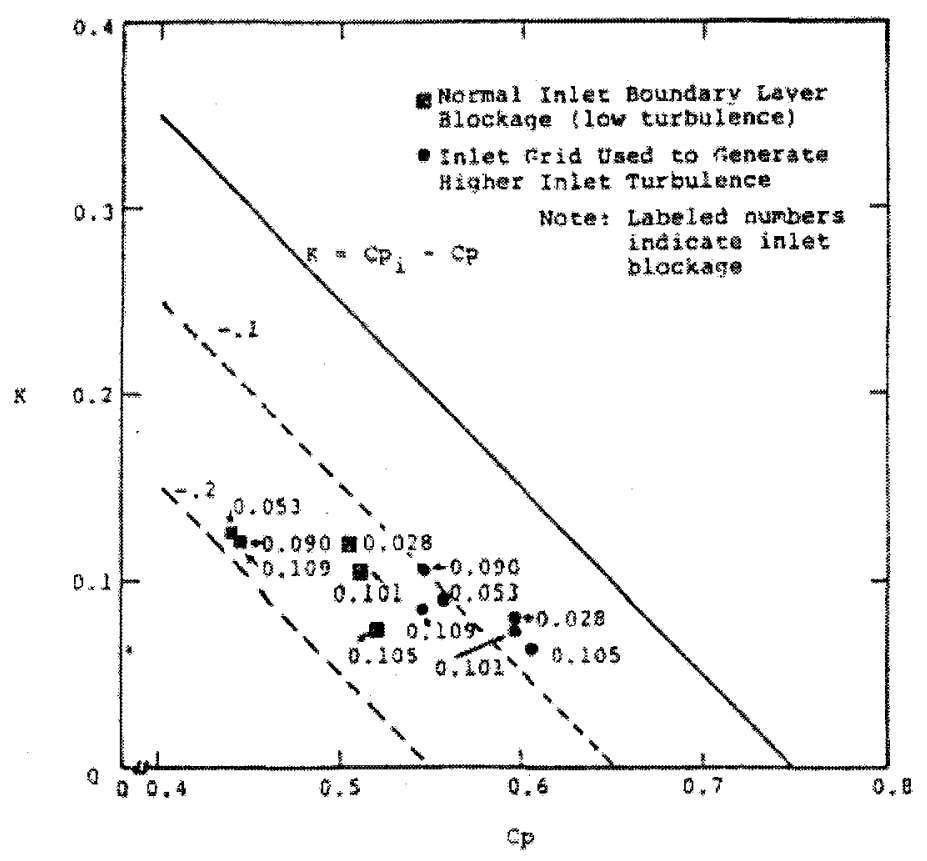

Figure 2-16: Loss map using Stevens and Williams data [4]

\subsubsection{Aerodynamic Blockage}

The effect of thick $(\beta=0.10)$ and thin $(\beta=0.06)$ inlet boundary layer blockage with swirl on annular diffuser performance was presented by Coladipietro et al. [18]. The authors comment on the discovery of a forced vortex for the condition of a thick inlet boundary layer partly due to the fact that the boundary layer penetrates deeply into the flow and meet near the center of the annulus [18]. For the condition of a thin boundary layer, a forced vortex is present near the wall but a free vortex is the predominant motion [18]. The authors discovered that $C p$ was higher in diffusers with small non-dimensional length with thin boundary layers and large non-dimensional length with thick boundary layers [18]. Japikse [4] presented numerous data published by Stevens and Williams [19] in a study showing the effect on inlet blockage, Fig. 2-16. From the data in this Fig. Japikse comments that increasing inlet blockage results in reducing diffuser pressure recovery, however, when long inlet lengths are present thus producing fully developed flow, the result is increasing pressure 


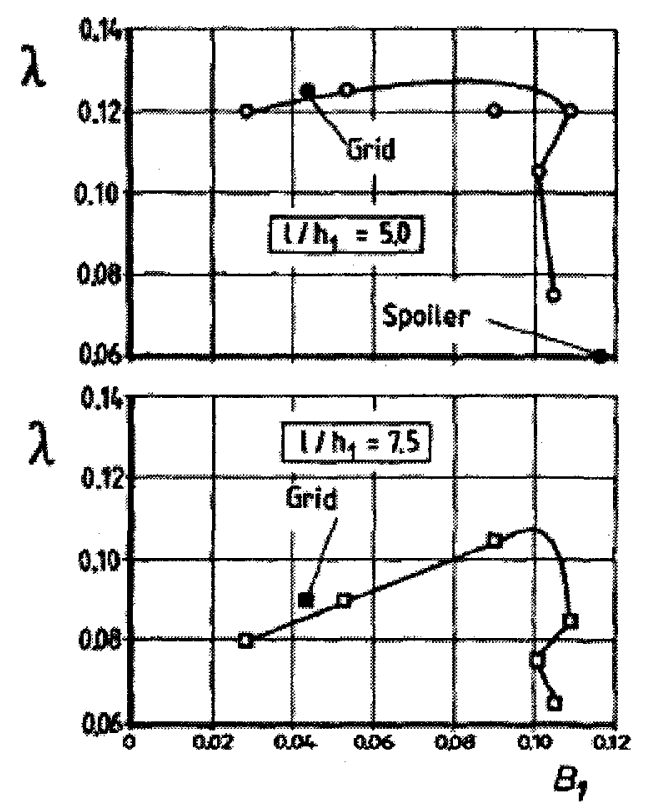

Figure 2-17: Variation of the total pressure loss coefficient with entry blockage (figure adapted from Klein [6])

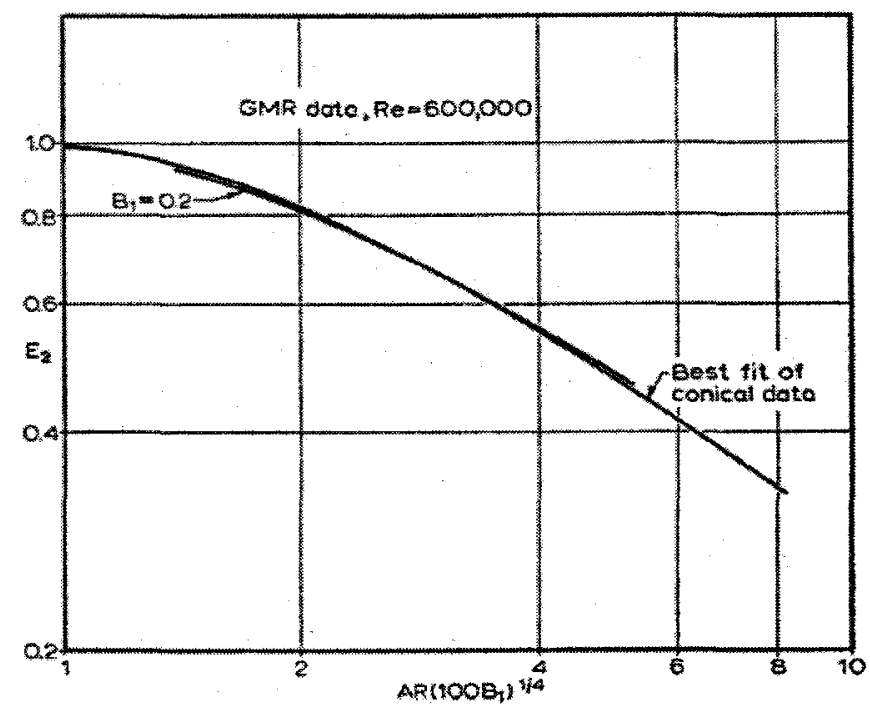

Figure 2-18: Exit discharge area ratio for annular diffusers on $C p^{*}[1]$ 
recovery. Klein [6] has taken the same data from Stevens and Williams and plotted it versus inlet blockage, Fig. 2-17, showing dramatic improvement in the total pressure losses with increasing inlet lengths. In the same manner as conical diffusers, Sovran and Klomp [1] have added their test data on annular diffusers to the correlation presented in Fig. 2-10 for conical diffusers, see Fig. 2-18, and found that for the inlet blockage tested the data are in agreement for both geometric types.

\subsection{Past Research Contributions}

\subsubsection{Loka et al.}

A numerical and experimental study was carried out at Pratt and Whitney Canada to achieve optimum integration of the PT6C-67A gas turbine engine on the Bell 609 aircraft [20]. The authors conducted the numerical analysis using an in-house finite element, compressible, Navier-Stokes CFD solver with a $k-\omega$ turbulence model. The efforts consisted of three phases; the first was to optimize the uninstalled engine; secondly the numerical simulation was expanded to include the installation effects which included the exhaust ejector system; lastly, experimental tests were conducted to validate the analysis.

\section{Experimental Study}

The experimental study was carried out on a full scale exhaust duct, Fig. 2-19. The exhaust duct was mounted to a blower which could not attain the normalized flow levels of an operating engine, therefore the authors had to extrapolate the data to represent exhaust performance for an engine in flight. Inlet conditions were produced through a swirl generator, which comprised of a series of adjustable vanes capable of producing swirl angles in the range of $0^{\circ}$ to $40^{\circ}$.

\section{Computational Study}

The computation domain consisted of a swept exhaust duct, an exhaust stub, and a plenum chamber, Fig. 2-20. The plenum chamber was created to capture the sudden expansion

of the exhaust gases into the atmosphere. The computational boundary conditions at the 


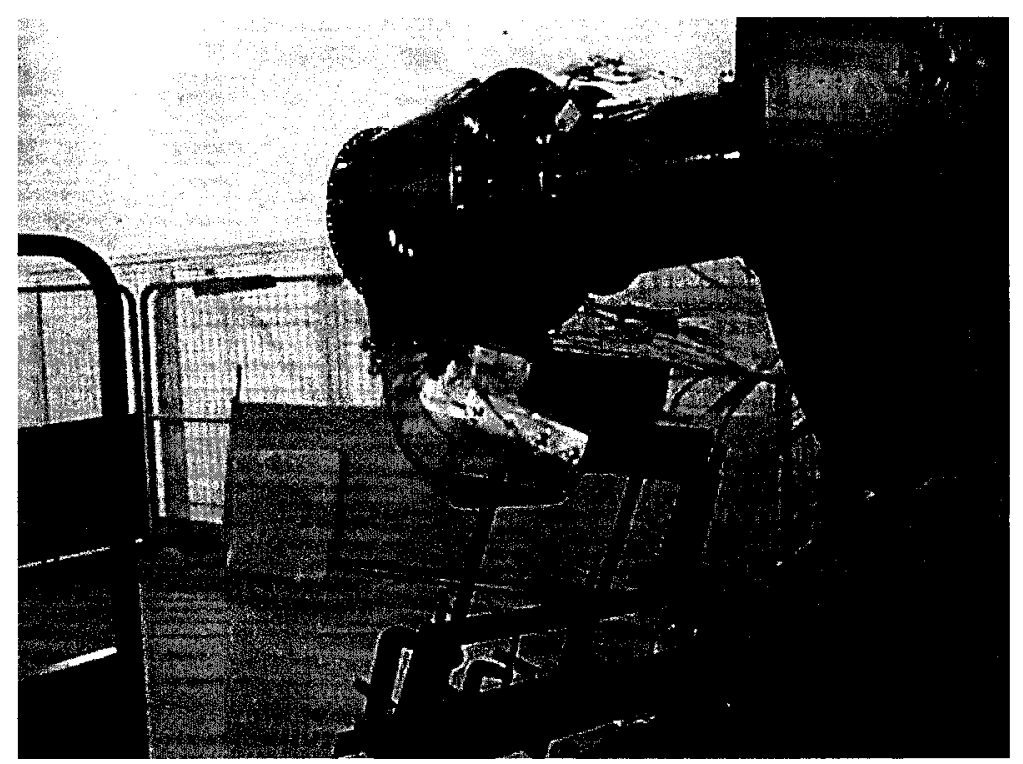

Figure 2-19: Experimental setup [20]

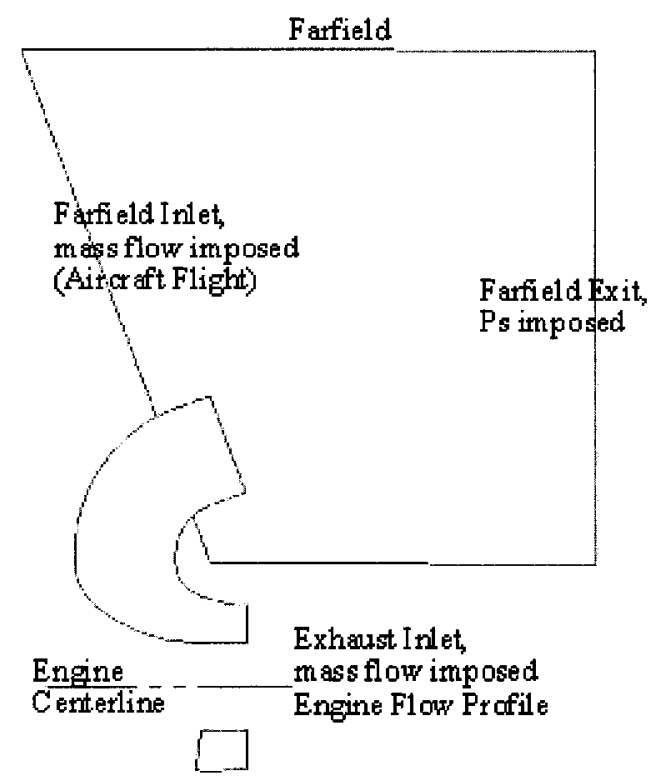

Figure 2-20: 2D Cross-sectional View of Exhaust [20] 
exhaust duct inlet were representative of engine profiles produced by the last stage turbine rotor blades. Far-field boundary conditions in the plenum were modeled corresponding to an aircraft in flight where, mass flow was imposed at the plenum inlet, and ambient static pressure at the plenum exit.

\section{Loss Mechanisms}

Based on the CFD results, the authors [20] suggest that there are three pressure loss mechanisms:

1. Incidence on the flow splitter: The authors have observed a stagnation zone on the suction surface of the flow splitter where the flow has separated. This stagnation zone results in narrow layer of separated flow along the hub surface which merges with hub wake.

2. A wake being shed from the hub surface due to the cross flow effects: The effect of the flow crossing over the hub toward the exit port leads to creating a wake downstream of the hub surface. The authors suggest the existence of a Von-Karmen vortex sheet and evidence of two counter rotating vortices.

3. Excessive diffusion along the inner curve resulting in flow separation: A peak in Mach number is found at the duct inner curve. The excessive diffusion in this region results in the flow separating producing a pressure loss

From the three loss mechanisms, two can be identified at the duct exit plane by regions of low total pressure; Hub separation and separation due to the inner curvature. It is suggested that the size of the low pressure regions dictate the magnitude of each loss mechanism. The authors have concluded that when there is no swirl at the turbine exit, the main loss mechanisms are due to the hub separation and the inner curvature which have been assessed to be equal contributors to the pressure losses. At higher swirl conditions, the incidence along the flow splitter will lead to larger losses which can not be identified at the duct exit because the separation merges with the hub wake. 


\section{Results and Conclusions}

A comparison was made between the CFD and experimental results where the total-to-total pressure loss coefficient, total-to-static pressure loss coefficient, and discharge coefficient are compared. The authors conclude that the trends predicted by the CFD are the same as what was found from rig testing, however the absolute levels varied between the two.

\subsubsection{Cunningham}

A detailed experimental and computational study was carried out on a single port tractor exhaust duct at Queens University in cooperation with P\&WC [8]. In this study, the objective was to determine the effect of inlet conditions and duct geometry on the flow structure and the level of overall pressure losses. Conclusions were also made on the suitability of boundary conditions for both experimental and computational work.

\section{Experimental Study}

The experimental study was carried out on a stereolithographic $\frac{1}{2}$ scale model of the tractor exhaust duct mounted to an annular cold flow wind tunnel, Fig. 2-21. A total of four geometries were studied experimentally. The wind tunnel used was capable of producing swirl, mass flow and inlet total pressure distributions similar to those seen in a gas turbine engine. The range of swirl studied consisted of zero swirl and two radial profiles provided by P\&WC which are representative of what a sample PT6 engine exhaust duct would see at the duct inlet plane. Total pressure profiling screens were used to produce circumferential non-uniform total pressure profiles at inlet to the duct.

\section{Computational Study}

Five geometries were studied computationally. The computational domain consisted of an inlet annulus, an exhaust duct, and a plenum chamber overlapping the exhaust duct exit, Fig. 2-22. The plenum is a large conical domain with boundary conditions to allow the exhaust jet to entrain flow freely into the plenum. The plenum also served to allow for a non-uniform pressure distribution at the exhaust duct exit plane which results from the large stream line curvature in the flow. 


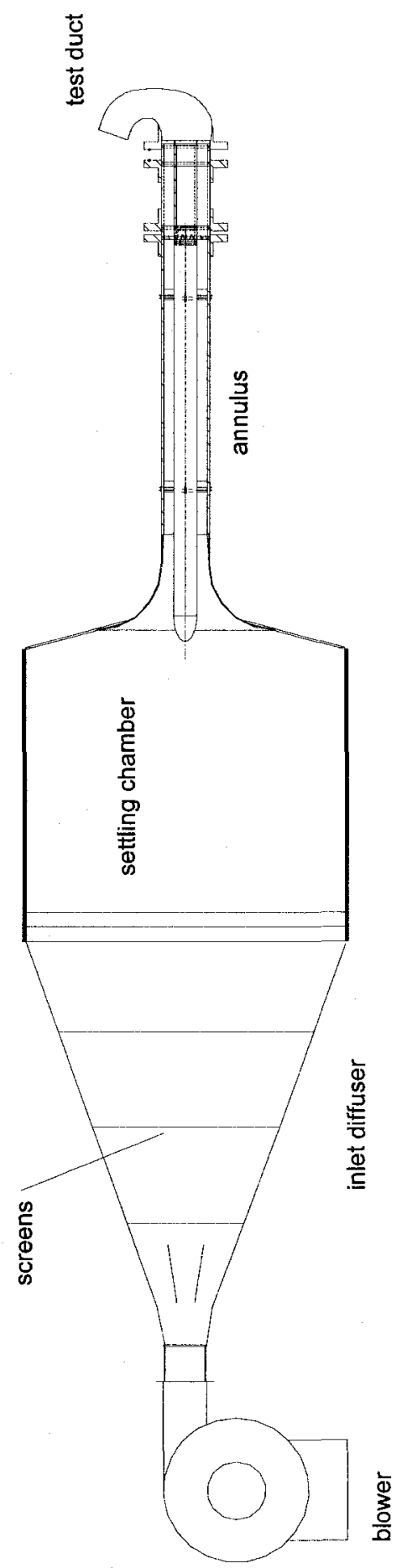

Figure 2-21: Schematic of experimental setup [8] 


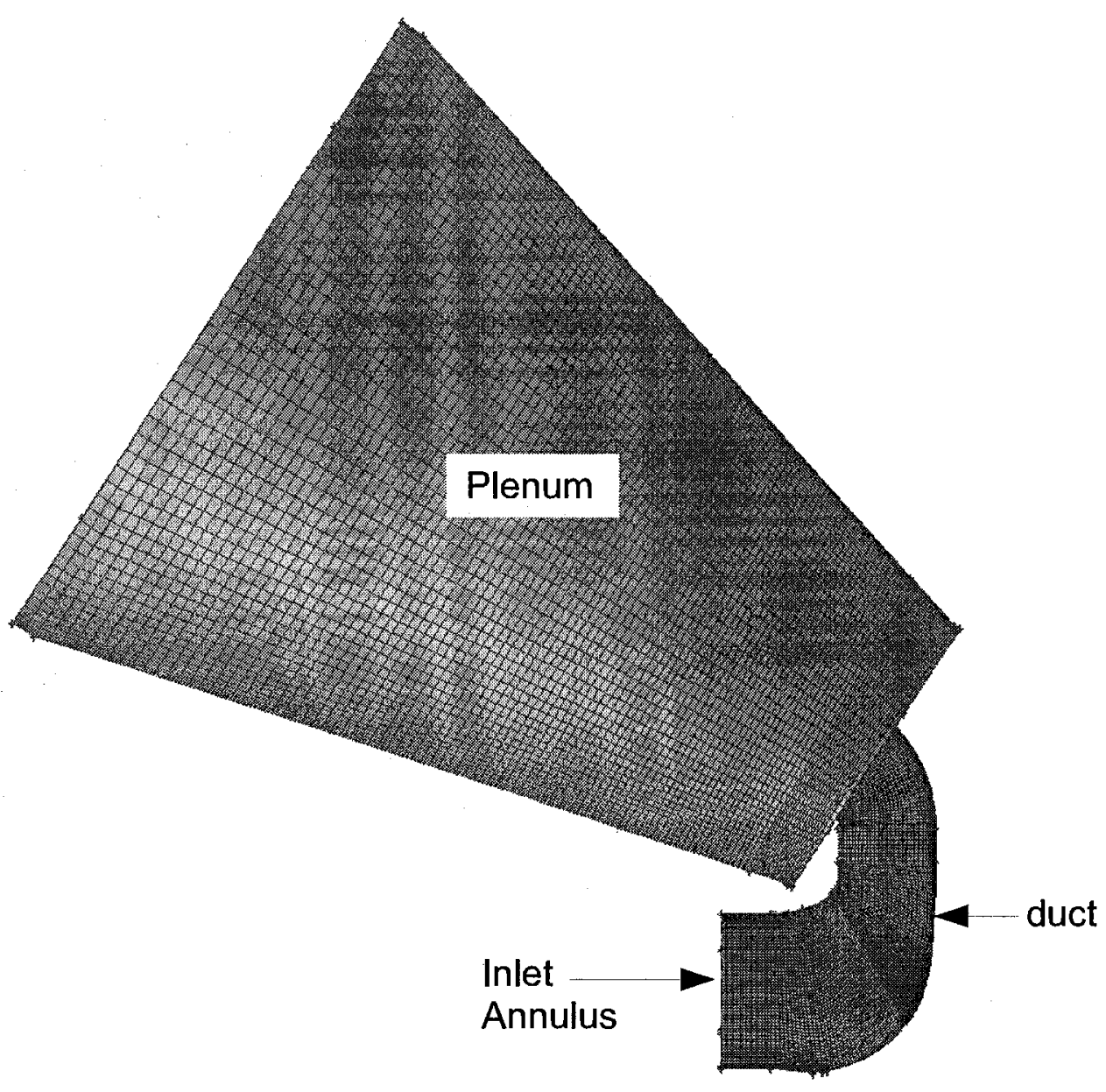

Figure 2-22: Domain of CFD grid used for computations [8] 
The computational grid was created using Gambit. Hexahedral elements were primarily used to produce a structured mesh with only the annular to rectangular transitional region requiring an unstructured grid composed of tetrahedral elements for ease of meshing. The boundary layer was defined with prism elements in the first rows of mesh from the duct surface. The flow solver used in this study was the commercial code Fluent 5.5. The most suitable turbulence model which was available was the RNG $k-\epsilon$ model; however the author used the realizable $k-\epsilon$ turbulence model through the majority of the study due to the difficulty in obtaining a converged solution using the former.

\section{Loss Mechanisms}

Cunningham [8] has identified three geometric parameters affecting the total pressure losses base on preliminary testing and literature:

1. Flow splitter.

2. Streamlining downstream of the center body.

3. Stub cross-sectional shape.

The three main pressure loss mechanisms were found and identified as:

1. Secondary flows: The secondary flows are generated through the duct bends as well as the presence of the flow splitter redirecting the flow across the center-body.

2. Flow non-uniformity: Present at the stub exit representing undiffused kinetic energy and therefore lower static pressure recovery. The exhaust stub cross-sectional shape influenced the exit effective area-ratio.

3. Flow separation and recirculation: The total pressure losses are a function of the flow separation and recirculation. Due to the complex shape of the exhaust duct, these losses dominated over skin friction losses. Flow separation occurs along the inside bend of the duct and in some cases, downstream of the center body. 


\section{Results and Conclusions}

Cunningham [8] made a comparison of experimental and CFD results concluding that the CFD consistently under-predicts the level of pressure losses in the exhaust ducts. CFD has on the other hand shown that it is capable of capturing trends in losses by accurately predicting changes in magnitude of pressure losses from one geometry to the next. When comparing pressure losses due to inlet swirl, Cunningham found that the slope of the trend line was under predicted when compared to measured results. This has been explained as the inability of the turbulence models to handle the anisotropy of highly swirling flows. Table 2.1 summarizes these findings.

Table 2.1: Summary of performance of CFD analysis in the study of single port gas turbine exhaust [8]

\begin{tabular}{|c|c|c|}
\hline Parameter & Suitability & Comments \\
\hline $\mathrm{E}$ & fair & Under-predicts distortion and secondary flow \\
\hline$\Delta \mathrm{Ptt}$ & fair & under-predicts losses \\
\hline$\Delta \mathrm{Pts}$ & fair & under-predicts losses \\
\hline geometry & good & $\begin{array}{l}\text { able to predict correct magnitude and trends } \\
\text { with change in geometry }\end{array}$ \\
\hline flow structure & fair & $\begin{array}{l}\text { easily gives details of internal flow structure, } \\
\text { may not be reliable in identifying separation }\end{array}$ \\
\hline efficiency & excellent & $\begin{array}{l}\text { very efficient for studying inlet conditions, } \\
\text { geometry limited by efficiency of mesher }\end{array}$ \\
\hline inlet conditions & excellent & inlet conditions can be easily specified \\
\hline
\end{tabular}

If CFD is to be used to design optimum exhaust ducts, Cunningham has made the following recommendations:

- In terms of predicting the total-to-static losses in the duct, the distribution of inlet flow has a large effect on the distribution of the outlet flow. To be able to make a realistic estimate of the total-to-static losses, a good estimate of the outlet flow is required. To ensure this, velocity boundary conditions should be applied at the inlet as this leads to a more realistic flow distribution at the exit. 
- A large plenum is required at the exit of the duct to produce accurate flow distortions in the duct near the exit.

- Turbulence models which account for swirl should be used if possible.

- Where possible, boundary conditions should be applied that account for the total pressure non-uniformities at the duct inlet resulting from the presence of the engine. 


\section{Chapter 3}

\section{Design of Experiment}

The work of Loka et al. [20], and Cunnigham [8] have identified many of the geometric and aerodynamic parameters responsible for the overall duct loss. In this chapter, more parameters are identified. Also discussed here are how the geometric parameters are quantified and bounded within a specific design space, giving limits to the magnitude of each parameter, for the purpose of creating a loss correlation. Next, combinations of each geometric parameter are grouped together to create a set of exhaust duct models which, later, will be simulated numerically along with the aerodynamic parameters to produce data for building a loss correlation.

\subsection{Geometric Design Space}

A design space can be envisioned as being an n-dimensional box which is capable of containing all practical exhaust duct shapes and sizes. The size of the box is chosen to allow each geometric parameter to be varied from a minimum to a maximum value which is thought to cover rather well the design space so that both good and bad performing exhaust ducts are represented. For each parameter, a minimum of three changes are required to be able to predict a non-linear trend with respect to exhaust duct losses. In this study, each geometric parameter will be extended to the minimum and maximum limits of the design space with one selection in the center. 


\subsubsection{Equivalent Cone Diffusion Angle}

The exhaust duct region between the inlet and the flow splitter can be represented as an annular duct. Quantifying an annulus using one parameter is done through an Equivalent Cone Diffuser Angle (ECDA). Equation 3.1 combines inlet annulus area, $A_{\text {in }}$, exit annulus area, $A_{\text {out }}$ and duct length, $L$, into one convenient parameter.

$$
E C D A=2 \arctan \left[\frac{\sqrt{\frac{A_{\text {out }}}{\pi}}-\sqrt{\frac{A_{\text {in }}}{\pi}}}{L}\right]
$$

The range of ECDA chosen for the axisymmetric annular duct is to provide for attached and separated flow. McDonald et al. [9] have found that optimal pressure recovery can be found for conical diffusers at cone angles between $6^{\circ}$ and $8^{\circ}$ with decreasing performance at larger cone angles. The work of Sovran and Klomp [1] has demonstrated that the optimum cone angle is $8^{\circ}$ provided that the area ratio is large between exit and inlet. It was therefore decided that an ECDA of $20^{\circ}$ should be sufficient to create flow separation given that downstream of the annular duct is the flow splitter and annular to rectangular transition region which could influence the streamwise pressure gradient and ensure that flow separation will occur. It is also of interest to see the effect of no diffusion before the annular to rectangular transition region where higher Mach numbers are expected to produce higher pressure losses in this region, therefore the minimum ECDA studied is $0^{\circ}$. An ECDA of $10^{\circ}$ is used as a middle value as it was found to closely approximate the sample P\&WC duct and is close to the optimal cone angles found by McDonald et al [9]. Figure 3-1 shows the annular ducts which were used in this study upstream of the annular to rectangular transition region.

\subsubsection{Flow Splitter Wedge Angle}

A swirling flow will create an incidence angle with the flow splitter leading edge possibly leading to form a separated region along the suction surface. It is expected that small wedge angles will lead to more severely separated flow resulting from larger incident angles. Using the sample duct provided by $\mathrm{P} \& W C$ as a the reference for a mid point value of around $45^{\circ}$, min and max values of $10^{\circ}$ and $80^{\circ}$ were chosen for this study, Fig. 3-2. 


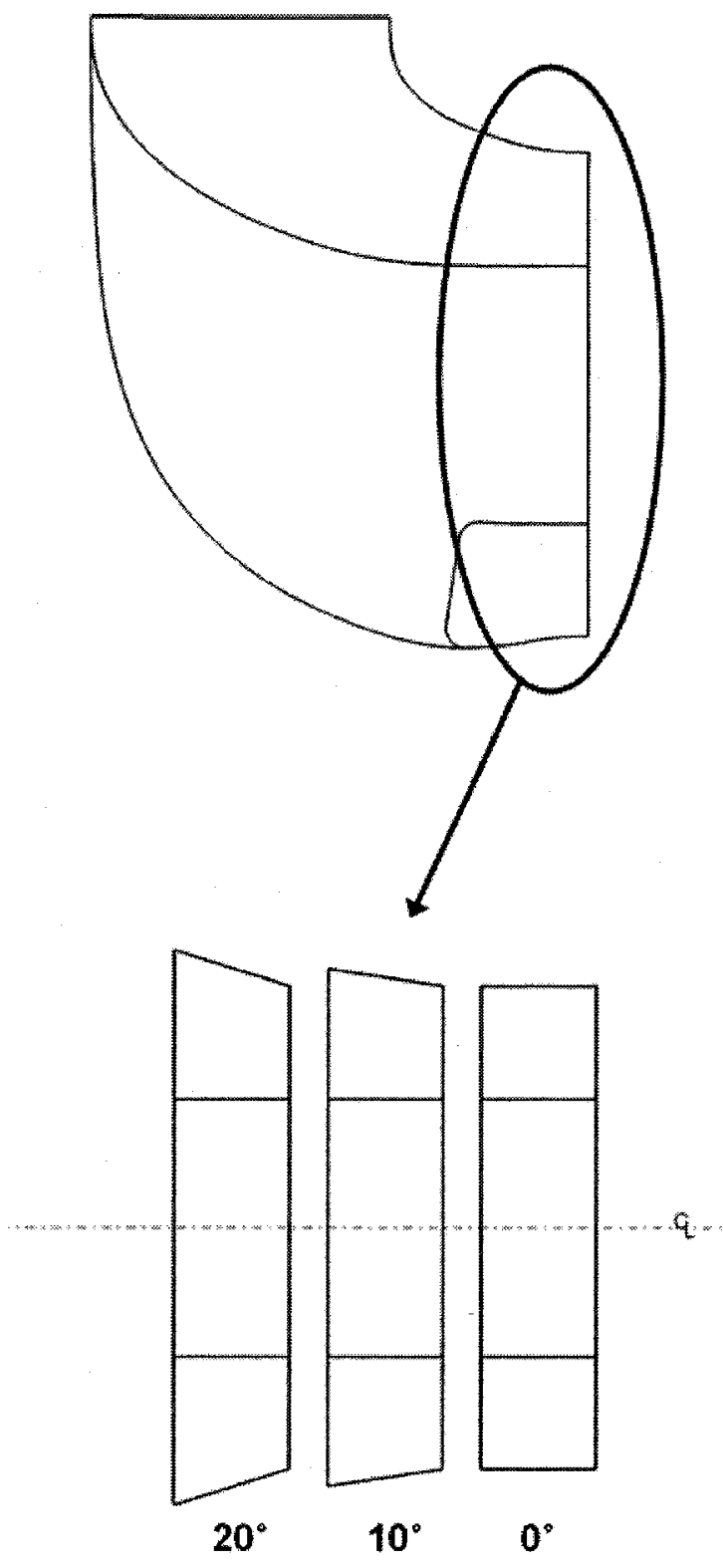

Figure 3-1: Equivalent Cone Diffusion Angle Levels Studied 

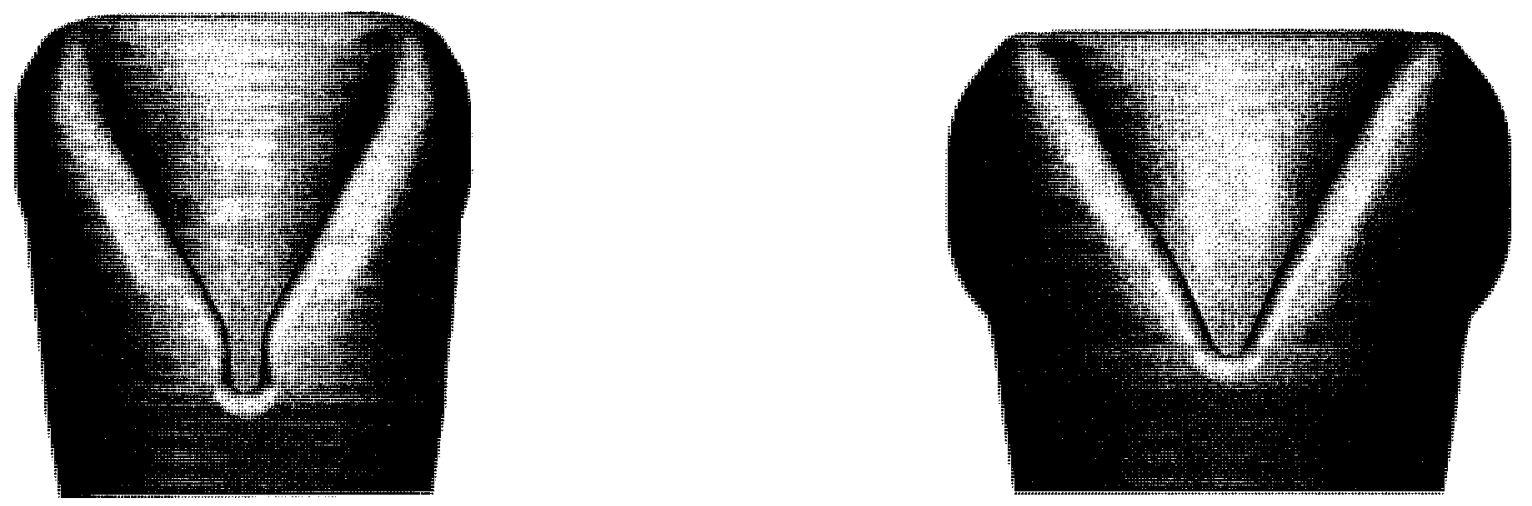

$10^{\circ}$ Wedge Angle
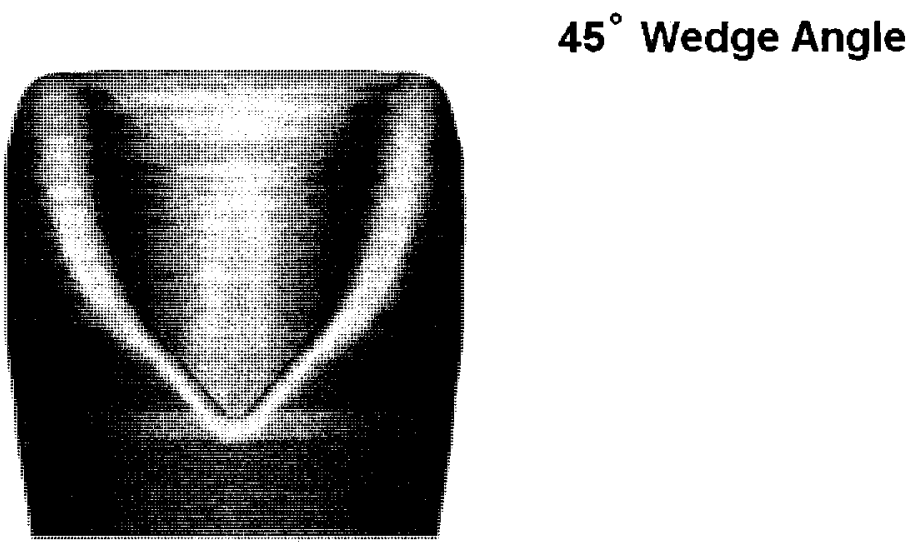

\section{$80^{\circ}$ Wedge Angle}

Figure 3-2: Duct Wedge Angle Levels Studied 

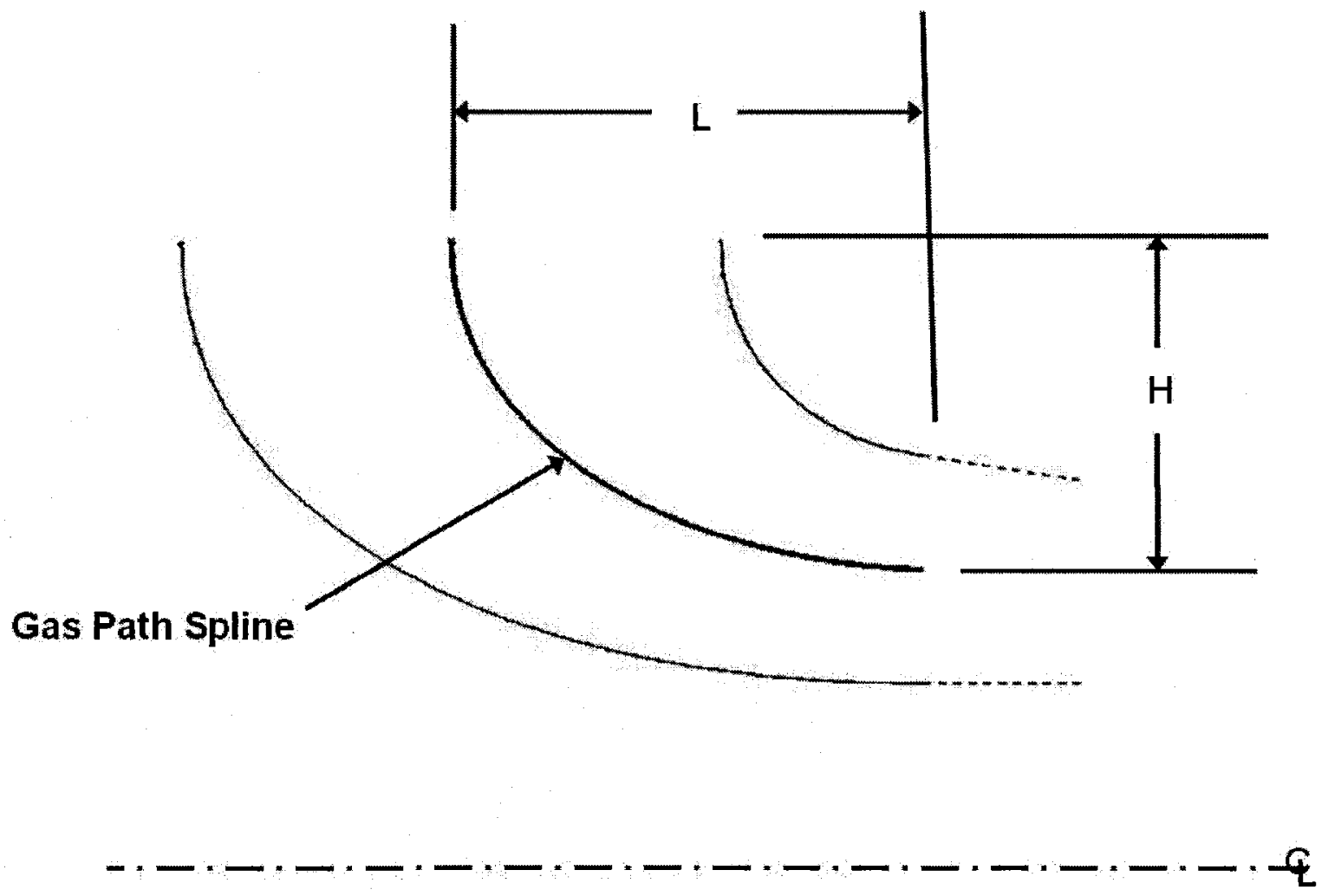

Figure 3-3: Gas Path Aspect Ratio

\subsubsection{Gas Path Aspect Ratio}

The annular to rectangular region of the exhaust duct includes a $90^{\circ}$ bend where the flow will have a strong tendency to separate from the inner curve. While it is not possible to avoid making the $90^{\circ}$ bend, it is possible to adjust the length of duct over which the transition will occur. It is expected that a short duct will lead to severe flow separation along the inner curve as a result of an increased pressure gradient. Longer ducts will have lower pressure gradients reducing or delaying flow separation. The length of the annular to rectangular transition region can be quantified through Eq. 3.2 which adds the duct height as an additional parameter. This aspect ratio is the ratio of axial length, $L$, over radial height, $H$, of a spline curve representing the general flow direction. The length and height are measured between the start and end points of the gas path spline as demonstrated in Fig. 3-3. 

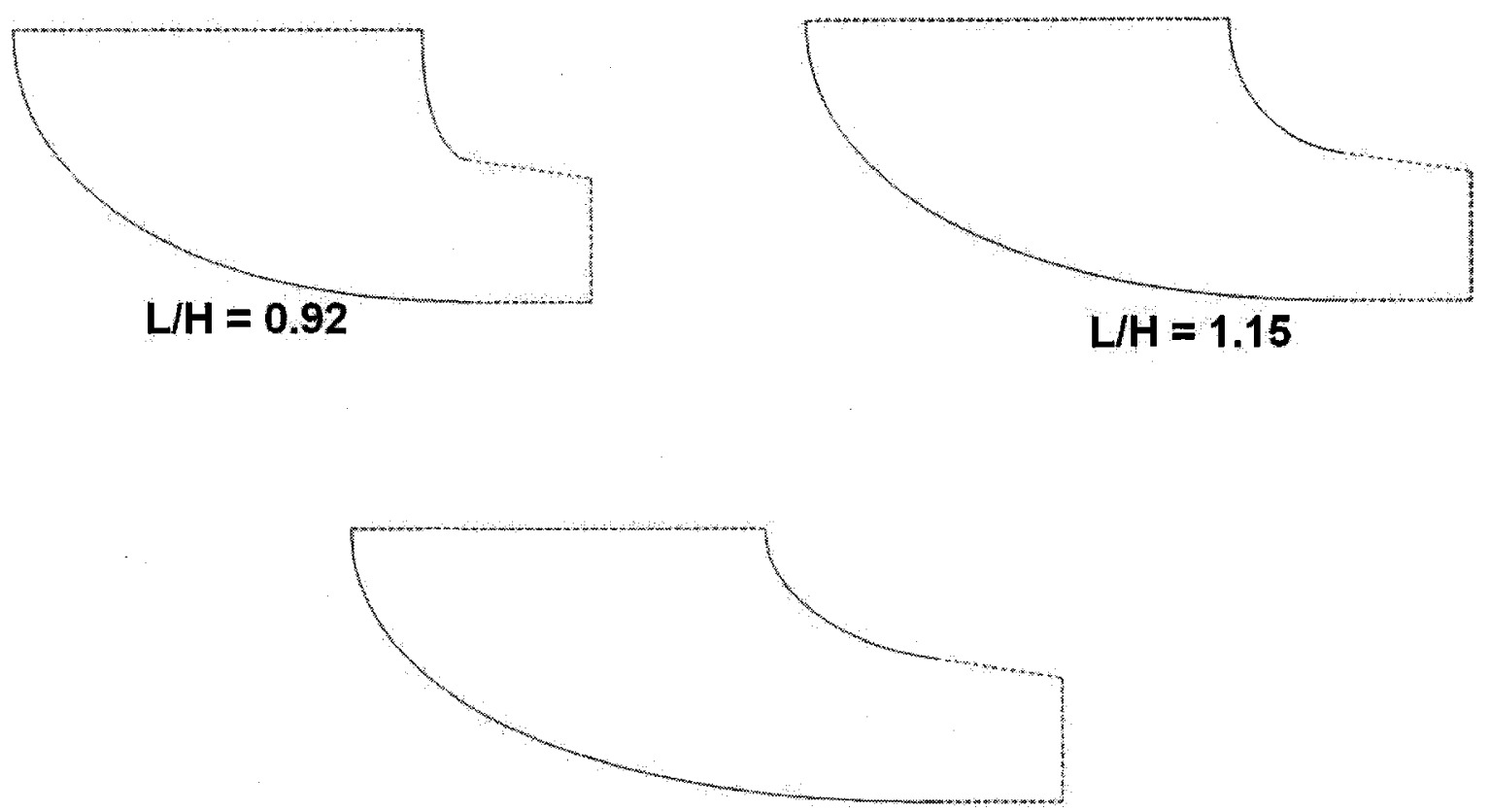

\section{$L / H=1.38$}

Figure 3-4: Gas Path Aspect Ratio Levels Studied

$$
A S=\frac{L}{H}
$$

For this study, the height has been fixed while the length varied. The mean gas path aspect ratio used is 1.15 which is representative of the sample P\&WC duct. To ensure that flow separation occurs, an aspect ratio of 0.92 was selected as the minimum because of the aggressive turn along the inner curve. An aspect ratio of 1.38 was selected as the maximum with the expectation that flow will remain attached along the inner curve. Figure 3-4 demonstrates these aspect ratios where each cross-section was created with an ECDA of $10^{\circ}$ and the same exit area.

\subsubsection{Annular to Rectangular Transition Region}

An additional factor affecting the pressure gradient along the annular to rectangular transition is the remaining area ratio between the flow splitter and the duct exit, see Fig. 3-5 


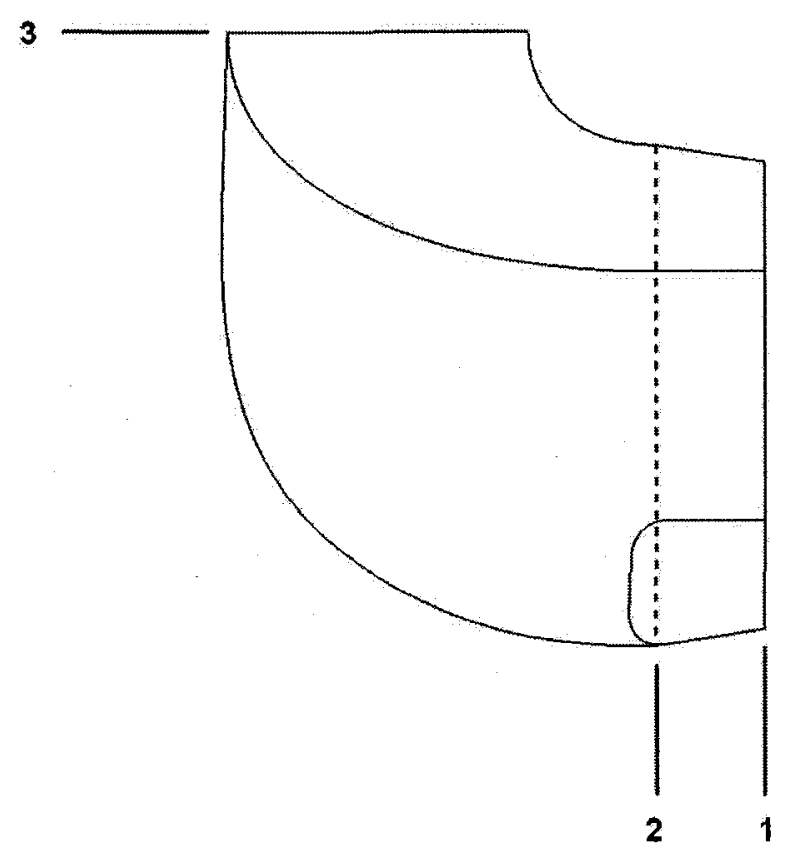

Figure 3-5: Duct Locations where Area is Specified

section 2 to 3 . For a constant duct aspect ratio, any change in the area ratio will affect the streamwise pressure gradient. Small area ratio ducts will have less diffusion and therefore the smallest streamwise pressure gradient. Large area ratio ducts will have increased diffusion, a large streamwise pressure gradient, and increase the possibility of flow separation due to the increased boundary layer growth. The area ratios of the annular to rectangular transition region have been selected to give, once combined with the annular region, Fig. 3-5 section 1 to 2, overall area ratios that do not exceed more then 2 from duct inlet to duct exit, Fig. $3-5$ section 1 to 3 . Table 3.1 lists the combined area ratios of the annular section with the annular to rectangular section to give overall duct area ratios.

\subsubsection{Exhaust Stubs}

Swept exhaust ducts can be found in turboprop and turboshaft configurations that require the engine to be mounted to the aircraft with the output shaft pointed in the fore or aft direction. The end result is to have the exhaust gases leave the engine through an exhaust 
Table 3.1: Exhaust Duct Area Ratio (referenced to figure 3-5)

\begin{tabular}{rrrr} 
ECDA & $A R_{1-2}$ & $A R_{2-3}$ & $A R_{1-3}$ \\
\hline $0^{\circ}$ & 1.000 & 1.000 & 1.000 \\
$10^{\circ}$ & 1.000 & 1.175 & 1.175 \\
$20^{\circ}$ & 1.000 & 1.350 & 1.350 \\
$0^{\circ}$ & 1.180 & 1.000 & 1.180 \\
$10^{\circ}$ & 1.180 & 1.175 & 1.387 \\
$20^{\circ}$ & 1.180 & 1.350 & 1.593 \\
$0^{\circ}$ & 1.450 & 1.000 & 1.450 \\
$10^{\circ}$ & 1.450 & 1.175 & 1.704 \\
$20^{\circ}$ & 1.450 & 1.350 & 1.958
\end{tabular}

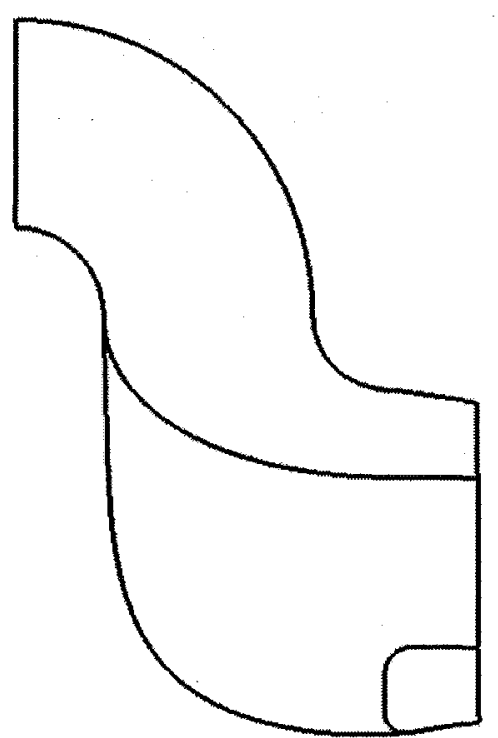

Pusher

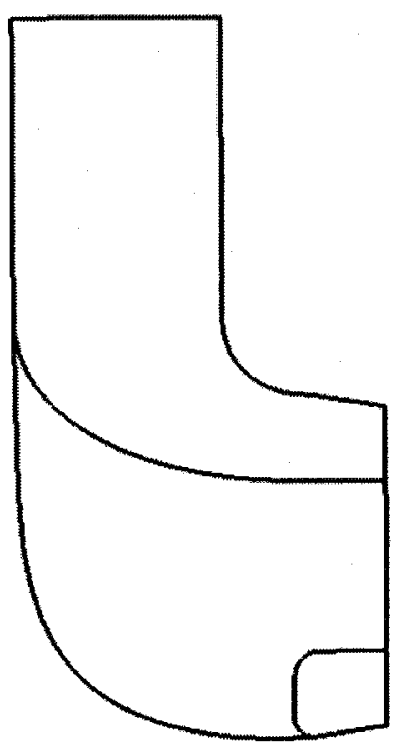

Intermediate

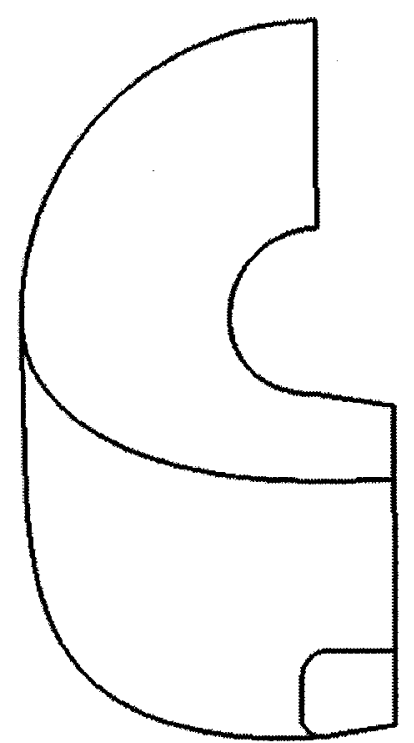

Tractor

Figure 3-6: Exhaust Stub Direction 
stub in a direction which will be dictated by the airframe manufacturer. Three exhaust stub configurations are modelled in this study to account for the possible change in the duct losses with stub direction. Two of the configurations turn the flow to create a Pusher (S-Shape Duct) and Tractor (C-Shape Duct) application while the third takes the exhaust gases straight out (Intermediate-Shaped Duct) defining a transitional point, Fig. 3-6. For each exhaust stub configuration, the cross-sectional area and length was maintained constant, while for the turning exhaust stubs, the turning radius, was maintained constant.

\subsection{Aerodynamic Design Space}

\subsubsection{Swirl}

Moderate inlet swirl has been shown to be beneficial in achieving optimum diffusion in two dimensional diffusers. Annular diffusers can be designed with a large ECDA (ie. short ducts with large diffusion angles), resisting flow separation when swirl is present at the diffuser inlet. For the current study, the effect of swirl on diffuser performance is not as apparent as it is in two dimensional diffusers. While inlet swirl should still be beneficial in the annular portion of the exhaust duct, the flow splitter performance, however, will not benefit from large incidence angles and the annular to rectangular region of the exhaust duct will result in an asymmetric flow field.

Some inlet swirl angle profiles of several similar exhaust ducts can be seen in Fig. 3-7. These profiles are produced at P\&WC using an in-house code which neglects the upstream effect of the downstream diffuser and predicts a circumferentially uniform flow distribution. While many profiles can be observed in Fig. 3-7, it was decided that the swirl gradient used in the current study would be of a constant gradient which fits within the given sample, identified in Fig. 3-7 as the dotted line. This swirl gradient varies by $8^{\circ}$ from $r_{i}$ to $r_{o}$ and is identified by the swirl value found crossing mid way along the annulus height, Fig. 3-7 shows nominal $0^{\circ}$. The range of nominal swirl angles selected to be studied varies from nominal $0^{\circ}$ to $35^{\circ}$. 


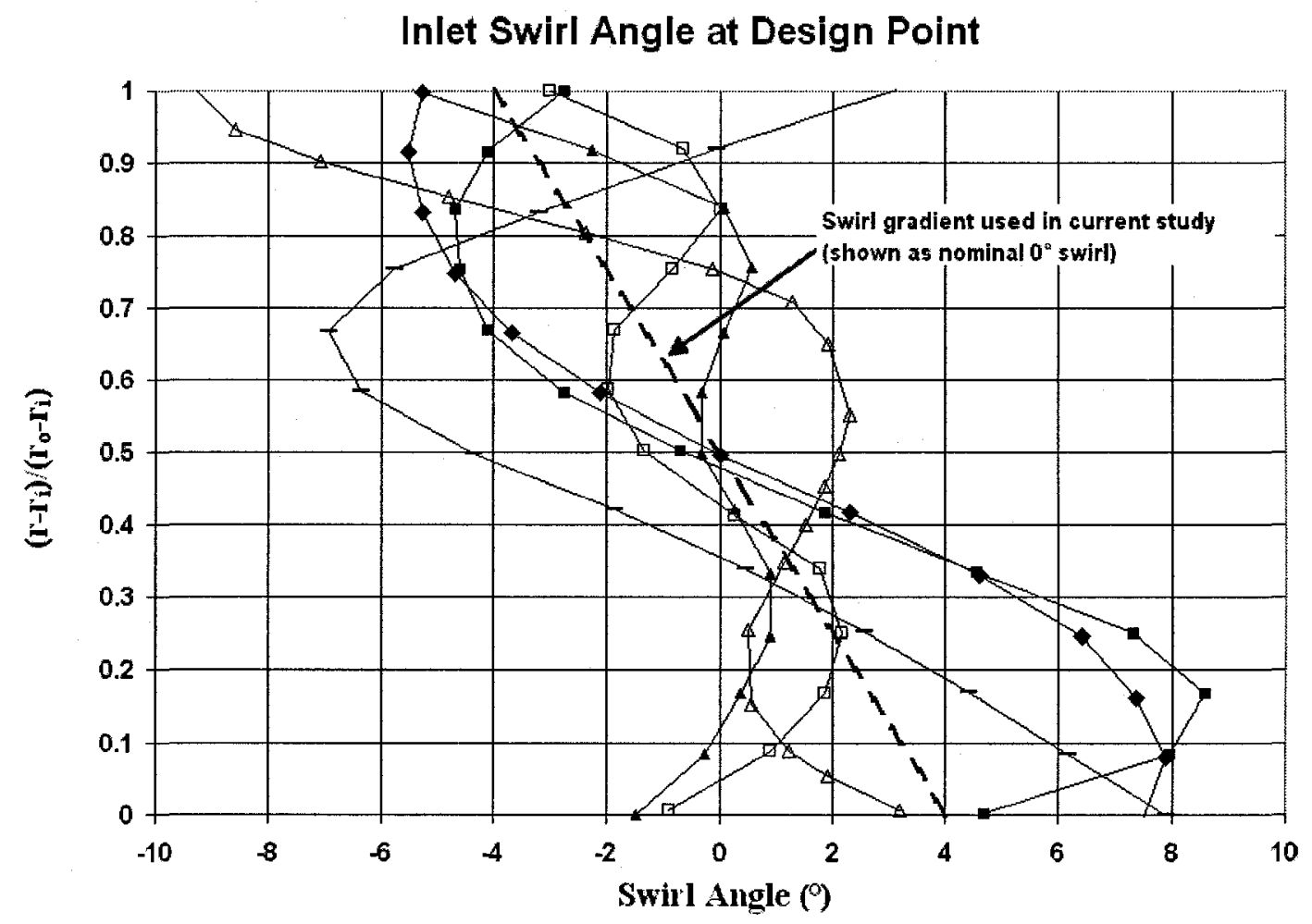

Figure 3-7: Sample of exhaust duct inlet swirl gradients 


\subsubsection{Inlet Boundary Layer Blockage}

It is fully evident from Chapter 2 that inlet boundary layer blockage defined by

$$
\text { Blockage }=\frac{\int_{A} \frac{u}{U} d A}{\int_{A} d A} \times 100
$$

has a significant effect on diffuser performance. With regards to the diffusers in this study, it is expected that the duct pressure losses will increase with increasing inlet boundary layer blockage. The presence of an adverse pressure gradient will result in further increasing the boundary layer thickness as flow travels downstream from the exhaust duct inlet. As the boundary layer thickness grows, the wall shear stress reduces due to a decreasing velocity gradient in the direction normal to the wall. Flow separation will occur as the wall shear stress approaches zero, contributing further to the exhaust duct losses. A turbulent inlet boundary layer will either prevent or delay the onset of flow separation. In the current work, three values of boundary layer blockage have been studied and are displayed in Fig. 3-8. The largest inlet blockage expected to exist in practice is $10 \%$. The presence of the turbine upstream of the exhaust duct inlet will likely produce a turbulent boundary layer, therefore the minimum expected inlet blockage will be less then $1 \%$. An inlet boundary layer blockage between $4 \%$ and $8 \%$ will give a good mean value for correlating inlet boundary layer blockage later on in this study. The $1 / 7^{\text {th }}$ power law given by

$$
\frac{u}{U}=\left(\frac{r}{\delta}\right)^{\frac{1}{7}}
$$

has been used to define the velocity gradient within the boundary layer.

\subsection{Full Factorial Design}

To find the sensitivity of each of the geometric and aerodynamic parameters on exhaust duct performance requires the modelling of numerous exhaust duct cases covering every possible combination of parameters. This approach to performing a sensitivity analysis is termed a Full Factorial Design requiring tremendous resources to complete a timely analysis. To put this into perspective, this study has identified five geometric parameters having a first order 
Inlet Boundary Layer

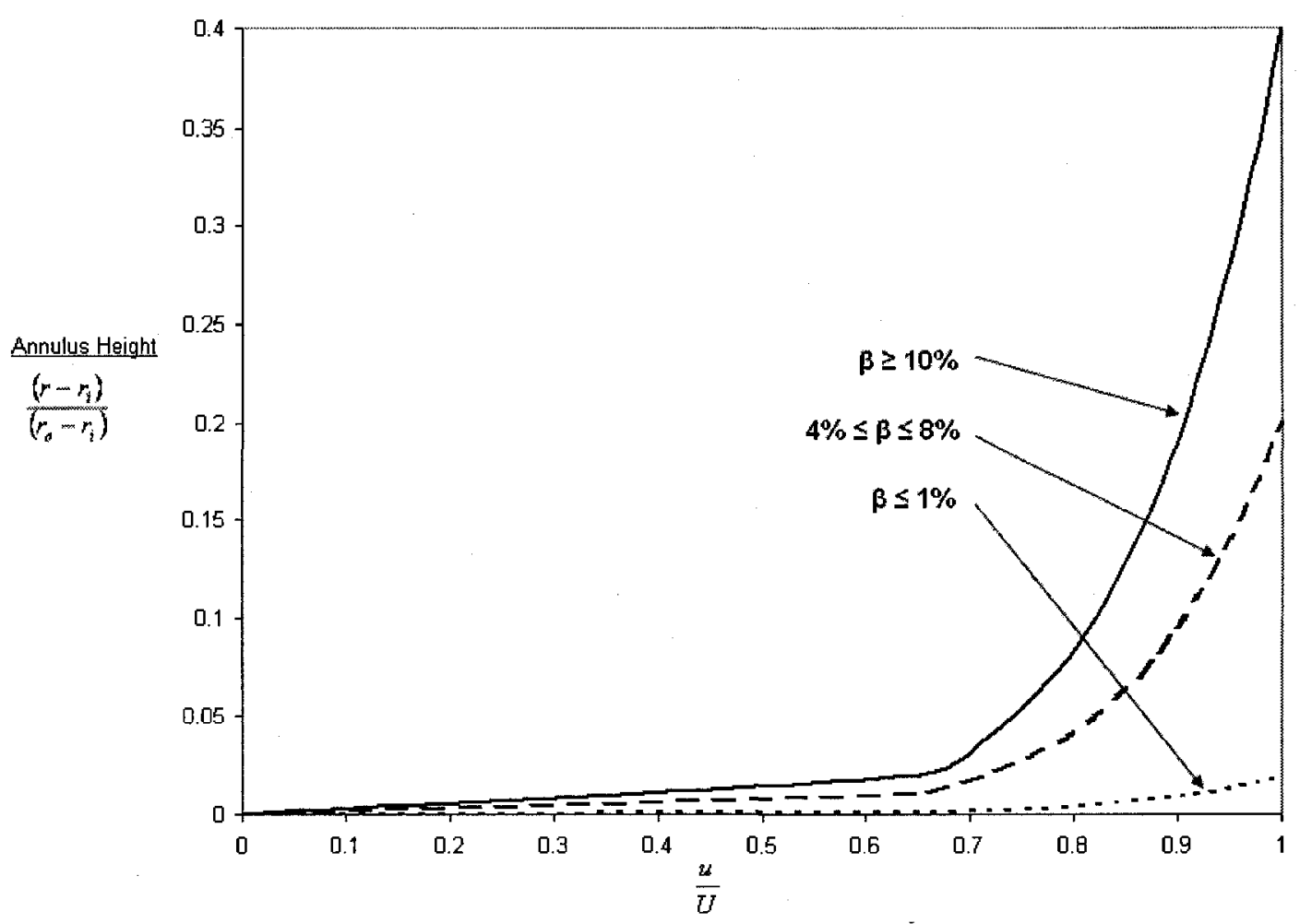

Figure 3-8: Boundary layer axial velocity profiles 
Table 3.2: Geometric Parameters

\begin{tabular}{lllc} 
& Geometric Parameter & Levels & Number of Levels \\
\hline 1) & ECDA & $0^{\circ}, 10^{\circ}, 20^{\circ}$ & 3 \\
2) & Flow Splitter Wedge Angle & $10^{\circ}, 45^{\circ}, 80^{\circ}$ & 3 \\
$3)$ & AS & $0.92,1.15,1.38$ & 3 \\
$4)$ & $A R_{2-3}$ & $1.000,1.175,1.350$ & 3 \\
5) & Stub Direction & S-Shape, C-Shape, Straight & 3 \\
\cline { 2 - 3 } & & Total Combinations = & 243
\end{tabular}

Table 3.3: Aerodynamic Parameters

\begin{tabular}{lllc} 
& Aerodynamic Parameter & Levels & Number of Levels \\
\hline 1) & Inlet Swirl Angle & $0^{\circ}, 10^{\circ}, 25^{\circ}, 35^{\circ}$ & 4 \\
2) & Inlet Boundary Layer Blockage & Low, Med, High & 3 \\
\cline { 3 - 3 } & & Total Combinations $=$ & 12
\end{tabular}

effect on exhaust duct performance, where a minimum of three values are needed for each parameter in order to find a non-linear effect on exhaust duct performance. Additionally, each exhaust duct would need to be modelled using each combination of inlet swirl angle and inlet boundary layer blockage. A full-factorial design with five parameters each having three values will require $3^{5}=243$ exhaust ducts to analyze as seen in Table 3.2. Each of these exhaust ducts would then be modelled using each of the aerodynamic parameters in Table 3.3 giving 12 aerodynamic boundary conditions. The total combination of geometric and aerodynamic parameters which need to be modelled is 2916 , which is an unpractical task to perform. Consequently methods of selecting the minimum number of experiments required to give the full information about each factor exist and are called partial-fraction designs [21]. 


\subsection{Taguchi Design}

Dr. Genichi Taguchi [21] has created a set of guidelines for performing partial-fraction designs using a special set of arrays called orthogonal arrays. In an orthogonal array all levels of all factors are represented an equal number of times, and the combinations of any two factors are also represented an equal number of times. In this respect, an orthogonal array can be viewed as being well balanced, providing equal pairing between independent parameters therefore reducing the total combination of parameters needed to determine their effect on the dependent variable.

\subsubsection{Assumption}

Prior to choosing the orthogonal array, we must understand the assumption that is being made in the Taguchi Design. The assumption is that the factors that are selected are independent of each other and can be separated. Any interactions are assumed to have a higher order effect on the dependent parameter and are confounded within the main effects. The objective of this study is to catch the first order effects on exhaust duct losses. While some interactions are expected to exist, they are assumed to have a smaller influence on the exhaust duct losses then do the independent effects.

\subsubsection{Interactions}

Interactions that can be expected are upstream parameters affecting the downstream parameters, such as flow separation in the annulus on the flow splitter and the annular to rectangular transition region. It is not expected that a separated flow will interact with the downstream parameters at the end-walls the same way as an attached flow does because of changes in the boundary layer, however the stream-wise momentum is going to be an order of magnitude larger and will continue to relate stronger to the first order duct losses. There is one particular interaction that should not be neglected in this study, namely the interaction between the exhaust duct and the exhaust stub direction because the same exhaust duct can be used in both pusher and tractor configurations, Fig. 3-6. 


\subsubsection{Selecting an Orthogonal Array}

An orthogonal array is selected by first considering the independent parameters one through four in table 3.4. Each of the four parameters consist of three levels, therefore the minimum number of geometries needed can be defined to be:

$$
N_{\text {Taguchi }}=1+\sum_{i=1}^{N V}\left(L_{i}-1\right)
$$

Where:

$N V=$ The number of parameters

and

$L_{i}=$ The number of levels for parameter $i$

For this study $N V$ is equal to four and $L_{i}$ is equal to three for each of the parameters giving nine geometries. An $L_{9}$ orthogonal array shown in Table 3.4 is well suited for this study. To include the interaction with exhaust stub direction, the $L_{9}$ orthogonal array is expanded to allow each of the nine geometries to be combined with all stub directions yielding 27 geometries to study. Table 3.5 presents the expanded $L_{9}$ orthogonal array with the physical variable to give exhaust duct families A through G. It can be well observed that the Taguchi design has reduced the amount of geometries required for the sensitivity analysis from 243 in the full factorial design to 27 in the partial factorial design. 
Table 3.4: $L_{9}$ Orthogonal Array

Independent Parameter

\begin{tabular}{ccccc} 
Build & Parameter 1 & Parameter 2 & Parameter 3 & Parameter 4 \\
\hline 1 & 1 & 1 & 1 & 1 \\
2 & 1 & 2 & 2 & 2 \\
3 & 1 & 3 & 3 & 3 \\
4 & 2 & 1 & 2 & 3 \\
5 & 2 & 2 & 3 & 1 \\
6 & 2 & 3 & 1 & 2 \\
6 & 3 & 1 & 3 & 2 \\
8 & 3 & 2 & 1 & 3 \\
9 & 3 & 3 & 2 & 1
\end{tabular}


Table 3.5: $L_{9}$ Orthogonal Array Expanded for Stub Direction

Flow Splitter

Duct ECDA Wedge Angle $A R_{2-3} \quad$ AS Stub Direction

\begin{tabular}{cccccc}
\hline \hline A-1 & $0^{\circ}$ & $10^{\circ}$ & 1.000 & 0.92 & C-Shape \\
A-2 & $0^{\circ}$ & $10^{\circ}$ & 1.000 & 0.92 & S-Shape \\
A-3 & $0^{\circ}$ & $10^{\circ}$ & 1.000 & 0.92 & Straight \\
\hline B-1 & $0^{\circ}$ & $45^{\circ}$ & 1.175 & 1.15 & C-Shape \\
B-2 & $0^{\circ}$ & $45^{\circ}$ & 1.175 & 1.15 & S-Shape \\
B-3 & $0^{\circ}$ & $45^{\circ}$ & 1.175 & 1.15 & Straight \\
\hline C-1 & $0^{\circ}$ & $80^{\circ}$ & 1.350 & 1.38 & C-Shape \\
C-2 & $0^{\circ}$ & $80^{\circ}$ & 1.350 & 1.38 & S-Shape \\
C-3 & $0^{\circ}$ & $80^{\circ}$ & 1.350 & 1.38 & Straight \\
\hline D-1 & $10^{\circ}$ & $10^{\circ}$ & 1.175 & 1.38 & C-Shape \\
D-2 & $10^{\circ}$ & $10^{\circ}$ & 1.175 & 1.38 & S-Shape \\
D-3 & $10^{\circ}$ & $10^{\circ}$ & 1.175 & 1.38 & Straight \\
\hline E-1 & $10^{\circ}$ & $45^{\circ}$ & 1.350 & 0.92 & C-Shape \\
E-2 & $10^{\circ}$ & $45^{\circ}$ & 1.350 & 0.92 & S-Shape \\
E-3 & $10^{\circ}$ & $45^{\circ}$ & 1.350 & 0.92 & Straight \\
\hline F-1 & $10^{\circ}$ & $80^{\circ}$ & 1.000 & 1.15 & C-Shape \\
F-2 & $10^{\circ}$ & $80^{\circ}$ & 1.000 & 1.15 & S-Shape \\
F-3 & $10^{\circ}$ & $80^{\circ}$ & 1.000 & 1.15 & Straight \\
\hline G-1 & $20^{\circ}$ & $10^{\circ}$ & 1.350 & 1.15 & C-Shape \\
G-2 & $20^{\circ}$ & $10^{\circ}$ & 1.350 & 1.15 & S-Shape \\
G-3 & $20^{\circ}$ & $10^{\circ}$ & 1.350 & 1.15 & Straight \\
\hline H-1 & $20^{\circ}$ & $45^{\circ}$ & 1.000 & 1.38 & C-Shape \\
H-2 & $20^{\circ}$ & $45^{\circ}$ & 1.000 & 1.38 & S-Shape \\
H-3 & $20^{\circ}$ & $45^{\circ}$ & 1.000 & 1.38 & Straight \\
\hline I-1 & $20^{\circ}$ & $80^{\circ}$ & 1.175 & 0.92 & C-Shape \\
I-2 & $20^{\circ}$ & $80^{\circ}$ & 1.175 & 0.92 & S-Shape \\
I-3 & $20^{\circ}$ & $80^{\circ}$ & 1.175 & 0.92 & Straight \\
& & & & &
\end{tabular}




\section{Chapter 4}

\section{Geometry Synthesis}

The approach discussed here for geometry synthesis has been designed to produce exhaust ducts which can be specified using only key geometric parameters thought to be responsible for the total pressure losses in the exhaust duct. The 3D nature of the single port swept exhaust duct makes its complete geometric representation impossible using only one dimensional geometric parameters. Therefore assumptions are made to complete the geometry. The blanks in the steps discussed in this Chapter have been filled in based on the assumption of how a single port swept exhaust duct should be represented geometrically, and could vary with each designer who uses this approach. It is the assumption of this author that the steps not discussed here would only represent a second order effect on exhaust duct losses, and therefore do not play a crucial role in the current study.

A sample of a single port swept exhaust duct taken from P\&WC is shown in Fig. 4-1. This exhaust duct has smooth flowing features which do not contain any sharp edges which can disturb the flow. To describe the geometric features of this duct would require complex splines and curves which do not suit the present study because of the numerous parameters that would be needed. Steps have been taken to simplify the exhaust duct such that it can be described by simple one dimensional parameters identified in Chapter 3 . 

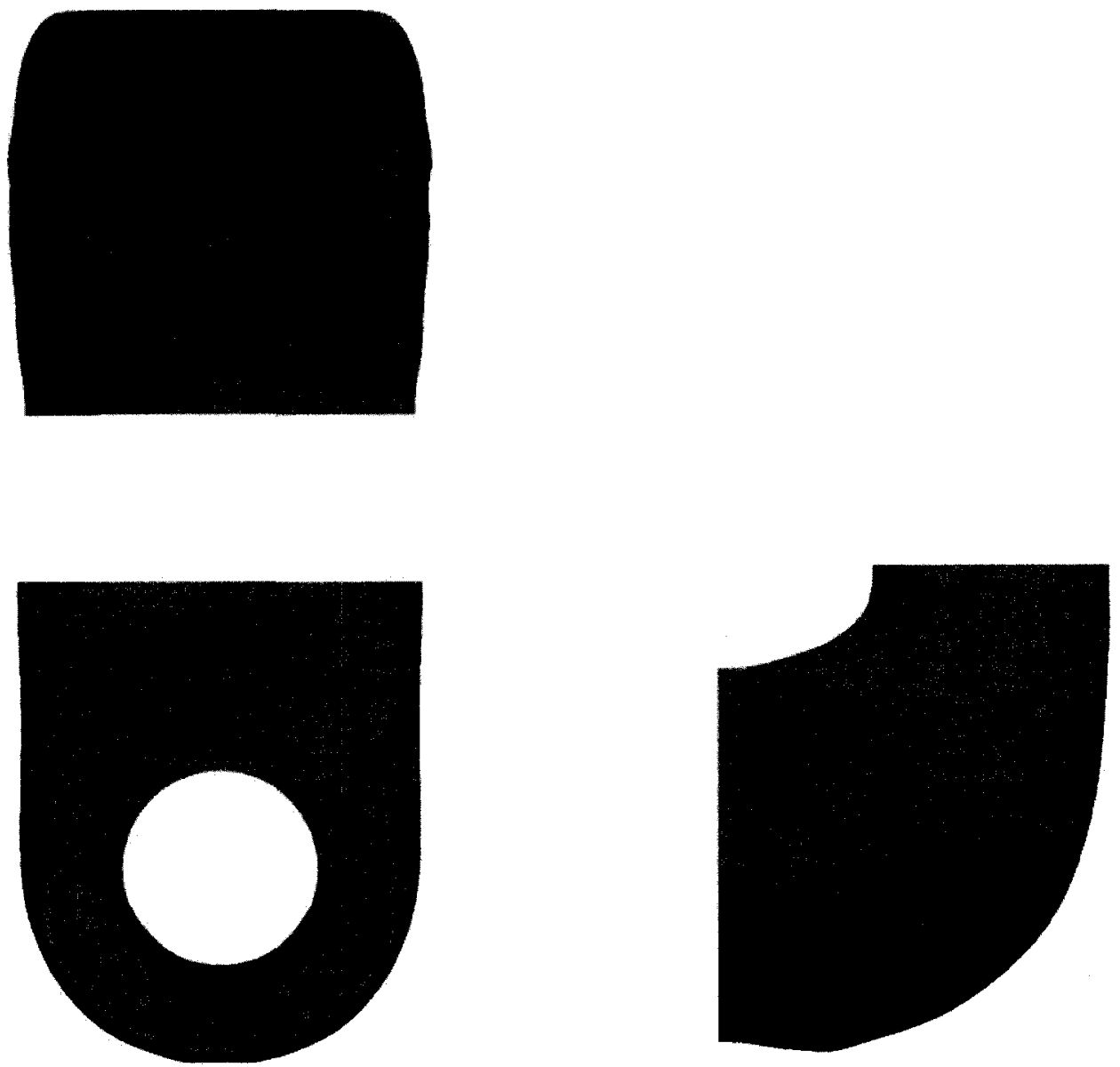

Figure 4-1: Sample P\&WC Single Port Swept Exhaust Duct 

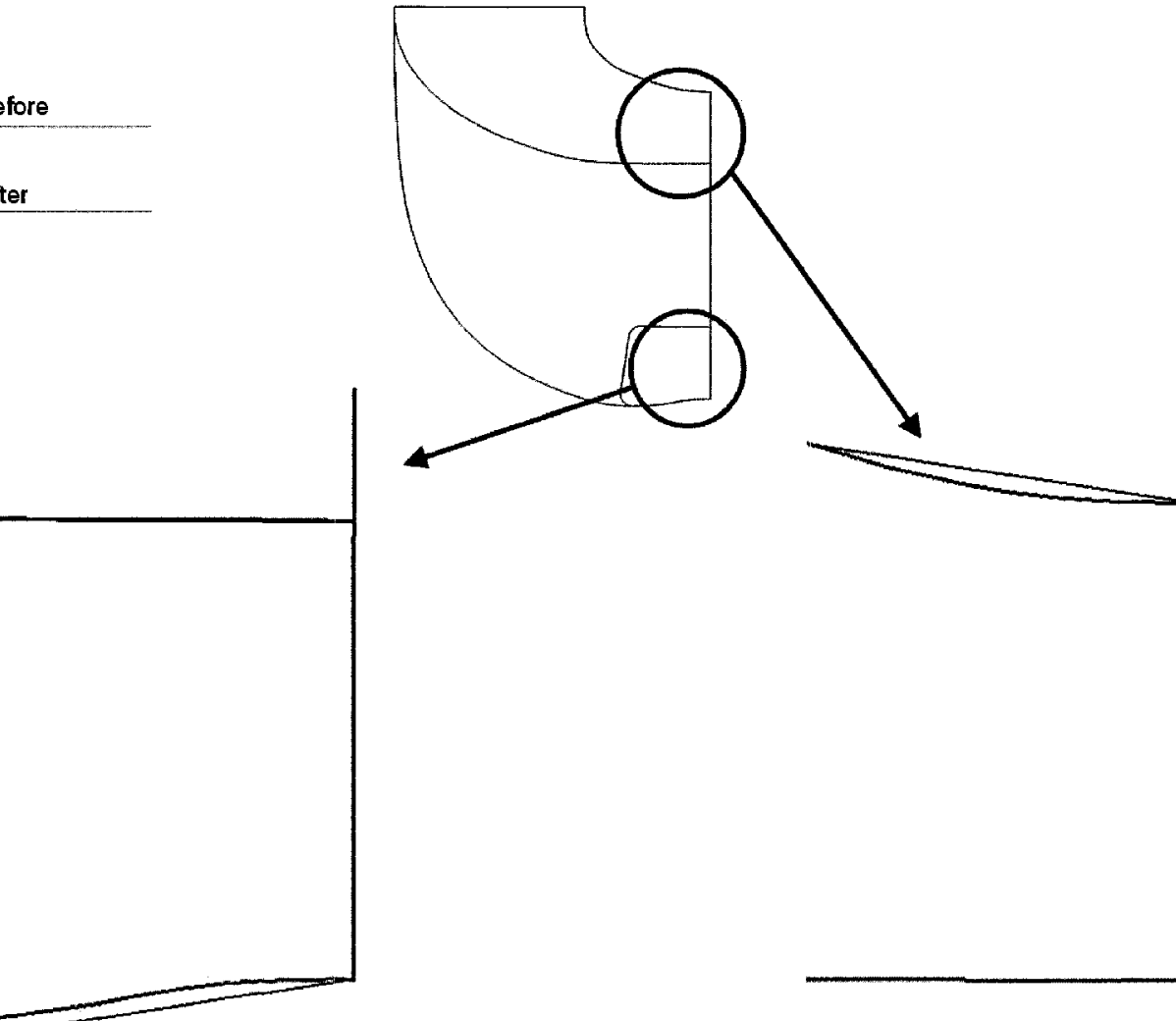

Figure 4-2: Equivalent Cone Diffusion Angle

\subsection{Equivalent Cone Diffusion Angle}

From examination of the annular region of the sample exhaust duct, the inner and outer walls are not straight walled and are found to be represented by splines. The curved profile has been made linear, and is now better suited to be described by Eq. 3.1. Further examination of the sample exhaust duct shows that the hub surface is nearly straight walled with a constant radius and has therefore been replicated this way in the current study. The cross-sectional profile of the annular region is given in Fig. 4-2 which presents the sample geometry with the simplified representation. 


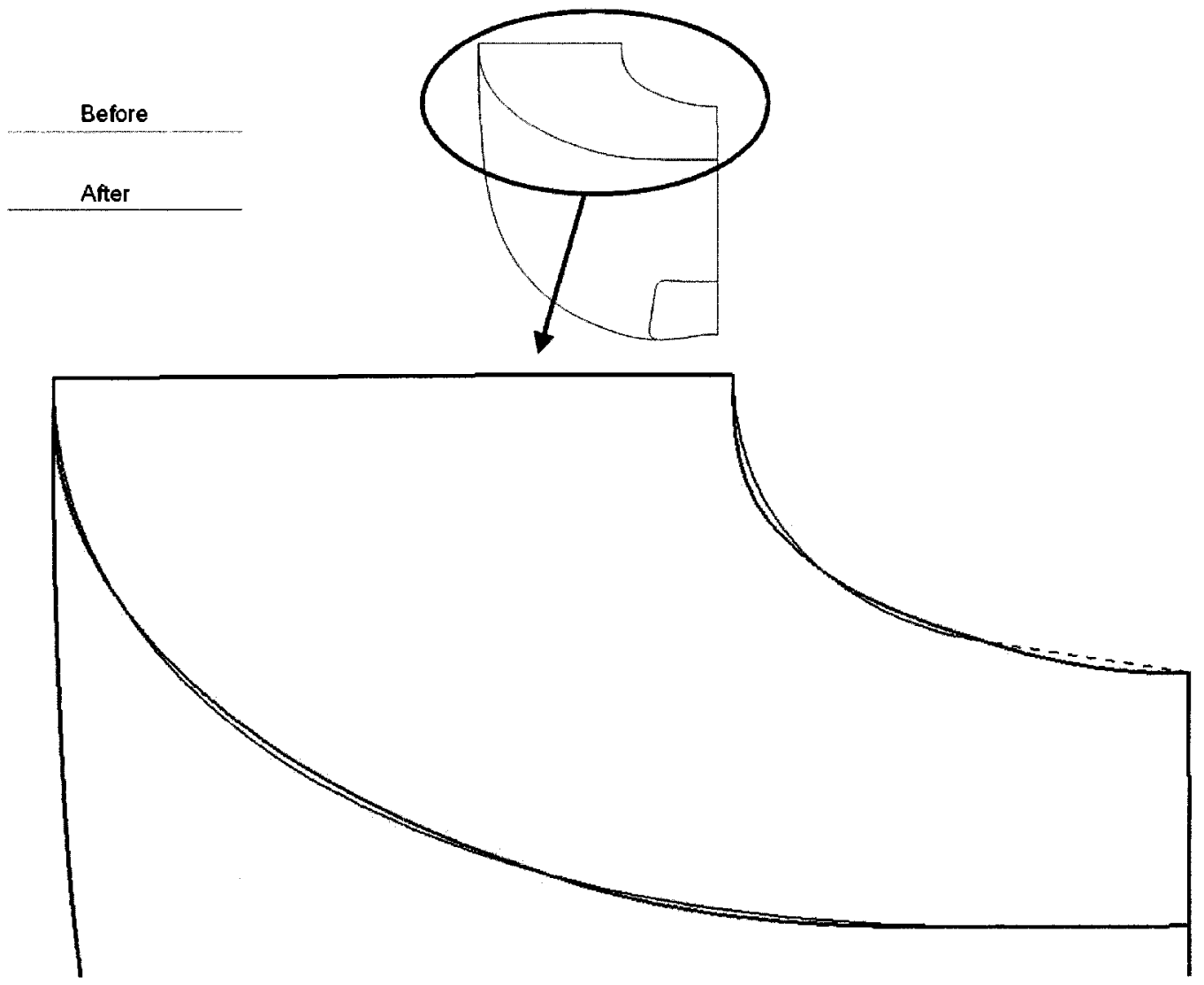

Figure 4-3: Gas Path Conic 


\subsection{Gas Path}

The remaining portion of the gas path continues from the exit of the annular section to the duct exit. The profile of the sample duct can be closely reproduced with the use of conics as shown in Fig. 4-3. The conics used require five parameters; a start point, an end point, a tangent direction at the start point, a tangent direction at the end point, and a conic parameter. The start points and tangent directions are set from the preceding section defined by the ECDA. The end points are defined by the exhaust duct exit area and position. The tangent direction for the end points is always $90^{\circ}$ from the duct inlet. A conic parameter of 0.45 has been chosen based on a good fit with the sample duct and used throughout this study. Once the inner and outer conics have been defined, equally spaced points can be located along each curve and joined with straight lines. A gas path spline can be constructed by locating the midpoints of each line and then connecting them with a spline Fig. 4-4.

\subsection{Gas Path Aspect Ratio}

Once the inner and outer conics have been defined, equally spaced points can be located along each curve and joined with straight lines. A gas path spline can be constructed by locating the midpoints of each line and then connecting them with a spline, Fig. 4-4. The gas path aspect ratio can now be measured according to Eq. 3.2.

\subsection{Flow Splitter Leading Edge}

The flow splitter is located downstream of the annular duct section at the bottom dead center of the exhaust duct, Fig. 4-5). It can be described as being an axisymmetric vane with an elliptical leading edge varying from hub to tip centered along a plane not fully normal to the axial direction. Large fillets are used at hub and tip of the vane to merge smoothly with rest of the domain. The flow splitter smoothly blends from the leading edge outward to the exhaust duct through the annular to rectangular duct transition region.

To construct the flow splitter, a plane is located normal to the gas path spline slightly 


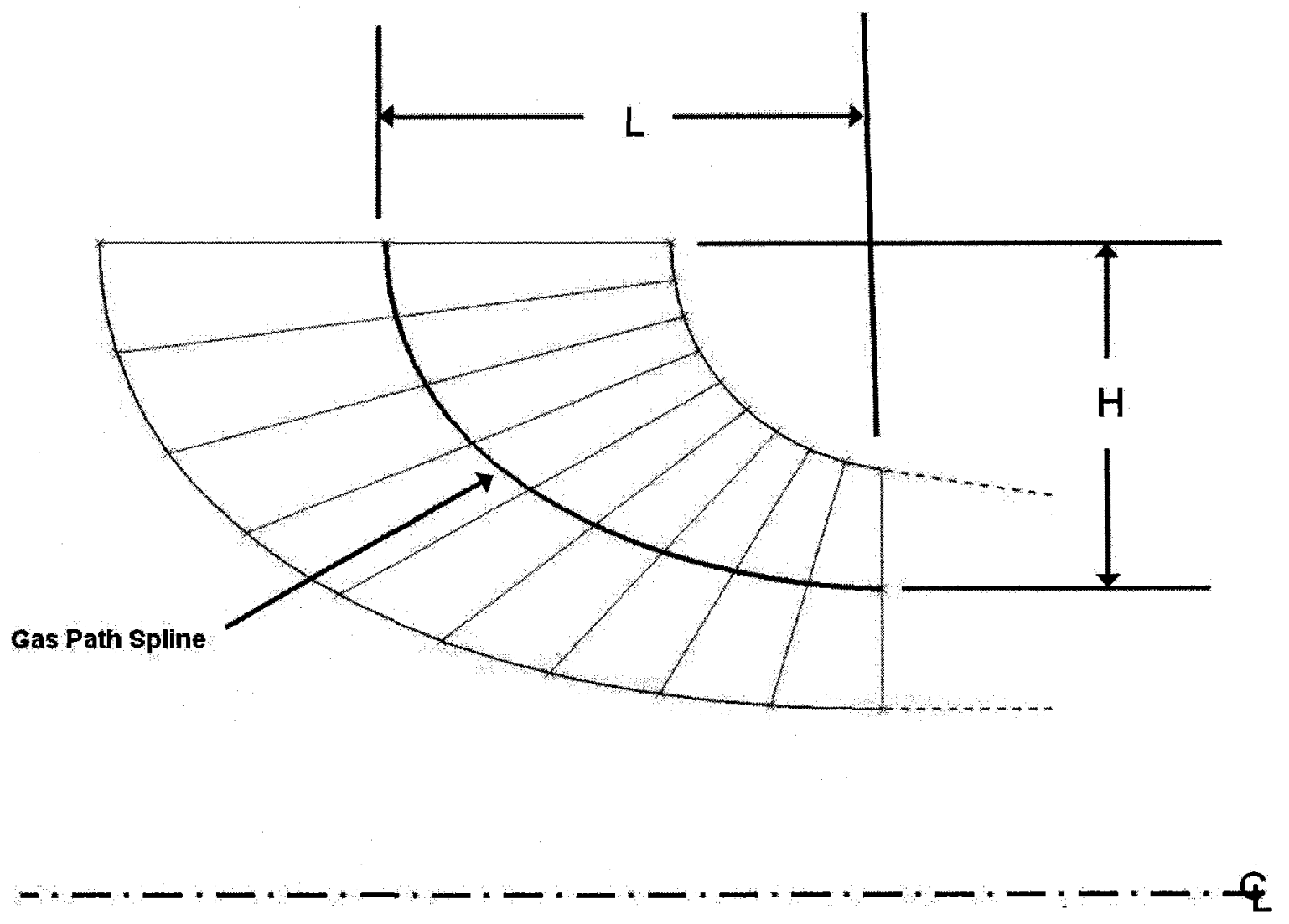

Figure 4-4: Gas Path Spline and Aspect Ratio 


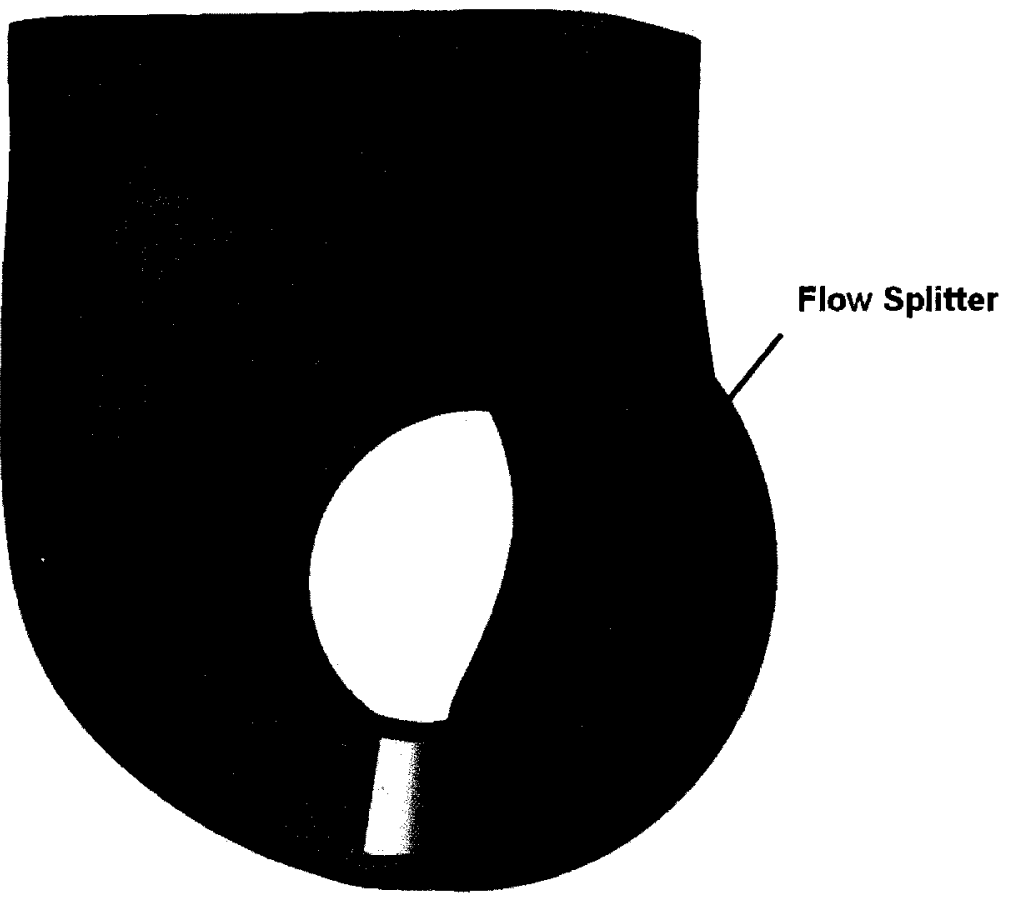

Figure 4-5: Flow Splitter 
downstream of the annular duct segment, Fig. 4-6. With the height of the flow splitter located at the bottom dead center of the duct, it's center is located and a circle is drawn of diameter $D$ in a plain normal to the line defining the flow splitter height. The circle is centered along the symmetry plane of the duct but not constrained to be centered on the line defining the flow splitter height. Two lines are drawn at an angle $\alpha$ intersecting the symmetry line of the flow splitter and tangent to the circle. The circle is allowed to move along the center line with the tangent points falling on the plane positioned normal to the gas path spline. The portion of the circle lying downstream of the plane positioned normal to the gas path is removed leaving the upstream portion to be the leading edge of the flow splitter, Fig. 4-7. The leading edge curve is then extruded to produce the flow splitter which is then joined to the upstream annulus through fillets.

\subsection{Flow Splitter Wedge Angle}

The transition of the exhaust duct geometry from annular to rectangular continues from the leading edge of the flow splitter through a wedge shaped inner passage directing the flow around the hub toward the exhaust duct exit. The P\&WC sample duct shown in Fig. 4-8 demonstrates that the varying leading edge diameter leads to a varying wedge angle from hub angle $\alpha_{1}$ to shroud angle $\alpha_{2}$. The exhaust ducts created in this study contain a single wedge angle as a result of using one leading edge profile from hub to shroud. To smoothly merge the wedge angle into the duct transition section a limit was put on the flow splitter length to allow a smooth transition to occur as seen in the P\&WC sample exhaust duct in Fig. 4-8. This limit was taken as being $1 / 8^{\text {th }}$ the length, $L$, defined in the gas path aspect ratio.

\subsection{Duct Exit Cross-Section}

The sample P\&WC exhaust duct consists of an exit cross-section that is only symmetric across one plane as shown in Fig. 4-9. The duct exit cross-section of the sample P\&WC exhaust has been developed through optimization for a given set of flow conditions which are unknown to this author. For this reason, a fully symmetric exit cross-section, shown as 


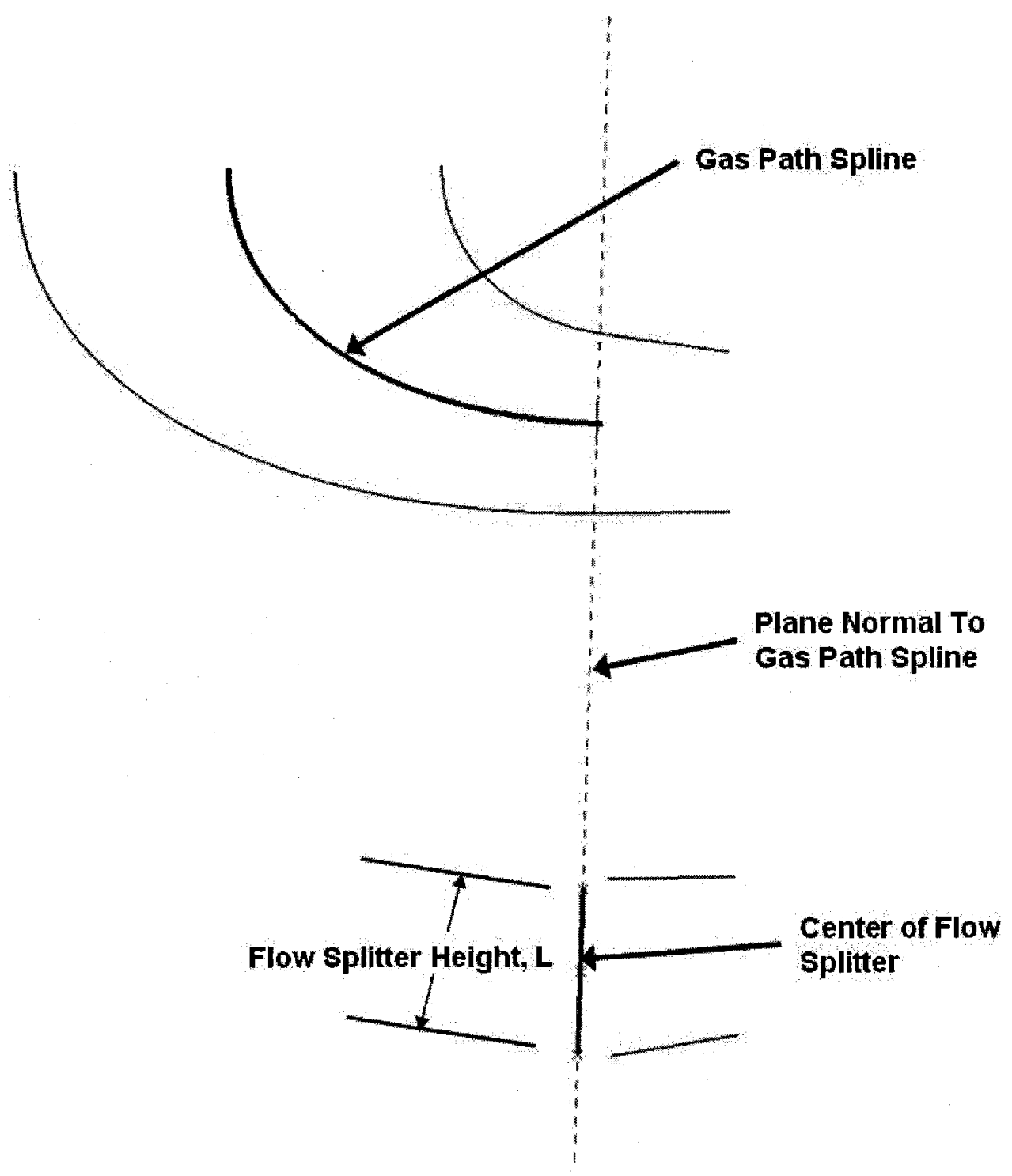

Figure 4-6: Flow Splitter Construction Plane 


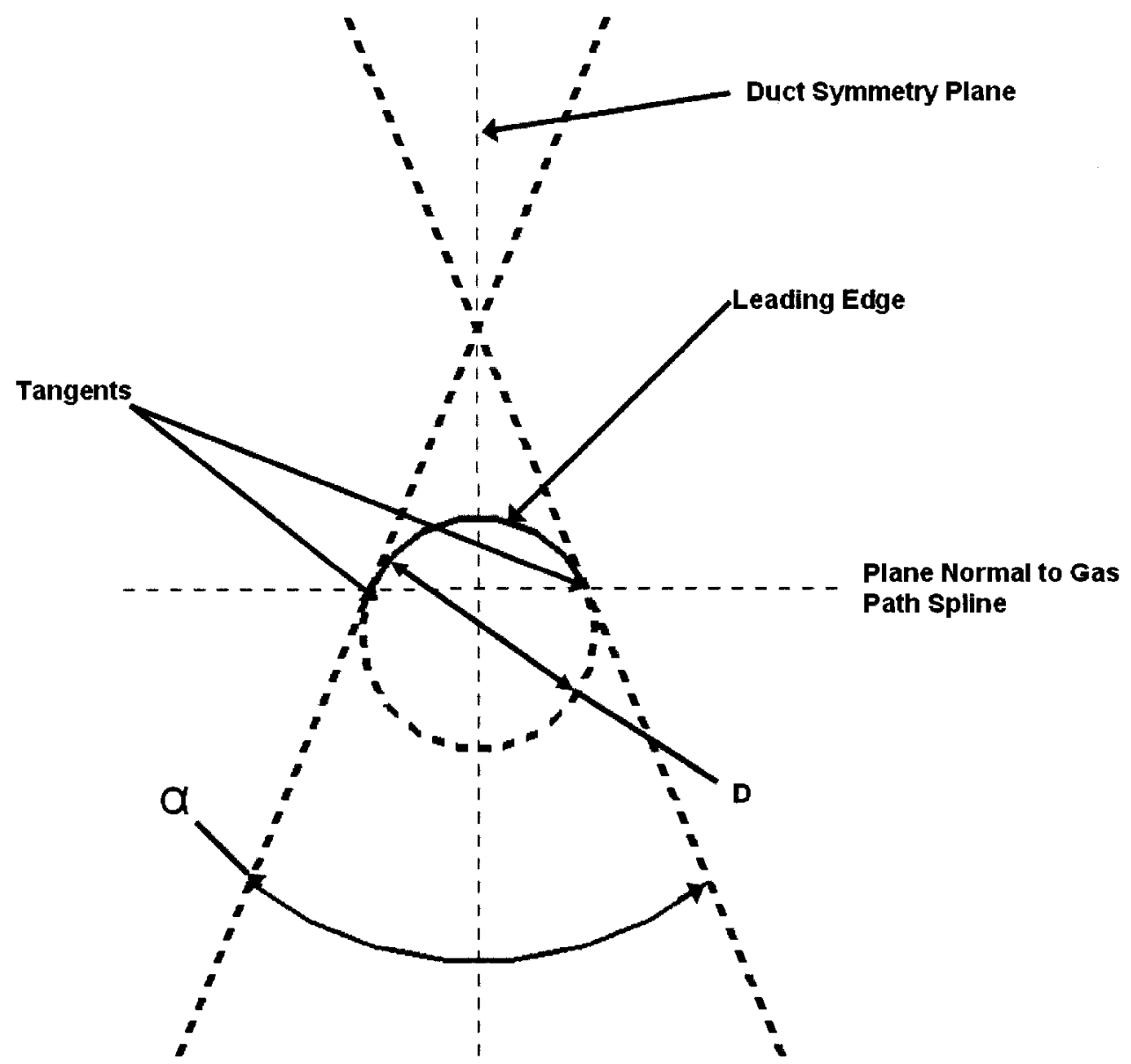

Figure 4-7: Flow Splitter Construction Plane 


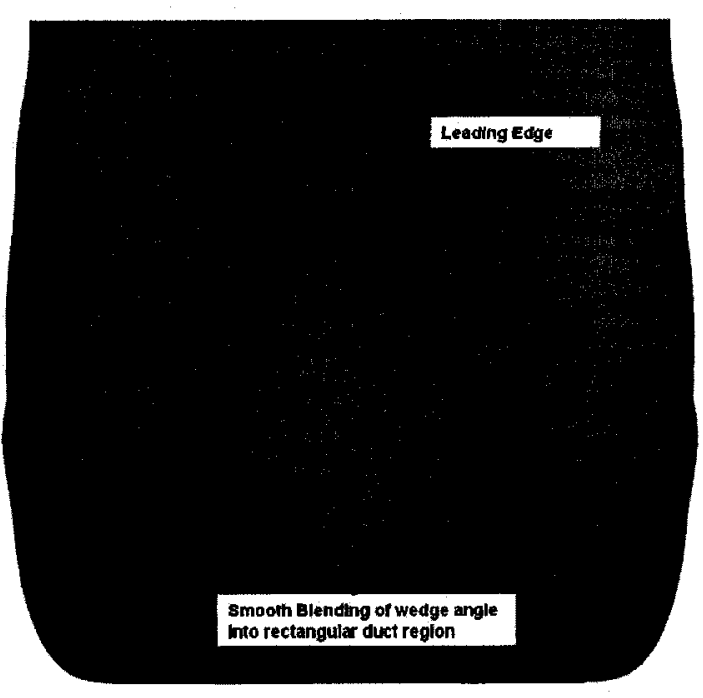

P\&wc Sample Duct

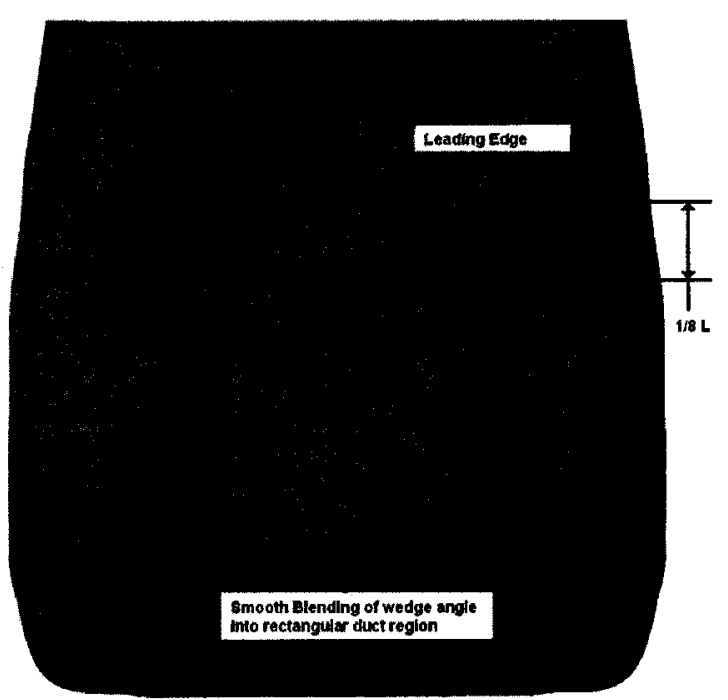

Simplifled Duct Wedge Angle

Figure 4-8: Flow Splitter Wedge Angle

the blue profile in Fig. 4-9, has been used throughout this study so not to introduce any unknown influences produced by the sample P\&WC exhaust duct.

\subsection{Annular to Rectangular Transition Region}

The shape of the exhaust duct from the flow splitter downstream to the exhaust duct exit is defined by cross-section profiles built on planes passing through each of the lines connecting the inner and outer conics shown in Figs. 4-3 and 4-4. The exhaust duct transition from annular to rectangular produces complex cross-sections which cannot be defined with straight lines and curves, resulting in the decision to use splines, Fig. 4-10. Numerous control points are required to define each cross-section spline making it difficult to develop a consistent approach to the design; however, some rules have been created and followed throughout this study. The following rules that have been applied are:

1. The profile is bounded by the annulus hub radius.

2. The profile is bounded so as not to surpass the gas path inner conic. 


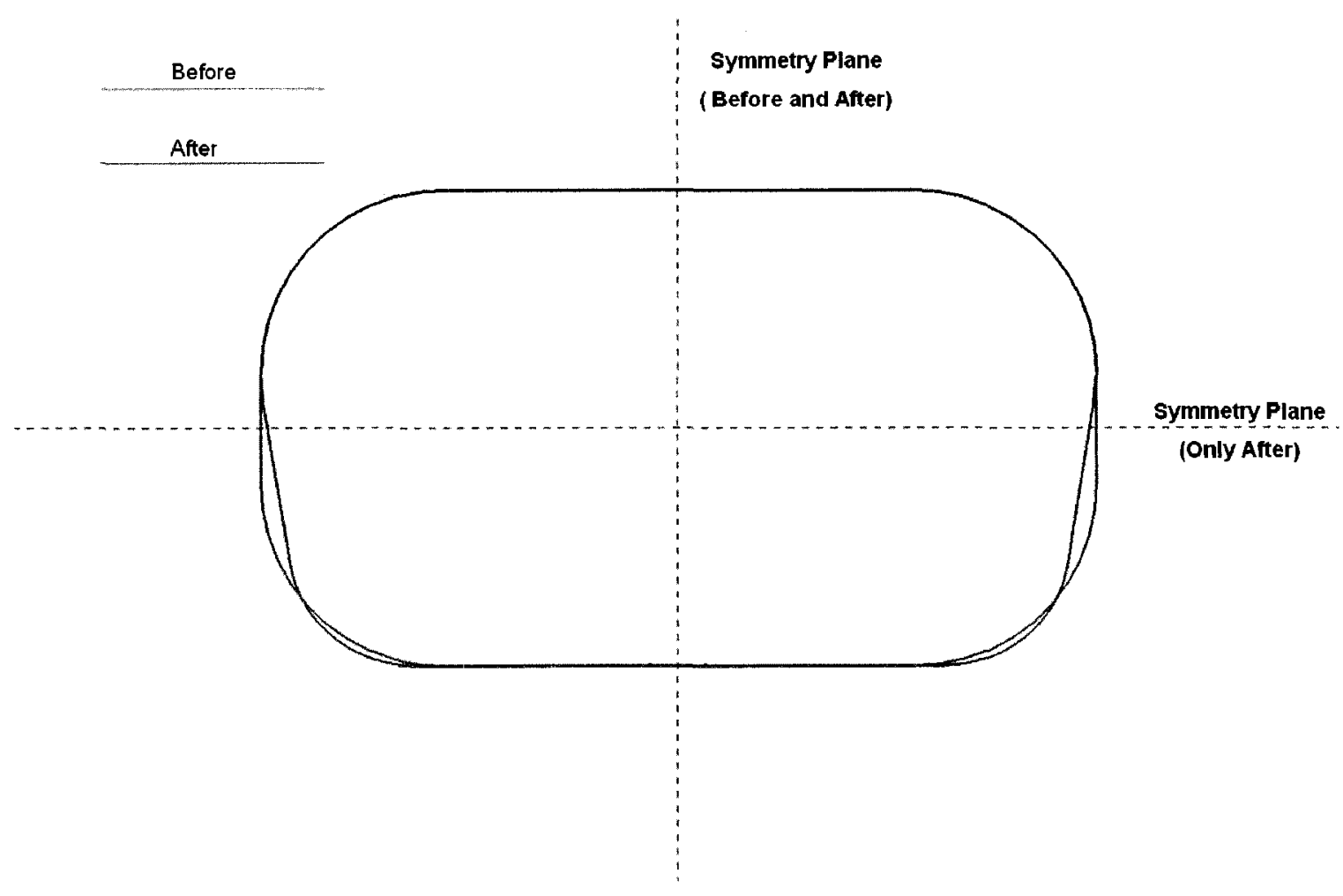

Figure 4-9: Duct Exit Cross-Sectional Shape 


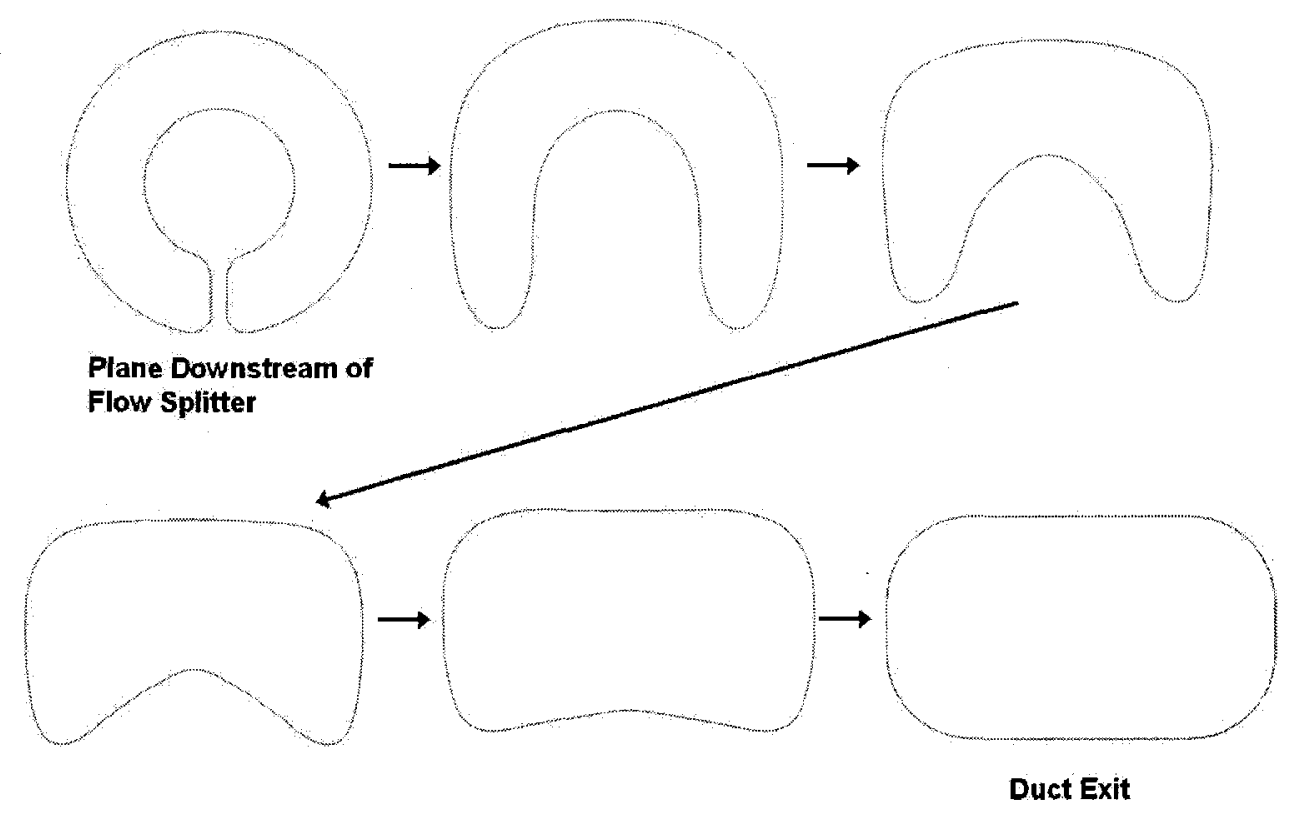

Figure 4-10: Duct Cross-Sections Downstream of Flow Splitter

3. The growth in cross-sectional area should be nearly linear.

4. The exhaust duct geometry should transition smoothly without any waviness or sharp edges that may affect the flow of fluid.

To maintain the wedge angle defined in Sec. 2.2, a cross-section is placed passing through a point marking the end of where the wedge angle is held to. Once the cross-sections are completed, a surface is passed through them and then joined to the upstream flow splitter and annulus, Fig. 4-11.

\subsection{Plenum}

A plenum domain, shown in Fig. 4-12, is created for each duct series as a function of the exhaust stub exit hydraulic diameter. The inlet surface parallel with the stub exit plane has a diameter of $10 D h_{\text {stubexit }}$ and the plenum length is $15 D h_{\text {stubexit }}$. The half cone angle of the plenum is $30^{\circ}$. 


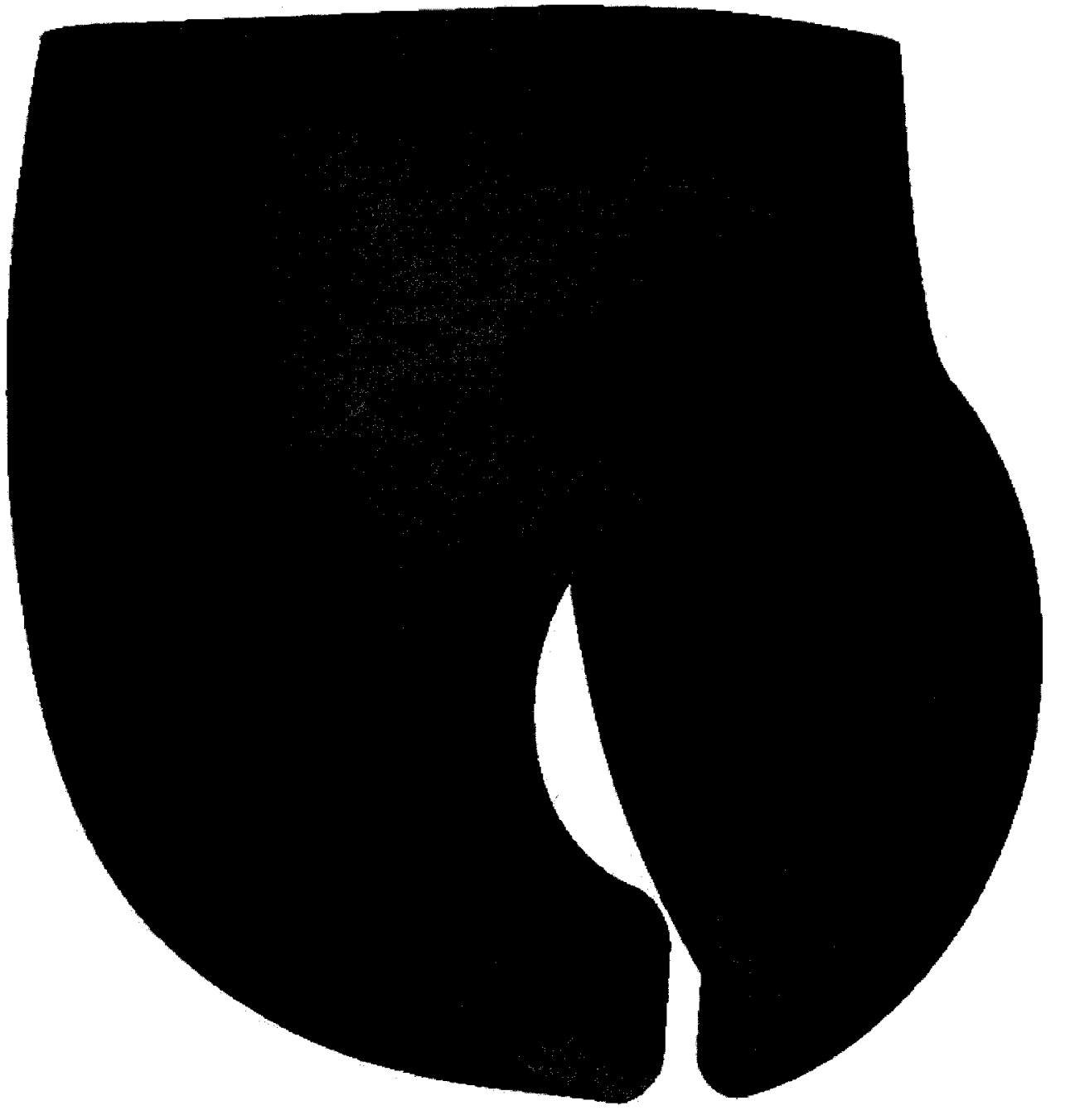

Figure 4-11: Duct Surface Passing Through Cross-Sections 


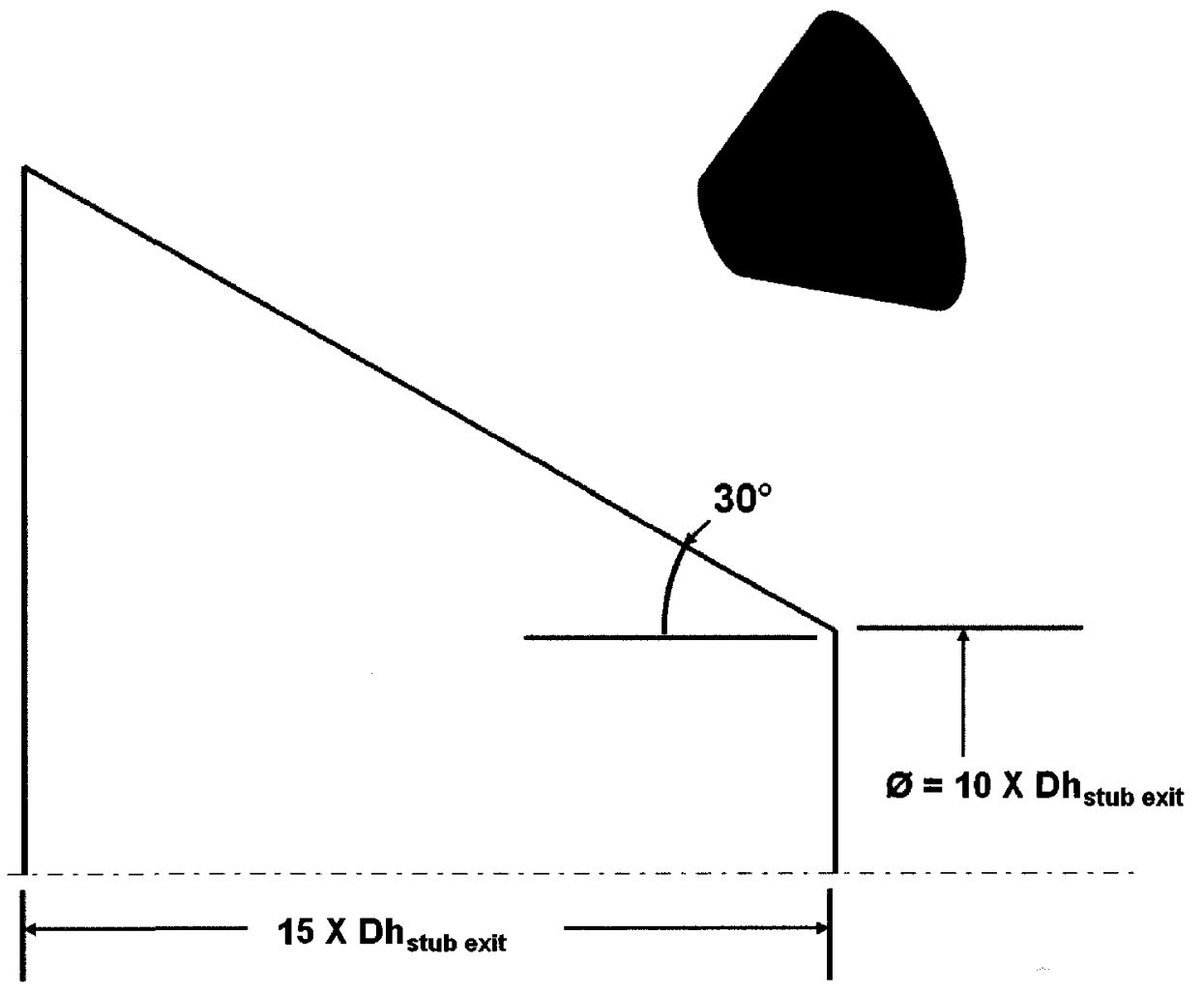

Figure 4-12: Plenum 


\section{Chapter 5}

\section{Computational Study}

The computational analysis has been performed using CFX 5.7.1, which is a commercial Computational Fluids Dynamics (CFD) package developed by ANSYS. CFX 5.7.1, solves the unsteady Navier-Stokes equations in their conservative form. The computational domain is discretized into finite control volumes using a mesh. Each of the governing equations are integrated over a control volume, such that the related quantity (mass, momentum, energy etc.) is conserved in a discrete sense. The following sections cover the development of the computational methodologies and mesh synthesis.

\subsection{Data Reduction}

Data reduction techniques for averaging flow properties must be carefully selected considering the non-uniformity of the flow through the exhaust ducts considered in this study. Errors can be introduced through averaging techniques which will affect the performance parameters introduced in Sec. 2.1, where the total pressure loss coefficient is of prime interest in the current analysis.

Perhaps the simplest form of averaging flow parameters $(\phi)$ is the area average given by:

$$
\bar{\phi}_{\text {area }}=\frac{\int(\phi d A)}{A}
$$

A more apropriate averaging method for non-uniform flows is the mass average method 
given by:

$$
\bar{\phi}=\frac{\int(\phi \rho u d A)}{\int(\rho u d A)}
$$

Wyatt [22] has studied the errors generated using averaging methods that include Eqs. 5.1 and 5.2 in ducts with various velocity profiles. It was demonstrated that total pressure losses in a duct for a Mach number of 0.3 resulted in an error of less then $.1 \%$ and $1 \%$ for the mass average and area average techniques respectively. Based on the above results and to be consistent with the work of Cunningham [8], all flow parameters have been mass averaged in the present study.

\subsection{Pressure-Velocity Coupling}

To overcome the decoupling of pressure and velocity, CFX 5.7.1 uses a single cell, unstaggered, collocated grid. The continuity equation is a second order central difference approximation to the first order derivative in velocity. A fourth derivative in pressure is used to modify the equation to redistribute the influence of pressure and overcome the problem of checker board oscillations found when variables are collocated. The method used is similar to that from Rhie and Chow [23]. A number of extensions are implemented in CFX which improve the robustness of the discretisation when pressure varies rapidly.

\subsection{Advection Scheme}

CFX 5.7.1 offers the first order upwind differencing scheme, the high resolution scheme, or a specified blend factor to blend between first and second order advection schemes to calculate the advection terms in the discrete finite volume equations. The high resolution scheme, used in this study, has a blend factor which varies throughout the domain based on the local flow field. In flow regions where there are low gradients, the blend factor will take on a value close to one representing a second order advection scheme. Flow regions with large gradients will have a blend factor near zero representing a first order advection scheme to prevent overshoots and undershoots and maintain robustness. 


\subsection{Turbulence Modelling}

A wide range of turbulence models are available in CFX 5.7.1 which include a standard $k-\epsilon$, Large-Eddy-Simulation, and the Shear Stress Transport (SST) model. The two most appropriate turbulence models for this analysis are the $k-\epsilon$ and SST turbulence models.

\subsection{1 $\mathbf{k}-\epsilon$}

The $k-\epsilon$ model is robust and computationally inexpensive at solving turbulent flows. The downfall of this turbulence model is that it predicts the onset of flow separation late and tends to under-predict the magnitude of separation. Separated flow has been observed in single port swept exhaust dust by Loka et al [20] and Cunningham [8]. Both authors have observed flow separation along the exhaust duct hub and inner curve in the annular to rectangular transitional region. It is important to note that diffusing flows produce unstable boundary layers due to the flow traveling against an adverse pressure gradient. The effect of predicting the onset of flow separation late results in over predicting exhaust duct efficiencies, where flow separation leads to less pressure recovery and lower discharge coefficients.

\subsubsection{SST}

A turbulence model developed to address the deficiencies of the $k-\epsilon$ model is the SST turbulence model developed by Menter [24]. This model works by solving the $k-\omega$ equations at the near wall region and then the $k-\epsilon$ in the free stream region with a blending function for transition between the two models. The $k-\omega$ based SST model takes into account the transport of the turbulent shear stress to give highly accurate predictions of the onset and the amount of flow separation under adverse pressure gradients. The key reason for the deficiencies of the $k-\epsilon$ model is that is does not account for the transport of the turbulent shear stress resulting in an over prediction of the eddy-viscosity.

CFX guidelines for using this model requires an overall $y+$ of less then two and no less then 10-15 grid points within the boundary layer. The SST model continues to be accurate when these guidelines can not be achieved from the use of scalable wall functions. For fine 


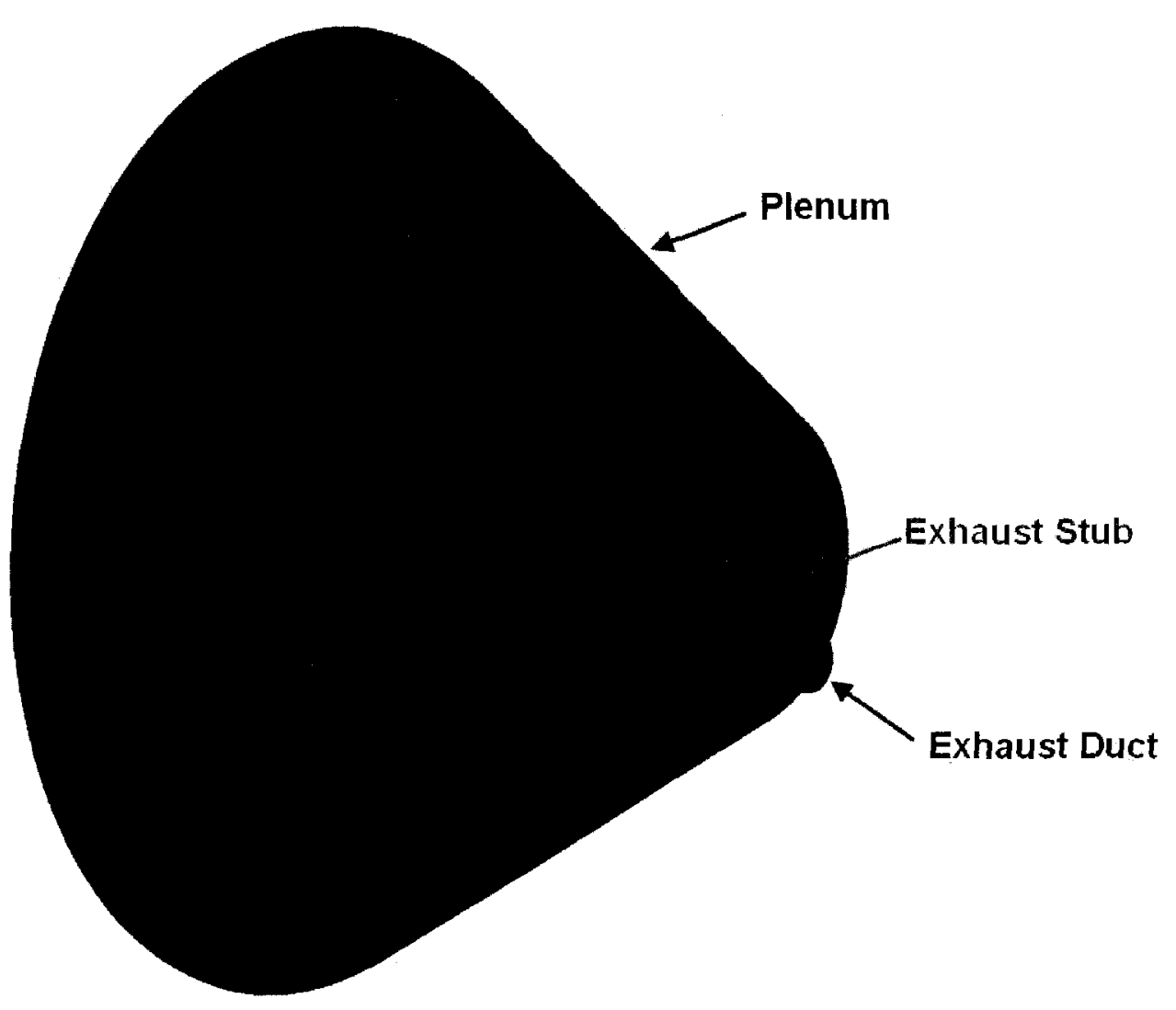

Figure 5-1: Air solid model of the computational domain

grids the $k-\omega$ model is applied, but when a coarser grid is used, the model switches to a wall function treatment making use of the logarithmic profile assumptions. For this reason, a new near wall treatment was developed by CFX for the $k-\omega$ based models that allows for a smooth shift from a low-Reynolds number form to a wall function formulation.

\subsection{Computational Domain}

The computational domains studied in this work are composed of an exhaust duct, exhaust stub, and a plenum chamber as illustrated in Fig. 5-1. The plenum chamber has been modeled to serve multiple functions which include providing a far-field boundary condition and a domain for jet flow entrainment. The work of Loka et al, and Cunnigham have made use of plenum chambers in their work, both demonstrating that non-uniform pressure 
gradients exist at the exhaust stub exit. These pressure gradients are found to exist as a result of the swirling flow and geometric influences causing streamline curvature. If no plenum is modeled, the flow structure at the exhaust stub exit would be strongly influenced by the imposed boundary conditions. A realistic exit flow structure can only then be achieved if the pressure gradients at the exhaust stub exit are known in advance.

\subsubsection{Boundary Conditions}

The CFD boundary conditions have been selected as being the most appropriate for determining the aerodynamic and geometric parameters sensitivity on exhaust duct losses. The boundary conditions are not consistent with what is experienced at flight conditions where ambient conditions change according to aircraft speed, altitude, and engine installation. The following boundary conditions can be seen in Fig. 5-2.

\section{Duct Inlet}

Ansys CFX contains a large variety of inlet boundary conditions to suit many situations. The requirements of this study are to impose a swirl gradient and aerodynamic blockage at the duct inlet plane. Total pressure and mass flow boundary condition do allow for an imposed flow direction, however, the aerodynamic blockage is an implicit result of the flow simulation making these boundary conditions a poor choice for the current study. The most suitable inlet boundary condition for this study, that is offered in CFX, is to impose a velocity gradient with magnitude and direction. Using this option both a swirl gradient and blockage can be controled explicitly. Using the later choice of inlet boundary conditions, the velocity magnitude and direction were specified as a function only of radius (constant across the circumference). An inlet total temperature of $1530 \mathrm{R}$ was used throughout this study, being a common average total temperature for P\&WC swept exhaust ducts. The final parameters set at the inlet to the exhaust duct are turbulence intensity and eddy viscosity ratio. A turbulence intensity of $10 \%$ and an eddy viscosity ratio of 300 were suggested by P\&WC experts and used throughout the study. 


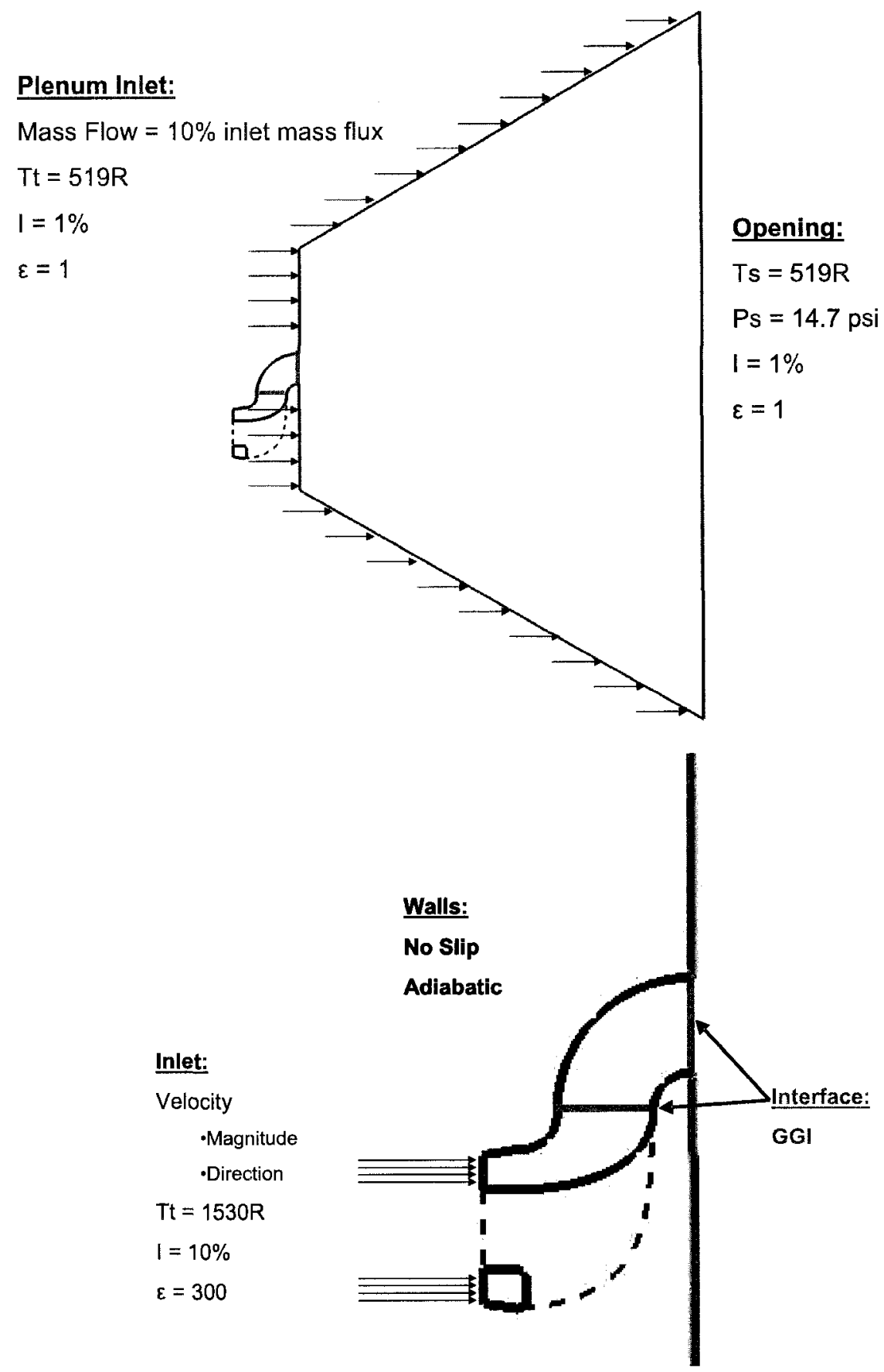

Figure 5-2: CFD boundary conditions 


\section{Plenum}

The conical plenum domain has an inlet and an outlet boundary condition. The inlet boundary condition is mass flow with direction. The mass flow rate is implicitly defined to be $10 \%$ of the duct inlet mass flux. The flow direction over the full surface is directed normal to the exhaust stub exit plane. A uniform total temperature is imposed at 519 R. A low inlet turbulence intensity and eddy viscosity ratio of $1 \%$ and 1 respectively was used. The plenum exit was modeled as an opening allowing both inflow and outflow from the same location. The opening static pressure and temperature is set at $14.7 \mathrm{psi}$ and 519 $R$ respectively. For the condition of inflow through the opening, a low turbulence intensity and eddy viscosity ratio was selected to match the plenum inlet boundary condition.

\section{Walls}

The exhaust duct and stub wall have been modeled as no slip and adiabatic.

\subsubsection{Grid Structure}

The computational grid was created using ANSYS ICEM CFD. This meshing package offers the capability to create grids in multi-block structured, unstructured hexahedral, tetrahedral, hybrid grids consisting of hexahedral, tetrahedral, pyramidal and prismatic cells. For this study, the computational domain was constructed using unstructured tetrahedral elements with prismatic cells for near wall turbulence model requirements.

The three components of the computational domain (exhaust duct, stub, and plenum) where meshed separately and later assembled in CFX making use of General Grid Interface (GGI) which is used to create a fluid-to-fluid interface layer between two grid surfaces which do not have matching node locations. The GGI theory involves a control surface treatment of the numerical fluxes across the interface. The handling of the interface fluxes is fully implicit and fully conservative in mass, momentum, and energy. The advantage of using GGI capability of ANSYS CFX, is that a parametric study can be carried out with minimal time spent creating computational grids. For one duct series in Chapter 3, only one exhaust duct, one curved stub, one straight stub, and one plenum is needed to be meshed in ICEM CFD. The C-Shaped domain is created by assembling the exhaust duct, curved stub, and 


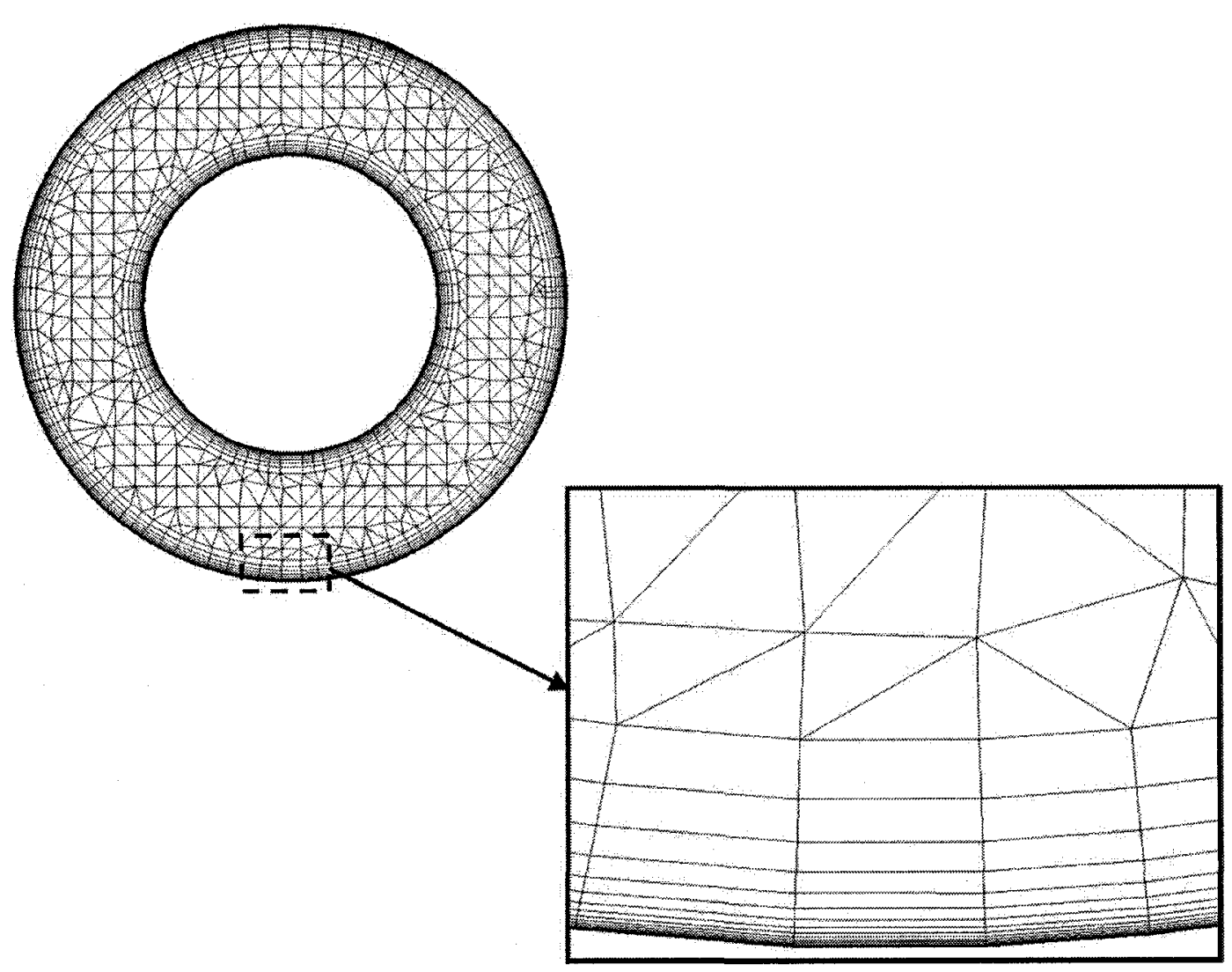

Figure 5-3: Exhaust Duct Inlet Showing Prism Layer Elements (20 layers shown)

plenum in ANSYS CFX and defining all the fluid-to-fluid interface surfaces. The S-Shaped duct is created in ANSYS CFX by rotating the stub and plenum about an axis. To create the Intermediate-Shaped duct, the curved stub is swapped with the straight stub, and the plenum is rotated and translated in space to match interface surfaces.

\section{Exhaust Duct and Stub Grid}

The exhaust duct and stub grids are composed of prism elements to resolve the boundary layer and tetrahedral elements to resolve the free-stream flow. These two computational domains must respect the mesh requirements of the SST turbulence model discussed in Sec. 5.4.2 since the walls in these domains are defined in CFX as no-slip surfaces where a boundary layer is present. Prism elements are inflated from the walls of each domain up to 


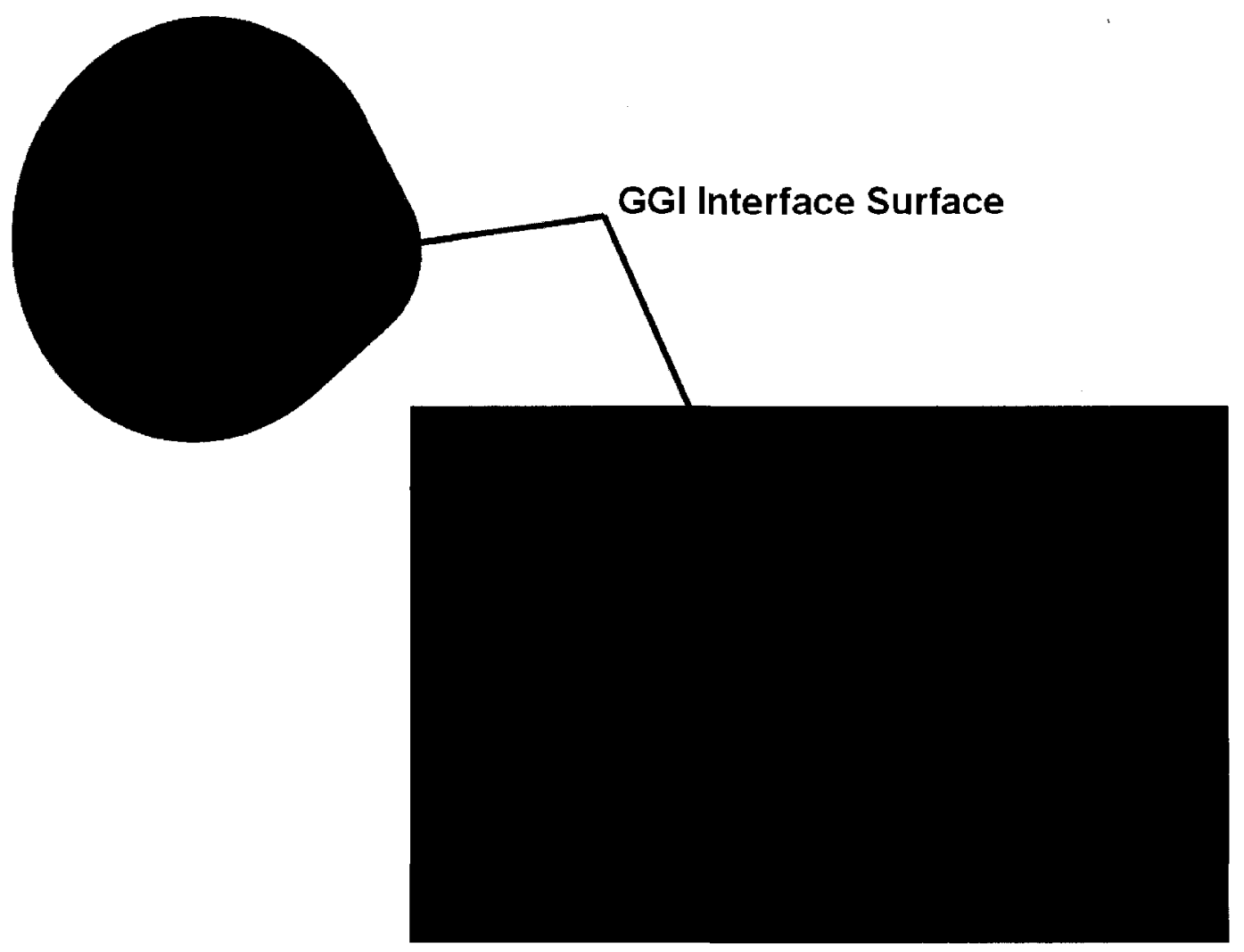

Figure 5-4: Plenum surface grid

a height which meets minimum requirement of putting 10-15 nodes in the boundary layer, see Fig. 5-3. With the inlet area of the exhaust duct fixed in this study the prism layers have been extended to a height of $h / 6$ measured normal from the wall surfaces. The prism elements are formed by first growing one layer to the overall inflation layer height, $x_{n}$, then subdividing them using an exponential growth law with a height ratio of $d$, giving a smooth transition of the last prism layer height, $x_{n-1}$, to the tetrahedral elements resolving the free stream flow. The height of a given prism layer, $i$, can be calculated from Eq. 5.3.

$$
x_{i}=x_{1} \times d^{i-1}
$$




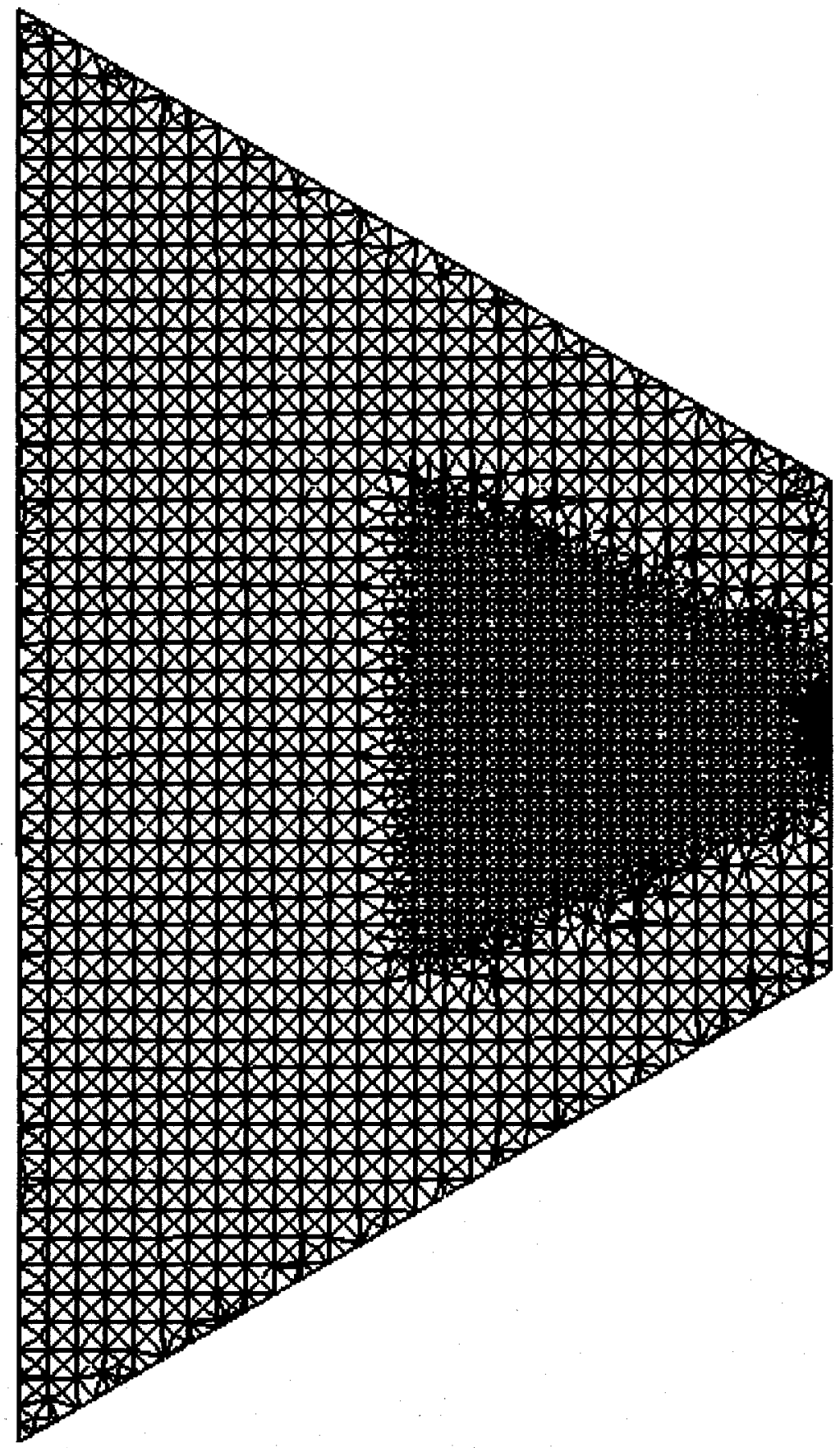

Figure 5-5: Cross-section of the plenum domain showing element sizes 


\section{Plenum Grid}

The plenum domain is meshed with the inlet and opening surfaces as was explained in Sec. 5.5.1. Only tetrahedral elements are used in this domain because there are no noslip surfaces requiring the grid resolution needed to resolve the boundary layer. One more surface is included in this domain, shown in Fig. 5-4, having the same cross-sectional shape as the exhaust stub exit plane. This surface is included for the GGI interface boundary condition used to create the fluid-to-fluid interface which links the flow from the exhaust stub to the plenum. The elements on this surface are sized to match the exhaust stub tetrahedral size. The remaining surfaces do not require fine elements because high gradient are not expected in these regions. The elements on these remaining surfaces are created eight times larger then what is used in the exhaust duct and stub grid. Two ease the transition from the small elements in the exhaust stub domains to the large elements in the plenum, a volume of elements four times larger then the exhaust stub are produced in the region where the jet flow first enters the plenum, Fig. 5-5.

\subsubsection{Grid Study}

Prior to fixing the mesh parameters for the current study, a sensitivity analysis has been preformed to select the appropriate quantity of prism layers and tetrahedral grid density.

\section{Selecting the Quantity of Prism Layers}

Three sets of exhaust duct and stub meshes were created to test the sensitivity of the total to total pressure losses versus the quantity of prism layers within an overall prism layer height of $h / 6$. For the three sets of meshes, total prism layer quantities of $n=20,25$, and 30 were studied while the height, $x_{1}$, of the first node from the wall was maintained constant. Each of the meshes was created using the same density of tetrahedral elements and same CFD boundary conditions so as to isolate the differences in numerical solution with respect to the quantity of prism layers. The results of this analysis are plotted in Fig. 5-6. It is apparent that increasing the number of prism layers has only a small effect on the total pressure losses within the exhaust duct and stub. Total pressure losses varied by only $2 \%$ when going from 20 to 30 prism layer. Considering the small differences in pressure 


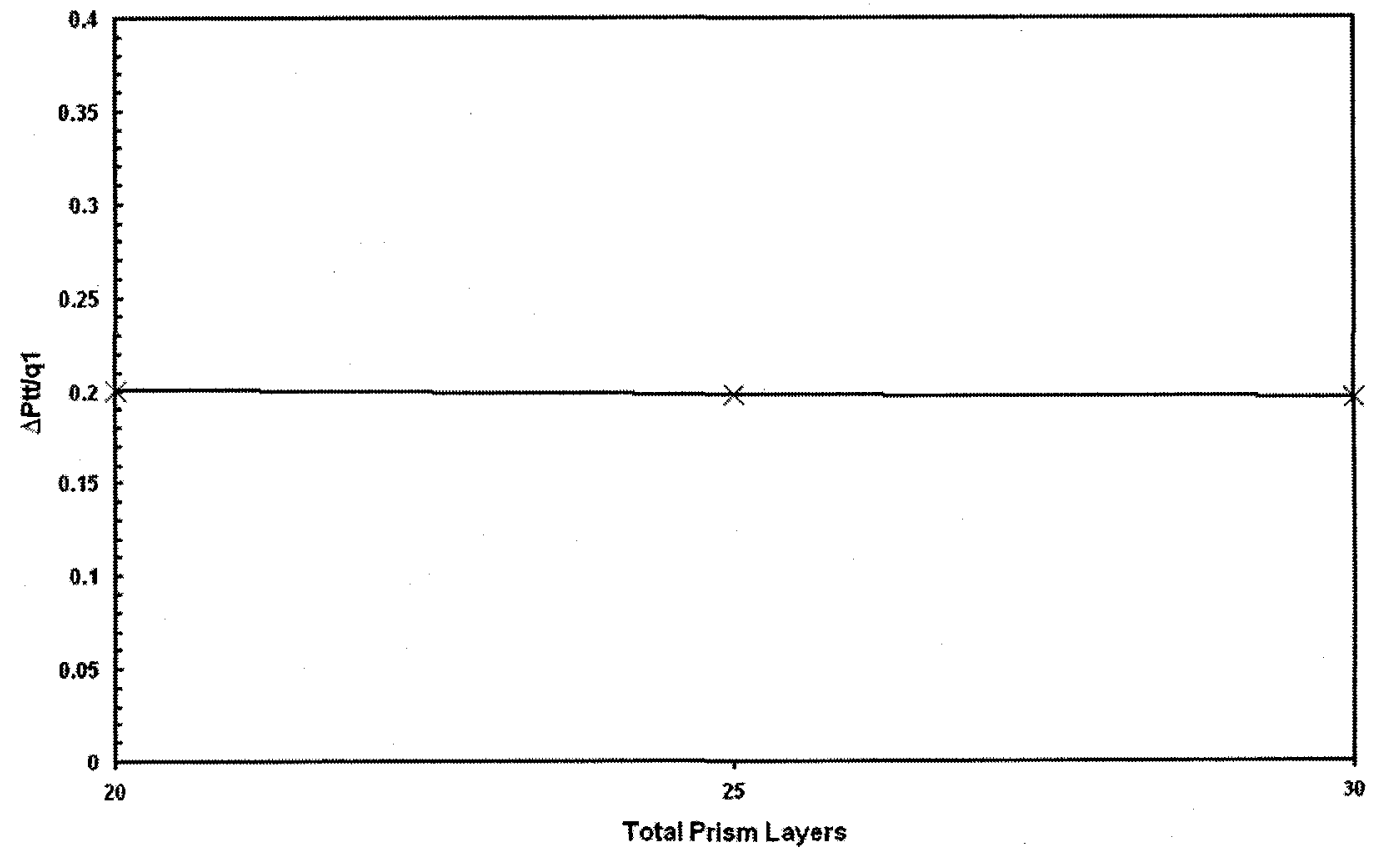

Figure 5-6: $\frac{\Delta P_{t t}}{q_{1}}$ ploted versus of total number of prism layers 

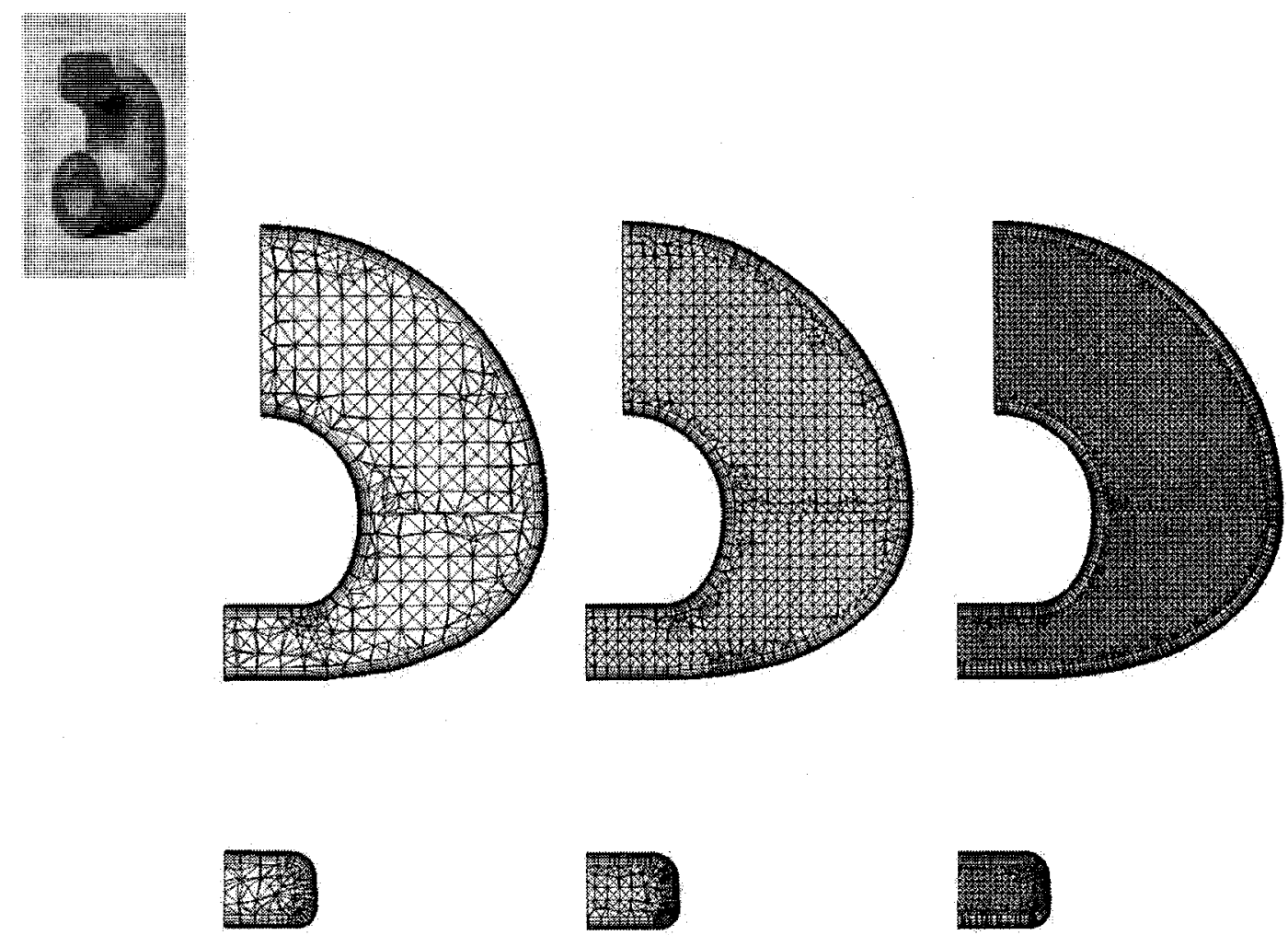

Coarse

Intermediate

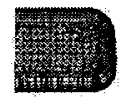

\section{Fine}

Figure 5-7: Three grids constructed from tetrahedral sizes of $h / 3$ (coarse), $h / 6$ (intermediate), and $h / 12$ (fine)

losses, 20 prism layers have been used thought this study resulting in a $25 \%$ reduction in nodes versus the 30 prism layer runs.

\section{Selecting the Grid Density}

Grid dependence on grid density has been examined by constructing computational domains using different tetrahedral element sizes. Three domains were created using tetrahedral sizes of $h / 3, h / 6$, and $h / 12$ while maintaining the same prism parameters as selected in Sec. 5.5.3. Figure 5-7 demonstrates that the selected tetrahedral parameters lead to a coarse mesh of 135465 nodes, an intermediate mesh of 322371 nodes, and a fine mesh of 980284 nodes. The three grids were solved numerically using the same boundary condition used in Sec. 5.5.3 and 


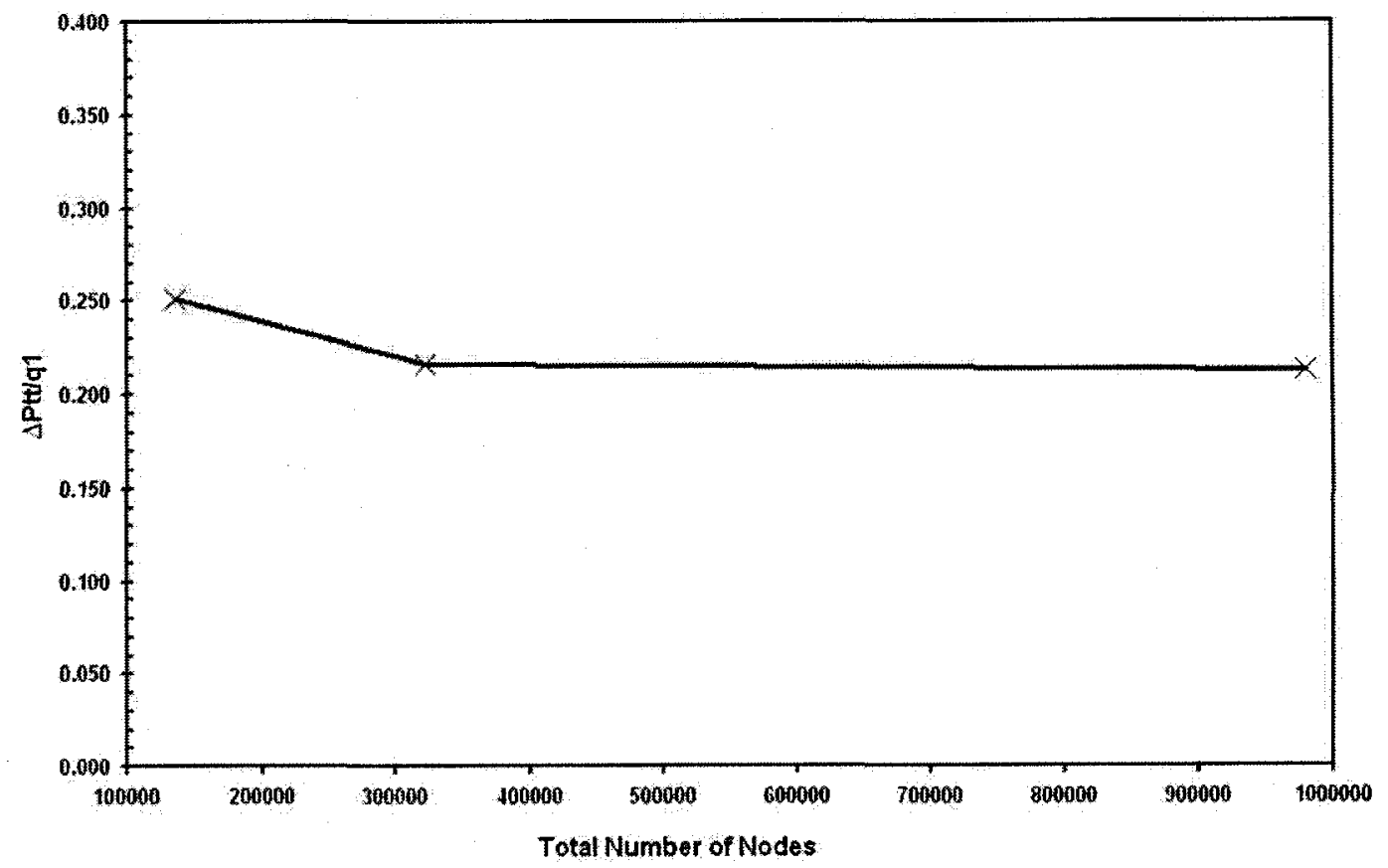

Figure 5-8: $\frac{\Delta P_{t t}}{q_{1}}$ ploted versus of total number nodes 

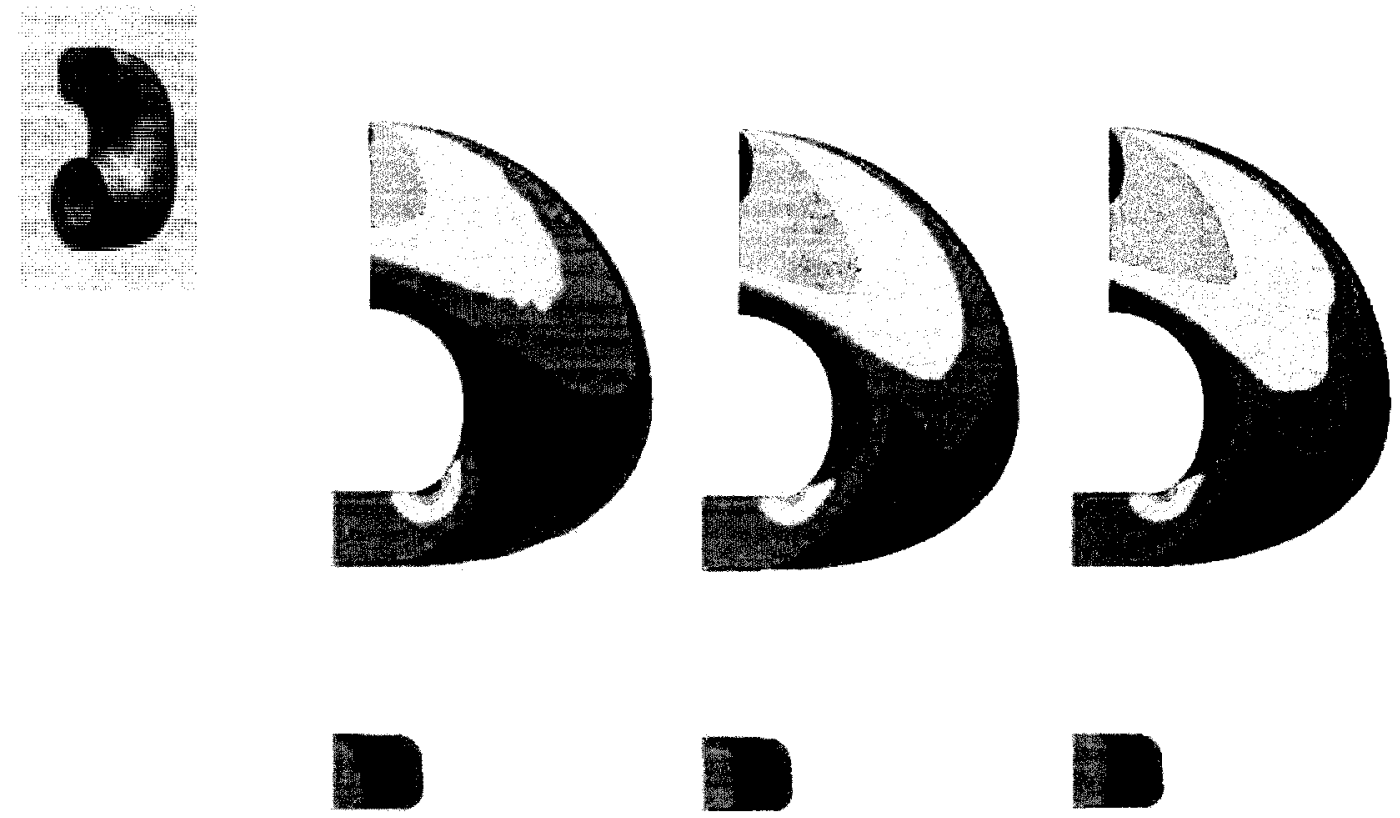

\section{Coarse}

Intermediate

Fine

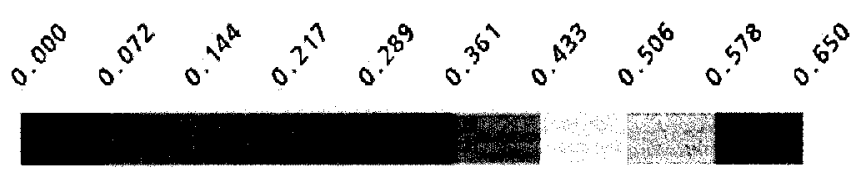

Mach Number

Figure 5-9: Mach contours for the three grid densities 
the resulting total pressure losses are plotted in Fig. 5-8. It is apparent that grid dependence is obtained with a difference in total pressure losses of $1.4 \%$ between the intermediate and fine grids. Figure 5-9 further demonstrates that grid dependence is obtained by viewing the Mach contours on the symmetry plane of the three grids. Examination of Fig. 5-9 shows that the coarse grid fails to pick up the flow separation in the exhaust duct and stub along the inner bend which is present in the other grids. Figure 5-9 also demonstrates that a lower magnitude of flow separation along the exhaust duct center body is obtained in the coarse grid where the intermediate and fine grids both demonstrate that flow is separating at the same order of magnitude. Based on these results the remainding grids in the current study have been created using a maximum tetrahedral size of $h / 6$ with a computation savings of nearly $70 \%$ less nodes than what was used in the fine grid. 


\section{Chapter 6}

\section{CFD-Based Parametric Study}

The following sections present the findings of CFD analysis. Each geometric and aerodynamic parameter is presented and it is demonstrated, qualitatively and quantitative, how they are related to the total pressure loss in the exhaust duct. Cross-sections are defined throughout the study according to the method discussed in Chapter 4 to evaluate and compare the losses between each duct studied. The sections presented in Fig. 6-1 will be referenced throughout this Chapter, and distance is calculated along the gas path spline.

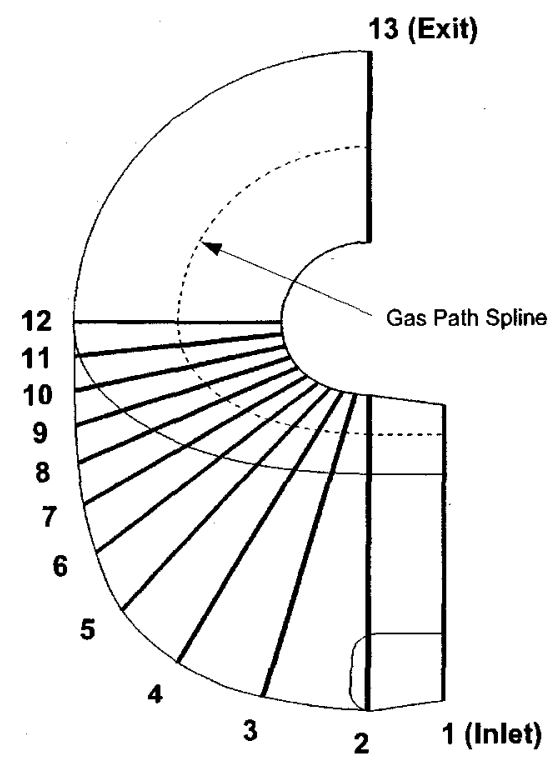

Figure 6-1: Cross-sections used for data reduction and flow visualization 


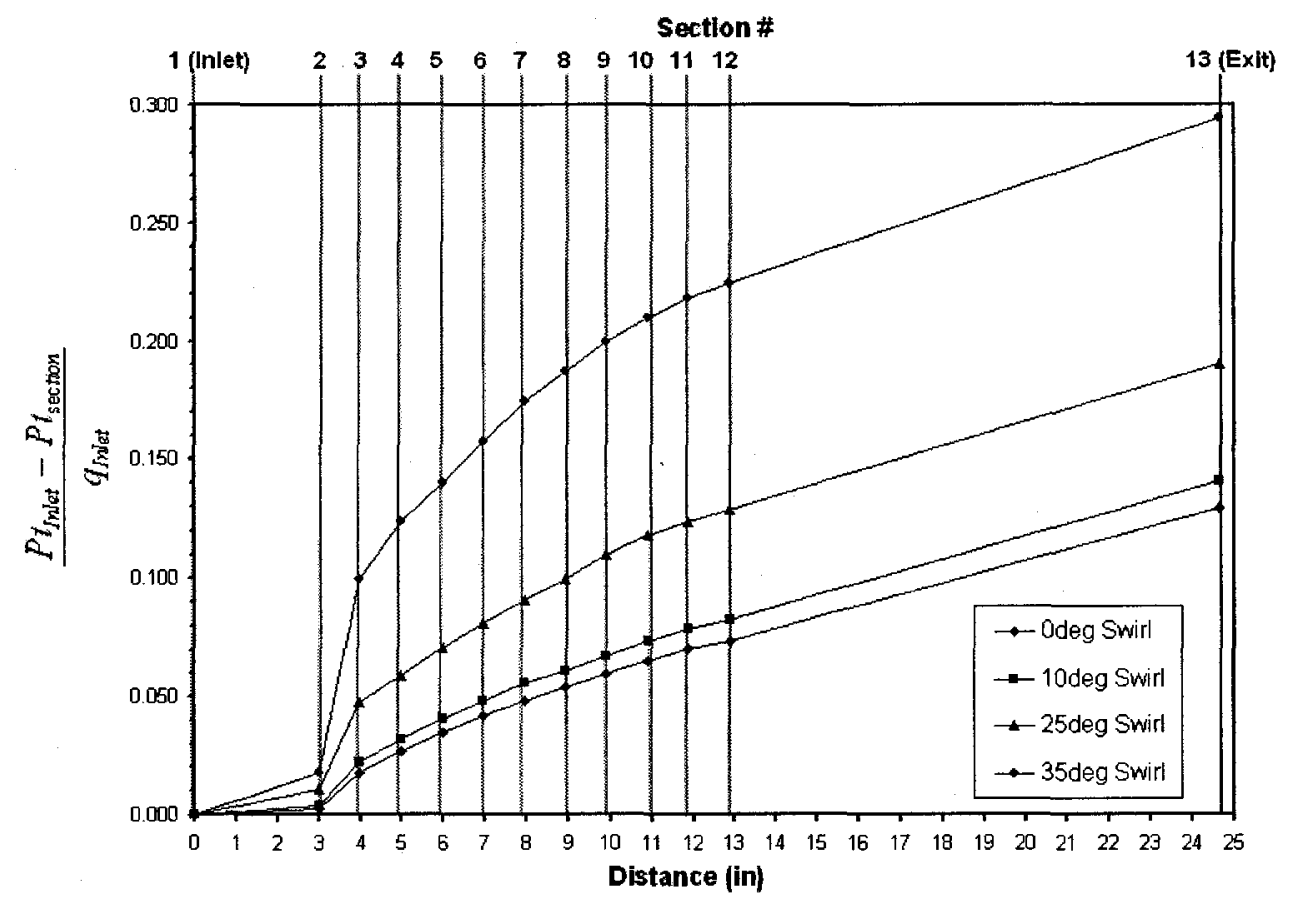

Figure 6-2: Total pressure loss coefficient evaluated at each section defined in figure 6-1

\subsection{Effect of Swirl}

Inlet swirl has been demonstrated to have a large influence on the internal flow structure producing higher losses as inlet swirl is increased. As indicated in Fig. 6-2, the losses are at a minimum for nominal $0^{\circ}$ inlet swirl for a sample exhaust duct with a $\mathrm{C}$-shaped stub. In this figure, the total pressure loss coefficient is calculated at each section from Fig. 6-1 demonstrating how the total pressure losses can be seen to accumulate. To illustrate this, contours of the normal component of velocity with vectors of the tangential component is plotted on cross-sections for the sample exhaust duct in Figs. 6-3 and 6-4 for inlet boundary conditions of nominal $0^{\circ}$ and $35^{\circ}$ inlet swirl with low inlet blockage.

The increasing loss between the inlet and section 2 can be attributed to the higher velocity gradients. A symmetric flow field can be seen at section 2 for inlet swirl of nominal $0^{\circ}$ where when inlet swirl is higher as in the case of nominal $35^{\circ}$ the flow field is asymmetric. This asymmetry in flow is caused as a result of an upstream influence of the flow splitter. The resulting flow field has larger velocity gradients at higher inlet swirl conditions where 

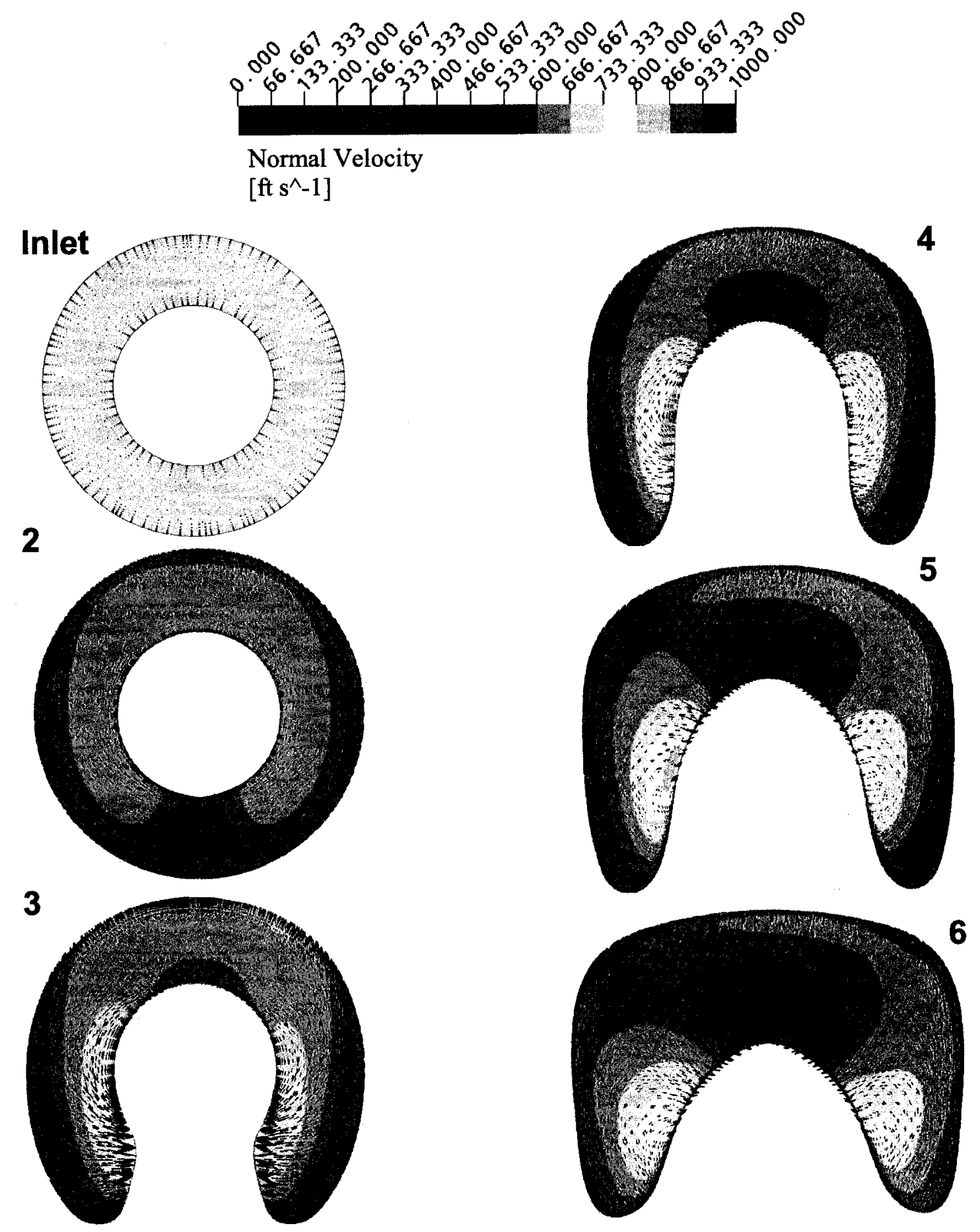

Figure 6-3: Velocity contours normal to cross-section (nominal $0^{0}$ swirl) 

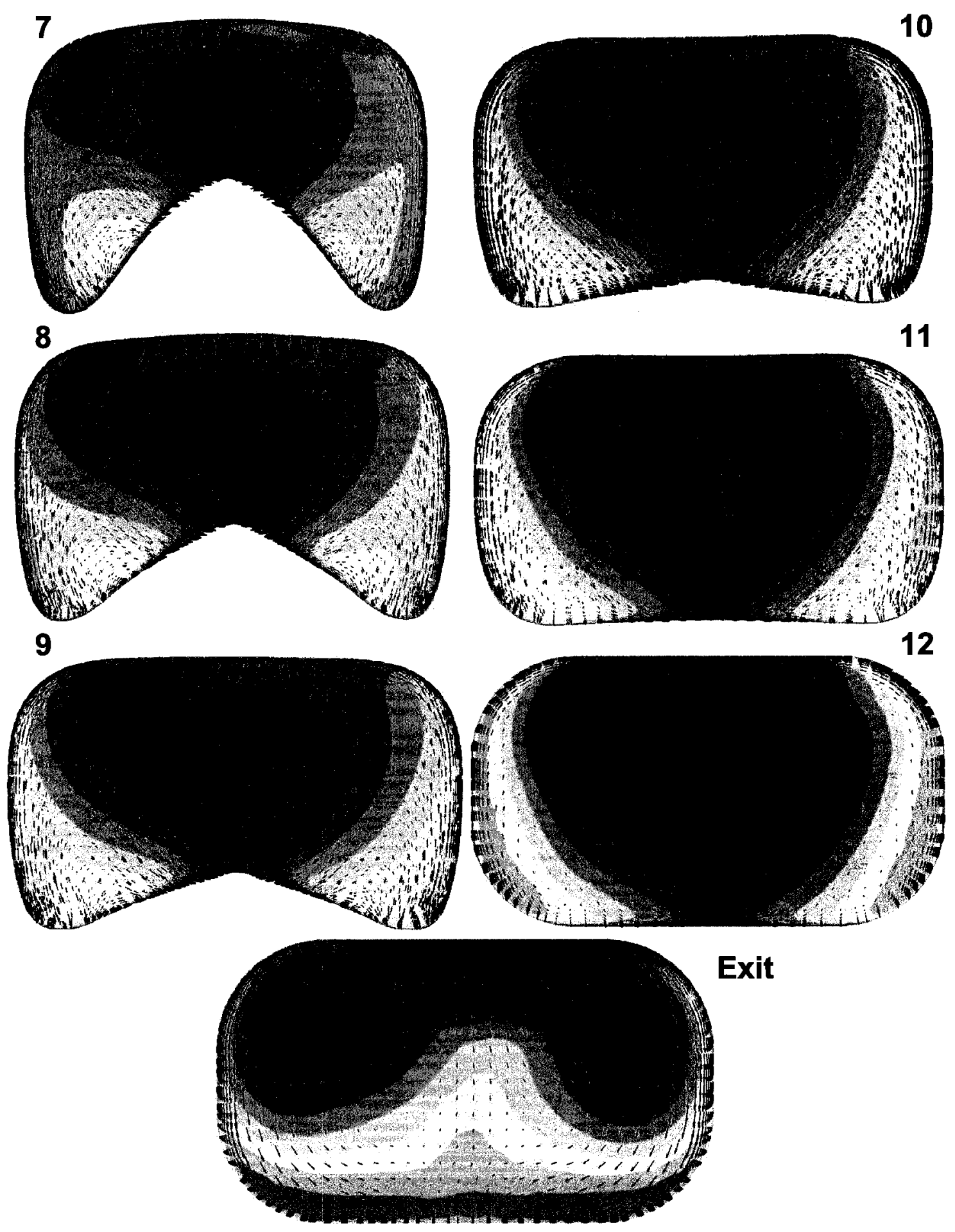

Figure 6-3: Velocity contours normal to cross-section (nominal $0^{0}$ swirl)....con't 

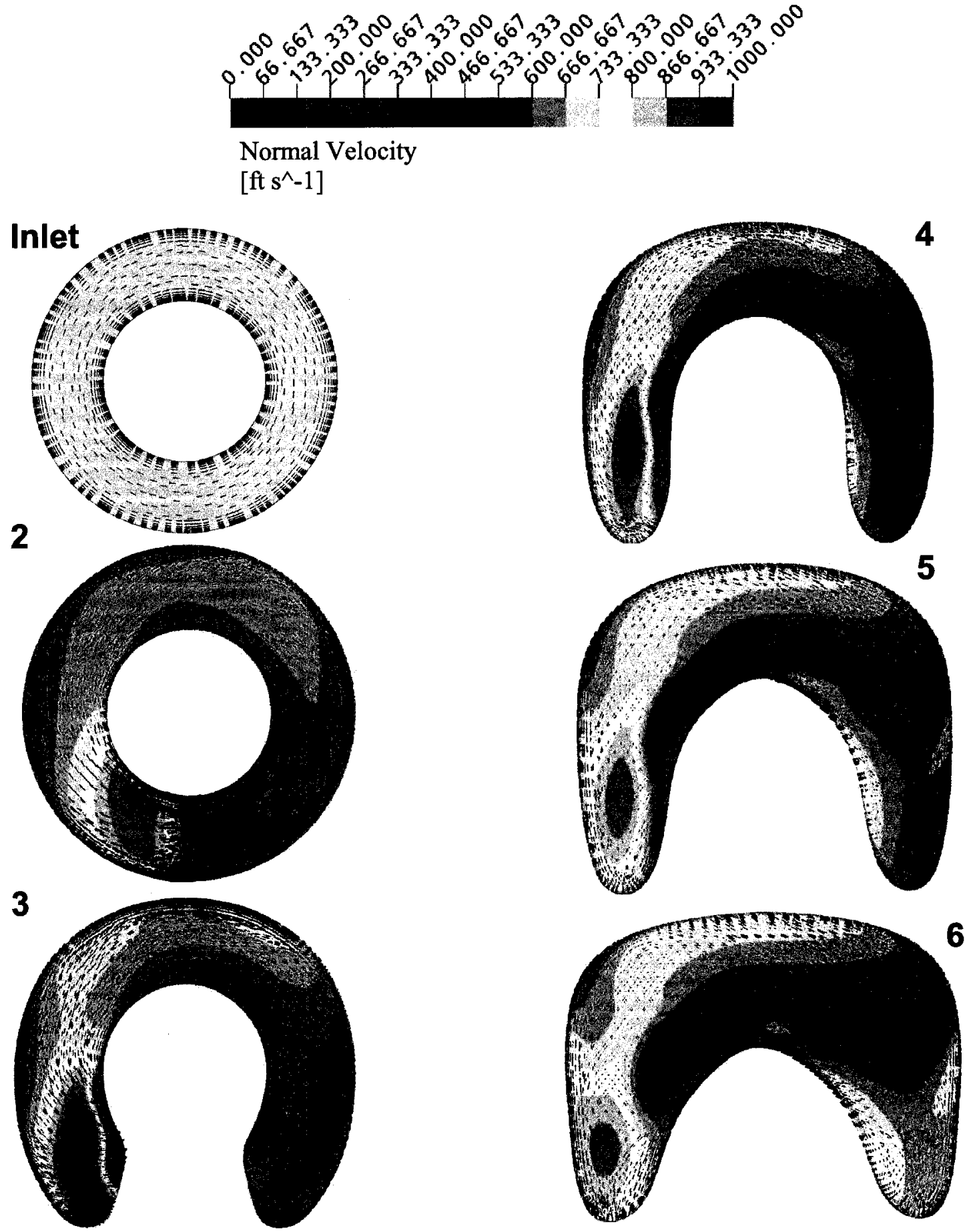

Figure 6-4: Velocity contours normal to cross-section (nominal $35^{0}$ swirl) 

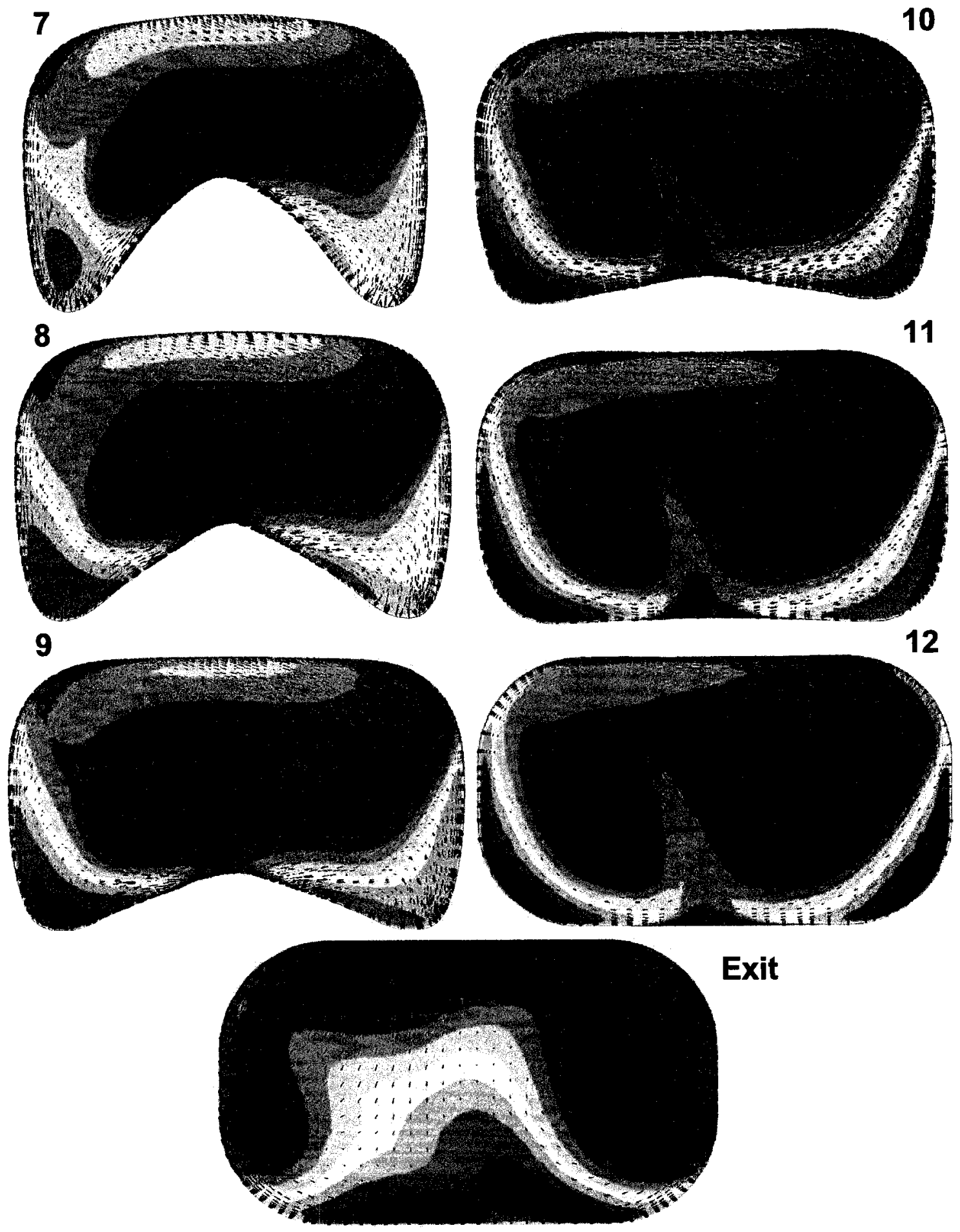

Figure 6-4: Velocity contours normal to cross-section (nominal $35^{0}$ swirl)....con't 
viscous shear produces increasing pressure losses.

A large increase in losses is always found to occur between sections 2 and 3 . It is at these sections that the exhaust duct begins transitioning from annular to rectangular and is where gradients in velocity are highest. Observing Figs. 6-3 and 6-4 at section 3 shows that inlet swirl transforms the flow field and introduce regions of peak velocity and flow separation due to incidence angles with the flow splitter. The losses rise at section 3 with increasing swirl due to increasing asymmetry in the flow field and increasing flow separation.

Sections 3 through 12 finishes the exhaust duct transition from annular to rectangular, and ends at section 12 bringing the flow out in a direction $90^{\circ}$ from the inlet. The total pressure losses through these sections are found to increase steadily for all inlet swirl conditions as indicated in Fig. 6-2. In Fig. 6-3 for an inlet swirl of nominal $0^{\circ}$ high velocity flow remains concentrated in the lower portion of section 3 near the hub and then separates off the centerboby at section 5 in a manner similar to Von Karman Vortex shedding. This low velocity flow can be seen to penetrate in to the core flow field, and is dissipated by section 12. Counter rotating vortices are seen to form in the core flow by section 8 due to the fluid turning while the low momentum fluid in the boundary layers at the outer sides of the section is seen moving opposite to the vortices due to low pressure at the inner surface of the bend. For the same sections with inlet swirl as in Fig. 6-4, the flow field is more complex. At section 3 the flow is already seen to be separating at the hub and is joined at section 4 with the separated flow off the flow splitter. High momentum flow is seen concentrated along the left hand side of sections 3 through 9 until it becomes fairly more uniform by section 10 . The low velocity pocket of separated flow observed at section 4 is seen to leave the centerbody at section 5 and can be seen to be almost fully dissipated by section 12. The effect of turning the flow $90^{\circ}$ is again shown by the presence of two counter rotating vortices which are almost fully developed by section 9 .

The remaining duct losses are occurring through the exhaust stub between section 12 through 13 where in both Figs. 6-3 and 6-4 display flow separation along the inner surface due to the momentum of the fluid being pressed along the outer surface in the remaining $90^{\circ}$ turn. The addition of inlet swirl produces a exhaust stub exit flow structure slightly asymmetric to what is observed with nominal $0^{\circ}$ inlet swirl. 


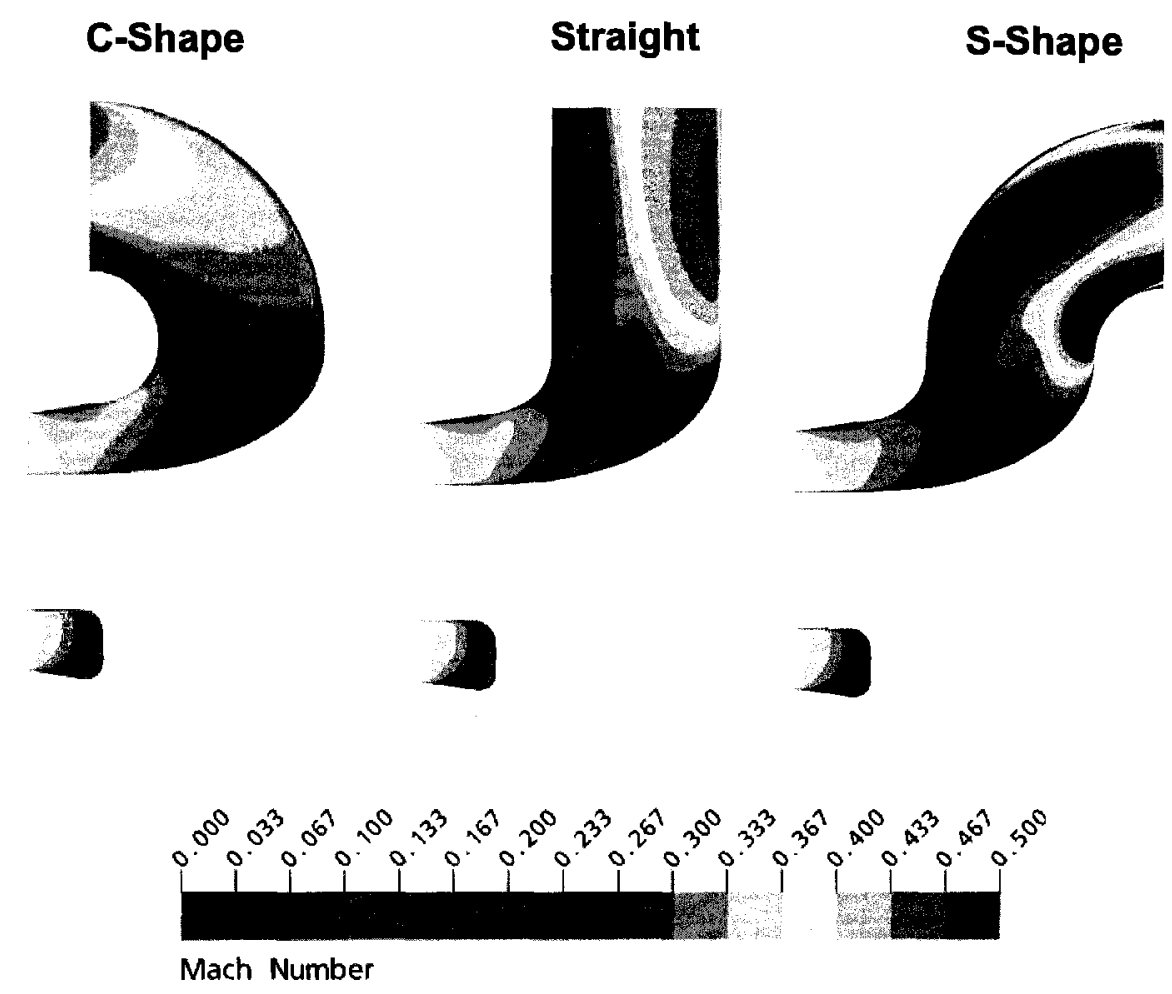

Figure 6-5: Mach contours at mid plane demonstrating the effect of stub direction (nominal $0^{0}$ inlet swirl)

\subsection{Effect of Stub Direction}

The exhaust stub direction has been shown to have an impact on the internal flow structure in the exhaust duct. Figure 6-5 demonstrates through Mach contours the effect of stub direction at nominal $0^{\circ}$ inlet swirl on the internal flow structure. At the nominal inlet swirl of $0^{\circ}$ there is more flow separation in the case of the Straight stub and the S-shaped stub then what is found in the C-shaped stub. The reason for this is seen in Fig. 6-6 where static pressure contours are presented. In the case of the C-shaped stub, a high pressure region is present along the outer curvature of the duct which prevents flow separation along the inner curvature by forcing the flow to follow the surface. As for the case of the Straight and S-shape stubs, this high pressure region is not present to prevent flow separation as seen in Fig. 6-6. 


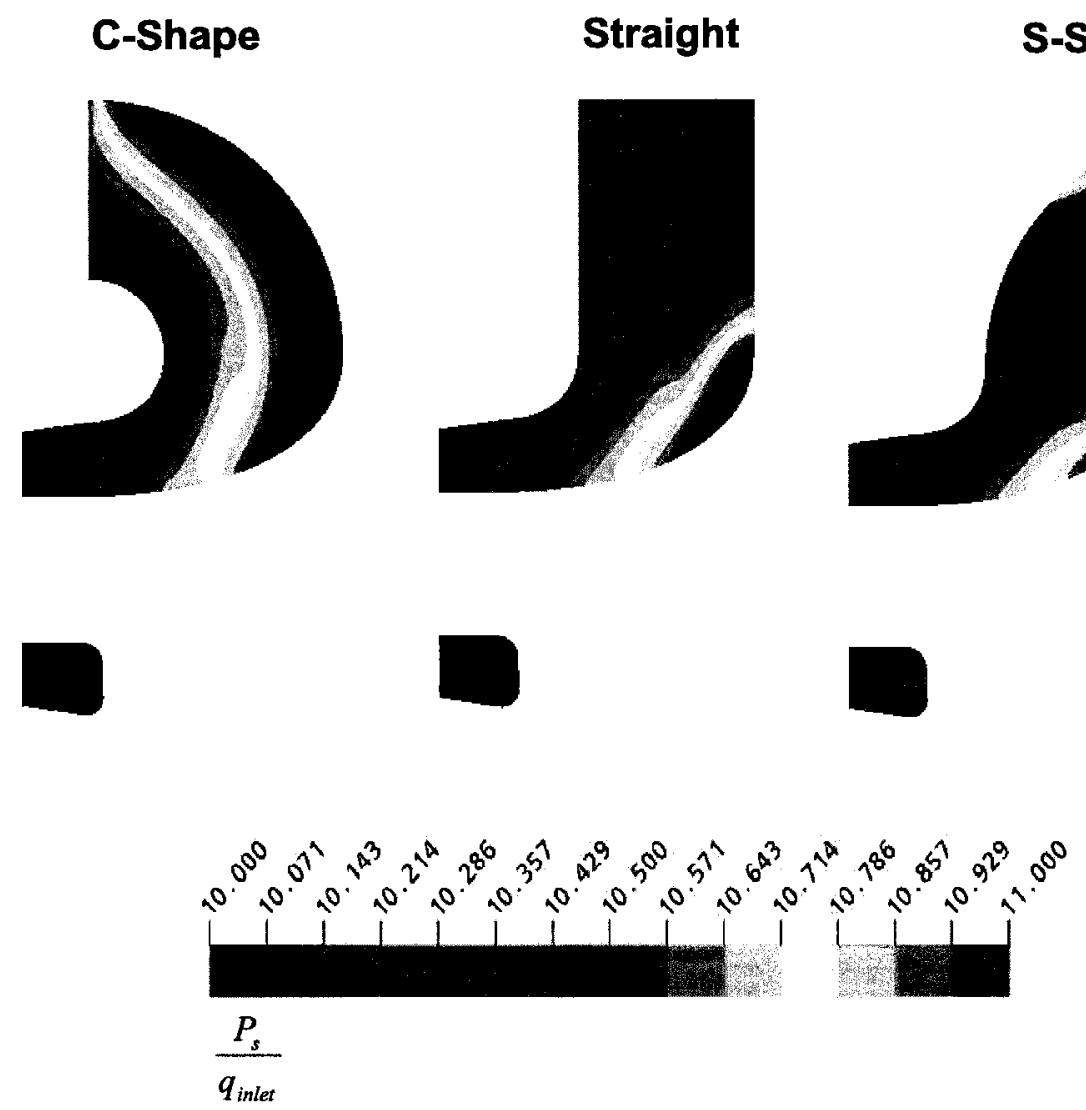

Figure 6-6: Normalized static pressure contours at mid plane demonstrating the effect of stub direction (nominal $0^{0}$ inlet swirl) 


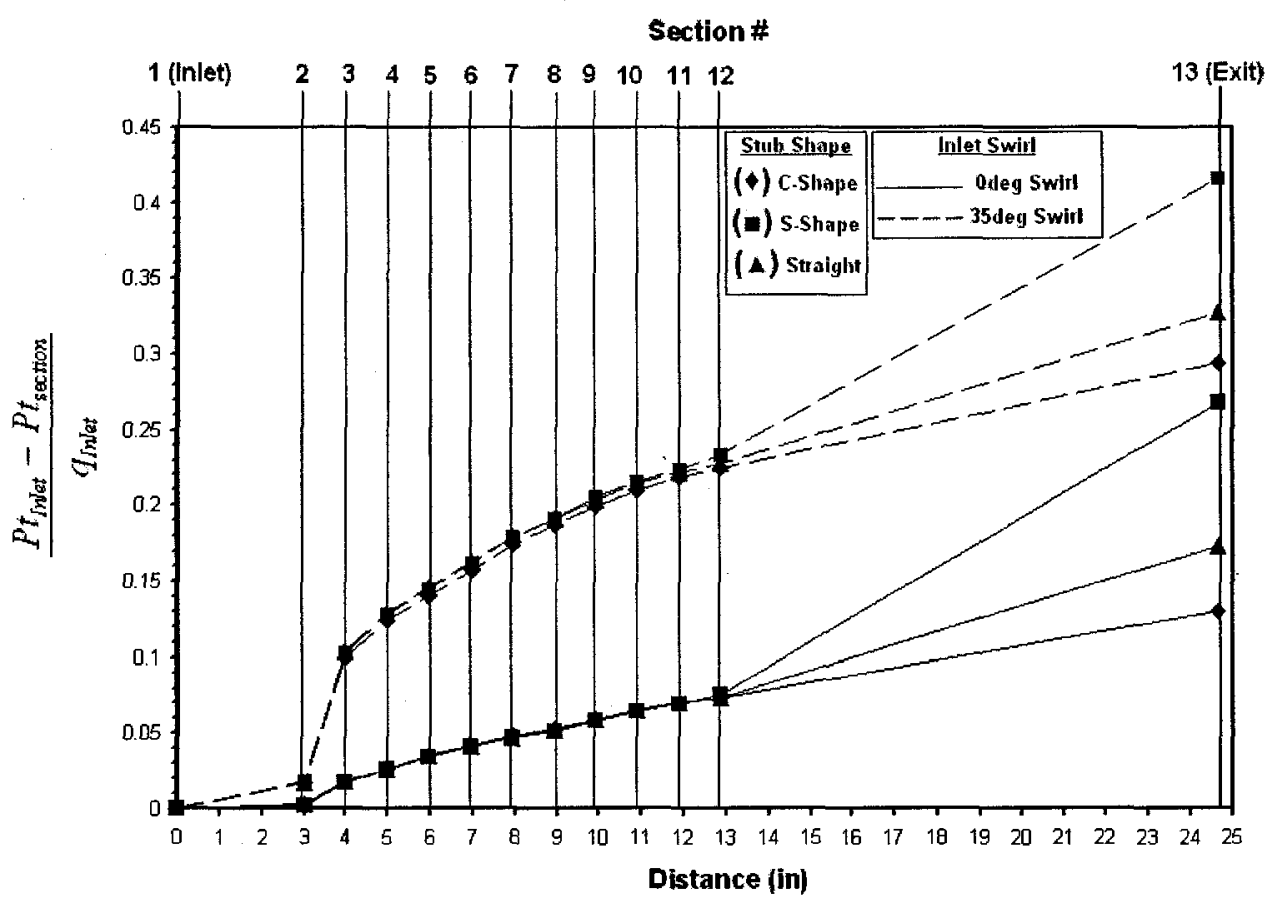

Figure 6-7: Total pressure losses calculated for C-shape, Straight, and S-shaped exhaust stubs (low inlet blockage)

The exhaust duct total pressure losses at the exhaust stub exit plane are dependent on the stub direction. However, the total pressure losses from exhaust duct inlet to the stub inlet are nearly independent of the stub direction. The graph in Fig. 6-7 demonstrates the general trend found in this study for a sample exhaust duct. For an inlet swirl angle of $0^{\circ}$ there is no discernable difference in the total pressure loss coefficient calculated from the inlet up to the stub inlet at section 12. After the stub inlet, there is a clear difference in the duct losses where the C-shaped stub demonstrates the lower losses with the next highest being a Straight stub followed by the S-shaped stub being the worst. The same trend is seen for higher inlet swirl conditions where variations in total pressure losses at a given section can be less then $5 \%$ up to the stub inlet.

\subsection{Effect of ECDA}

As demonstrated in Figs. 6-3 and 6-4, the flow structure in the annular to rectangular transition region can be quite complex. A two dimensional flow field becomes asymmetric 


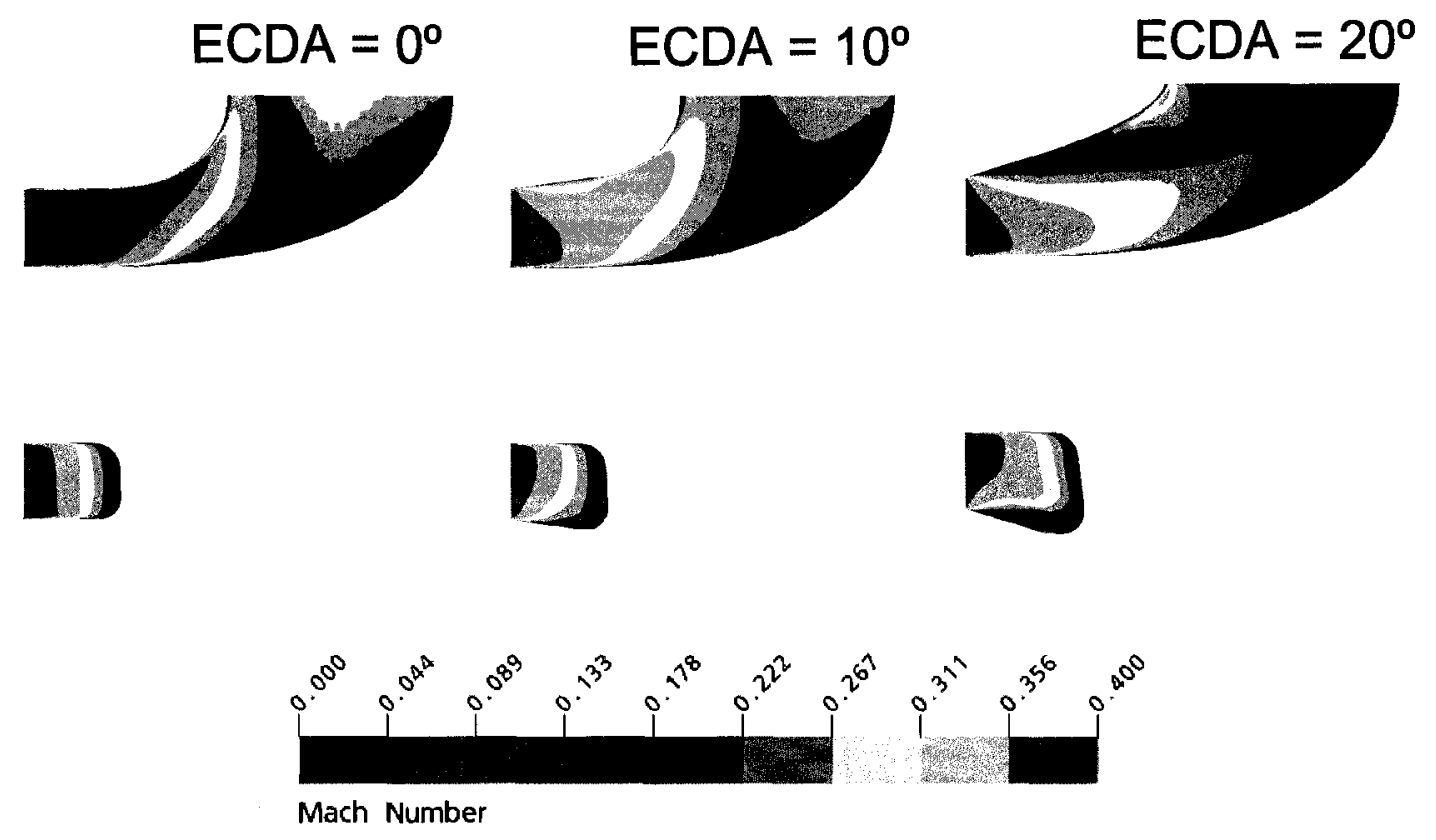

Figure 6-8: Mach number contours at mid-plane demonstrating the effect of ECDA (inlet conditions: nominal $0^{0}$ swirl with low blockage)

by the exit of the annular region at section 2 with flow separation occurring in the down stream sections. To produce lower losses downstream of the annulus, it is desirable to reduce the fluid Mach number as much as possible.

The effect of the ECDA tested on the flow field can be seen in Fig. 6-8, where Mach contours are plotted at mid plane of three exhaust ducts containing the different values of ECDA tested. It is apparent in the figure that an ECDA of $10^{\circ}$ led to the lowest mach numbers at the exhaust duct exit, with the highest occurring when no diffusion is present. The effect of too much diffusion in the annulus is seen in the figure, where flow separation is observed along the outer annulus walls of the duct with an ECDA of $20^{\circ}$.

The total pressure losses are calculated at the exit of the annulus of the ducts tested under inlet swirl conditions with low inlet blockage and presented in Fig. 6-9. The minimum losses are found to occur in all ducts with an ECDA of $10^{\circ}$. A trend of pressure losses increasing with increasing inlet swirl is also present in the figure with the exception of ducts $\mathrm{G}$ and $\mathrm{H}$ which both have ECDA of $20^{\circ}$. For these two ducts flow the flow separation is 


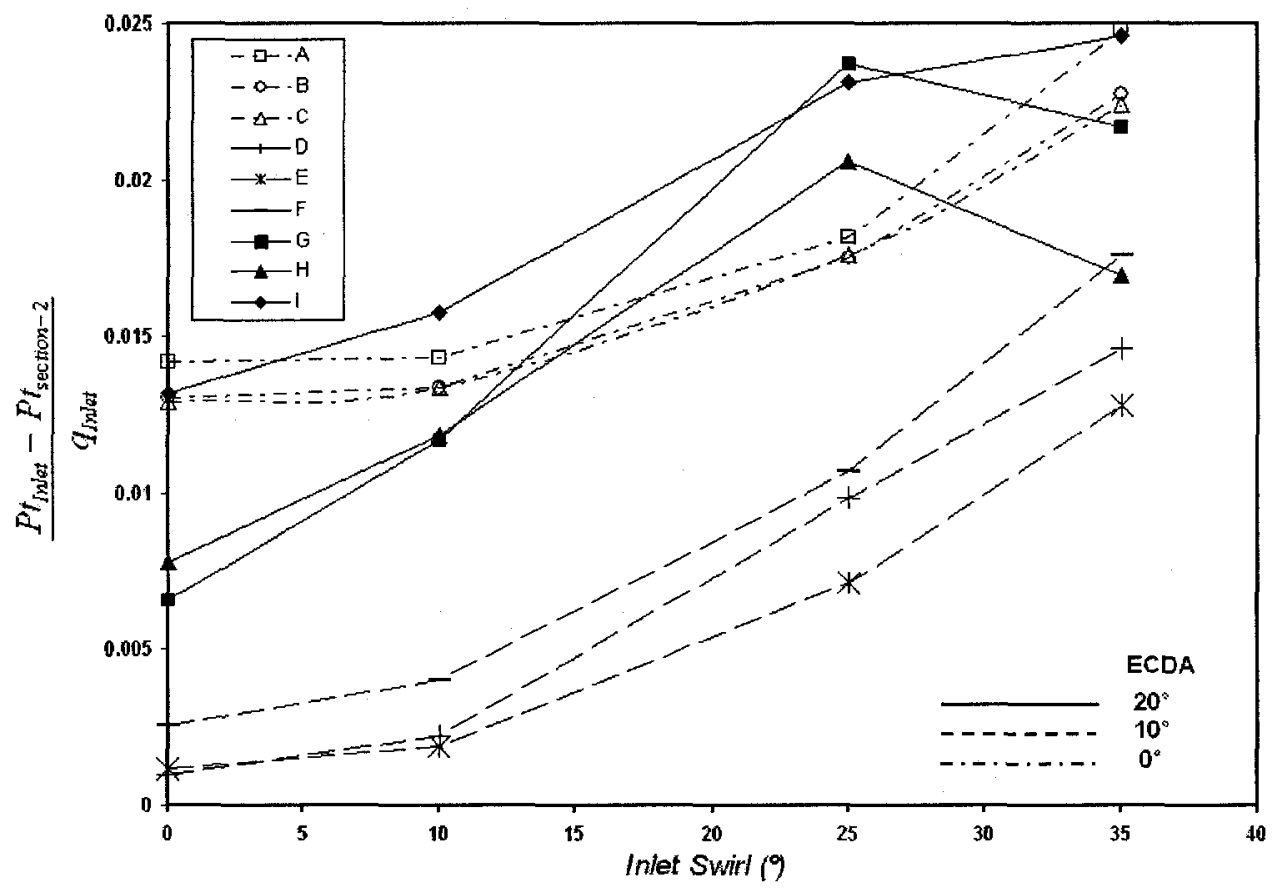

Figure 6-9: Total pressure losses as a function of ECDA calculated at exit of the annulus

seen to decrease from nominal $25^{\circ}$ inlet swirl to $35^{\circ}$ inlet swirl as shown by the contours of wall shear stress in Figs. 6-10 and 6-11.

\subsection{Effect of Wedge Angle}

As it was explained in Sec. 6.1 the largest increase in losses is always found to occur between section 2 and 3. It is between these two sections that we find the flow splitter. Figure 6-12 demonstrates the effect of increasing wedge angle on the pressure losses for three exhaust ducts with the same ECDA upstream of section 2. At nominal $0^{\circ}$ inlet swirl all three duct produce similar losses however as inlet swirl is increased the exhaust duct with an $80^{\circ}$ wedge angle produces lower losses at $25^{\circ}$ inlet swirl. To examine why the three exhaust duct are not similar at an inlet swirl of $25^{\circ}$, Mach contours are presented in Fig. 6-13 at a given cross-section cutting through the flow splitter. It is evident in the figure that the flow has separated along the suction surface of the exhaust ducts with wedge angles of $10^{\circ}$ and $45^{\circ}$ while the flow remains attached in the duct with the $80^{\circ}$ wedge angle. 

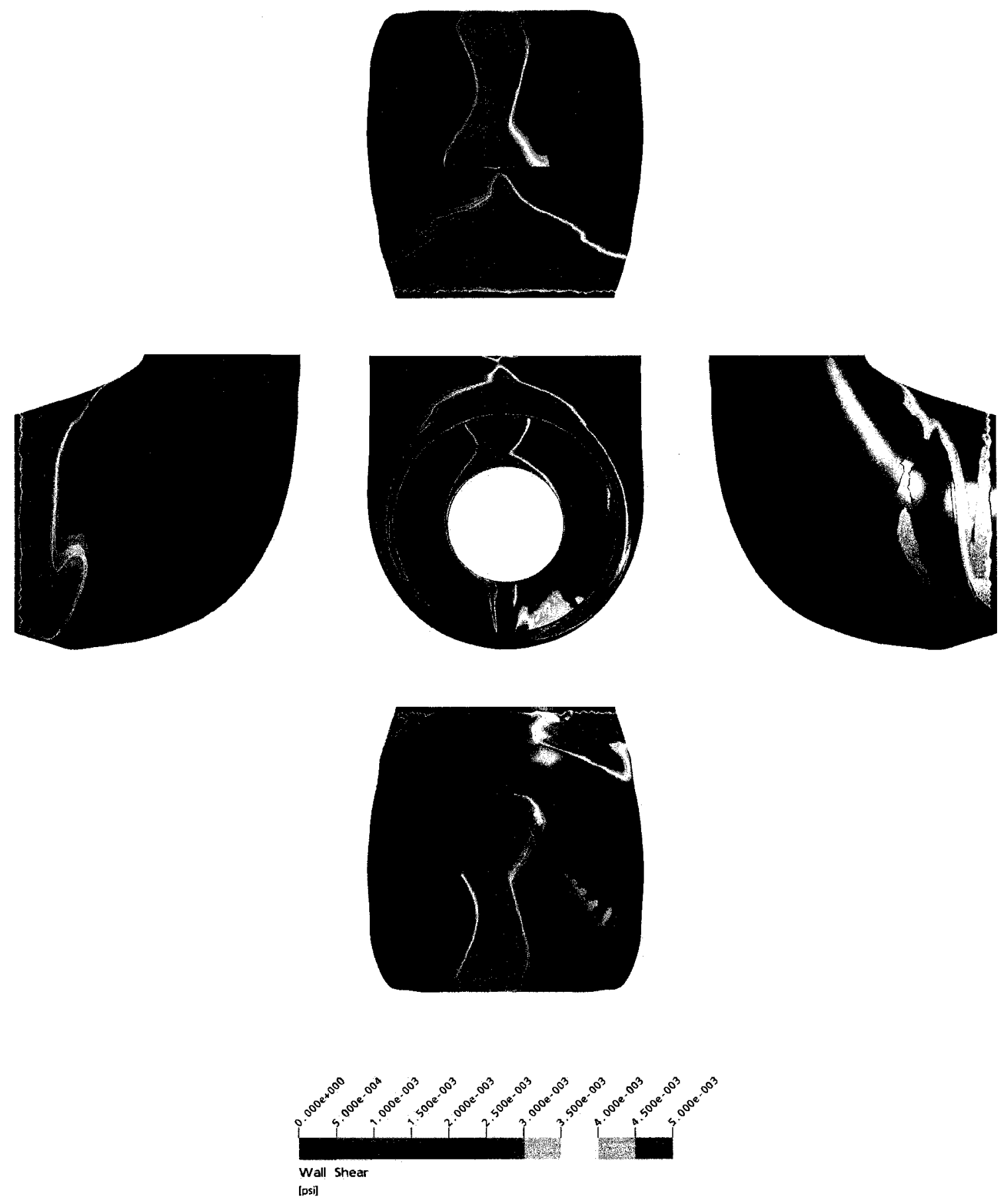

Figure 6-10: Wall shear stress contours (nominal $25^{0}$ swirl) 

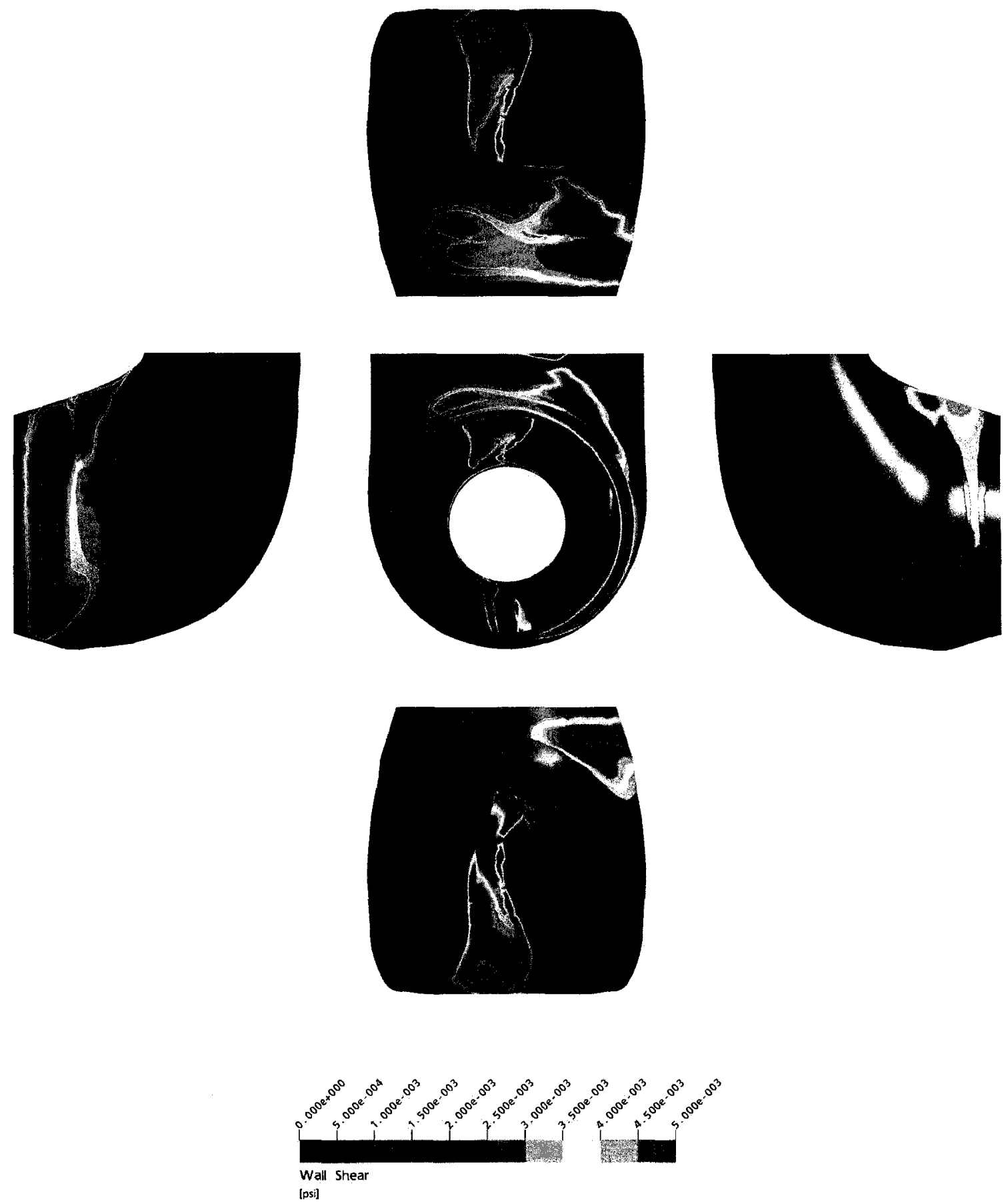

Figure 6-11: Wall shear stress contours (nominal $35^{0}$ swirl) 


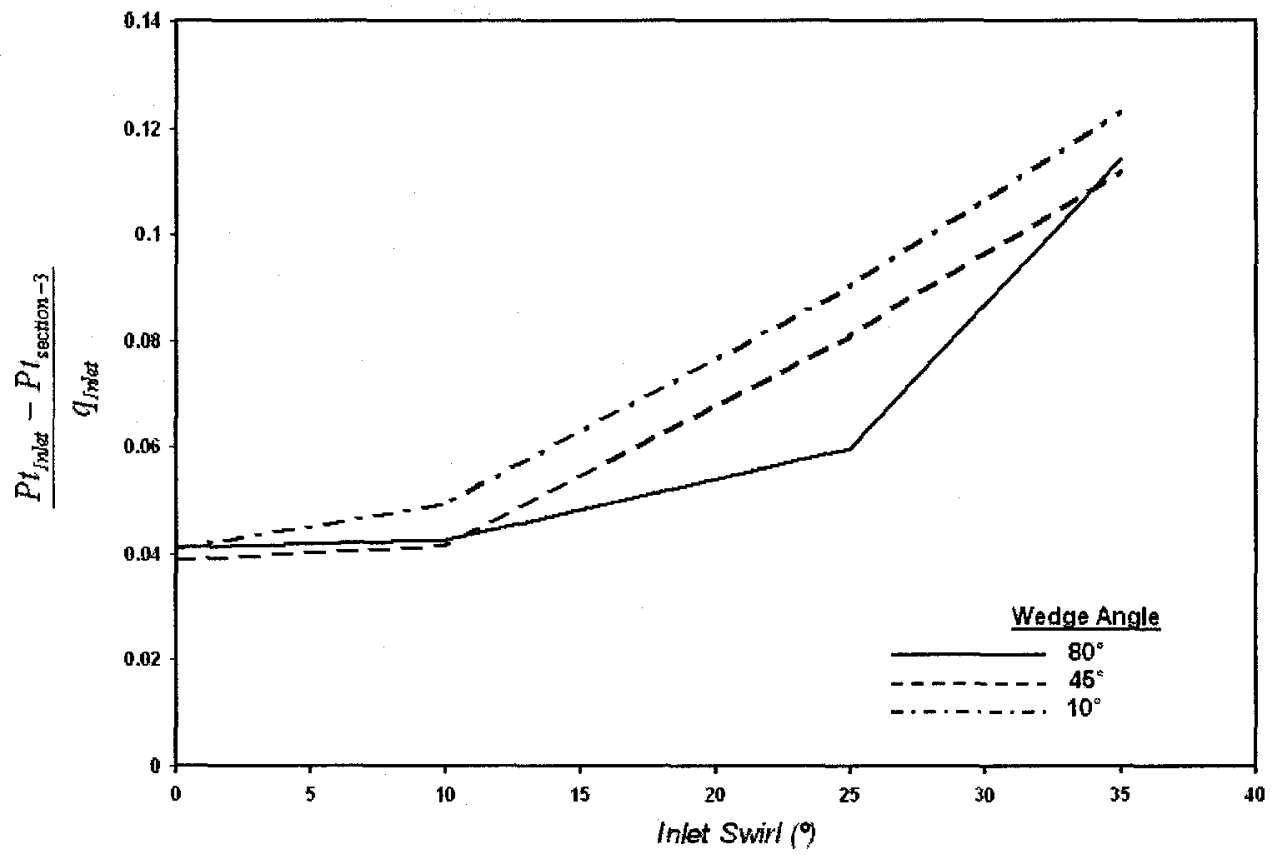

Figure 6-12: Total pressure losses as a function of wedge angle calculated at section 3

\subsection{Effect of Aspect Ratio and Area Ratio in the Annular to Rectangular Transition Region}

The effect of aspect ratio and area ratio in the annular to rectangular transition region on exhaust duct performance is difficult to quantify from the results of this analysis, because they are under the influence of the geometric parameters that precede them (such as ECDA and Wedge Angle). It can be seen from the test matrix in Table 3.5 that the influence of the aspect ratio and area ratio can not be demonstrated independently from the other geometric parameters. Some conclusions can be made when exhaust ducts are compared under no inlet swirl and low inlet blockage where those preceding geometric influences are at a minimum. Figures 6-14 and 6-15 present normalized static pressure and Mach number of four exhaust ducts which represent the combinations of aspect ratio and area ratio which are at the corners of the envelop tested. The normalized wall static pressure contours in Fig. 6-14 demonstrate that gradients on the wall are largest when the aspect ratio and area ratio are at the minimum. This is unfavorable since large pressure gradient will tend to promote flow separation off the duct surface. The Mach number contour plots at mid plane 

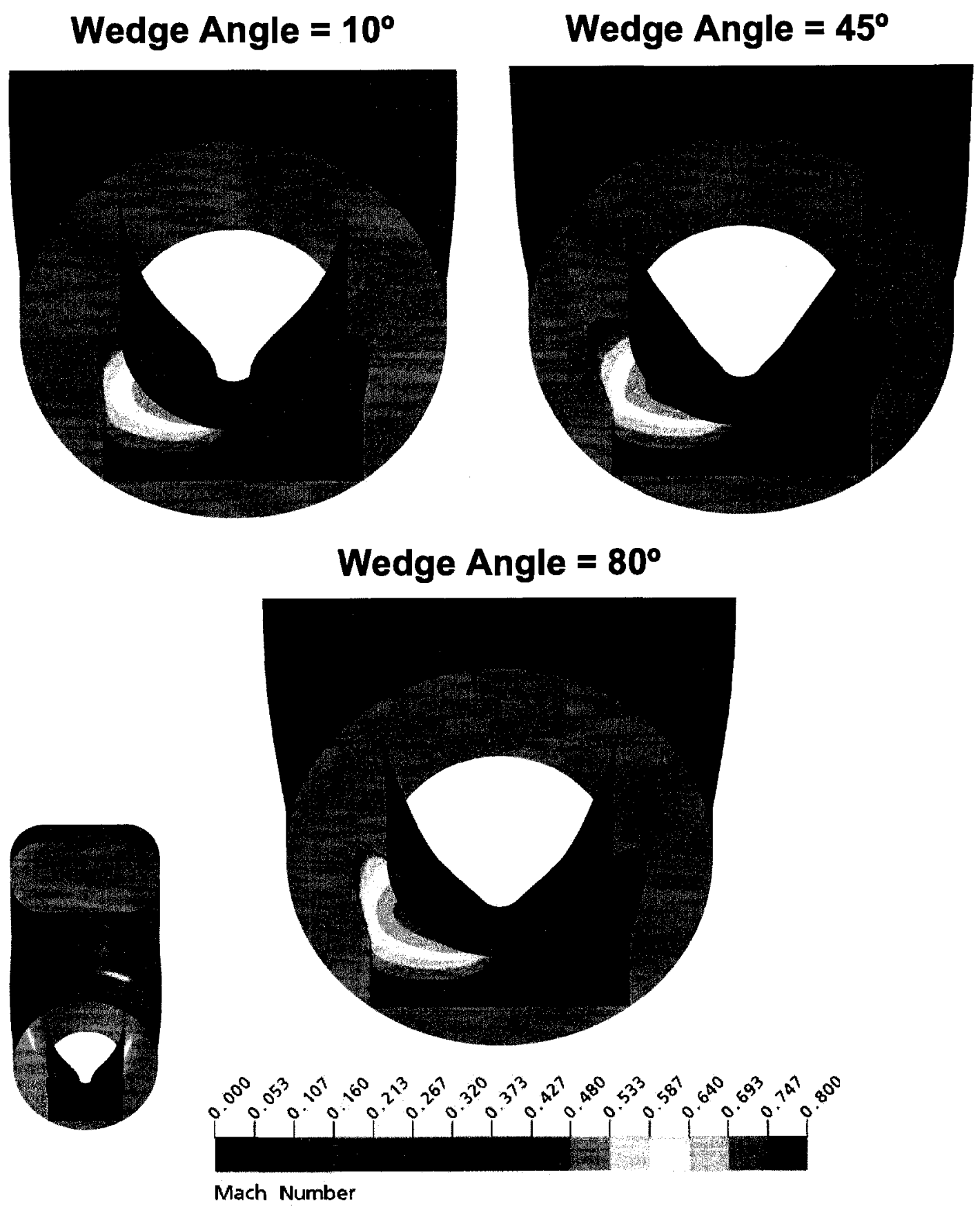

Figure 6-13: Mach number contour plot on a plane through the flow splitter 

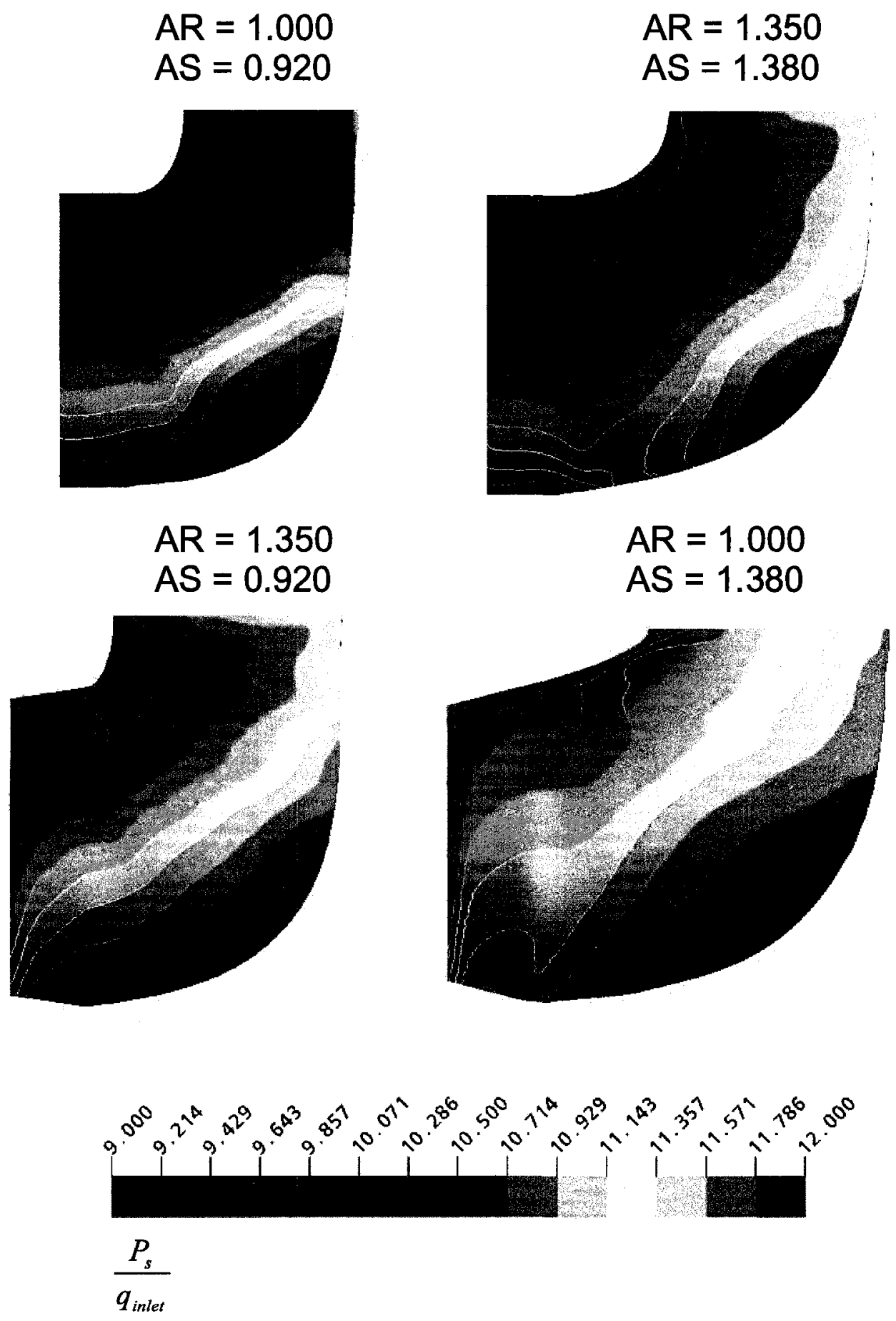

Figure 6-14: Normalized wall static pressure contours demonstrating the effect of aspect ratio and area ratio in the annular to rectangular transition region 

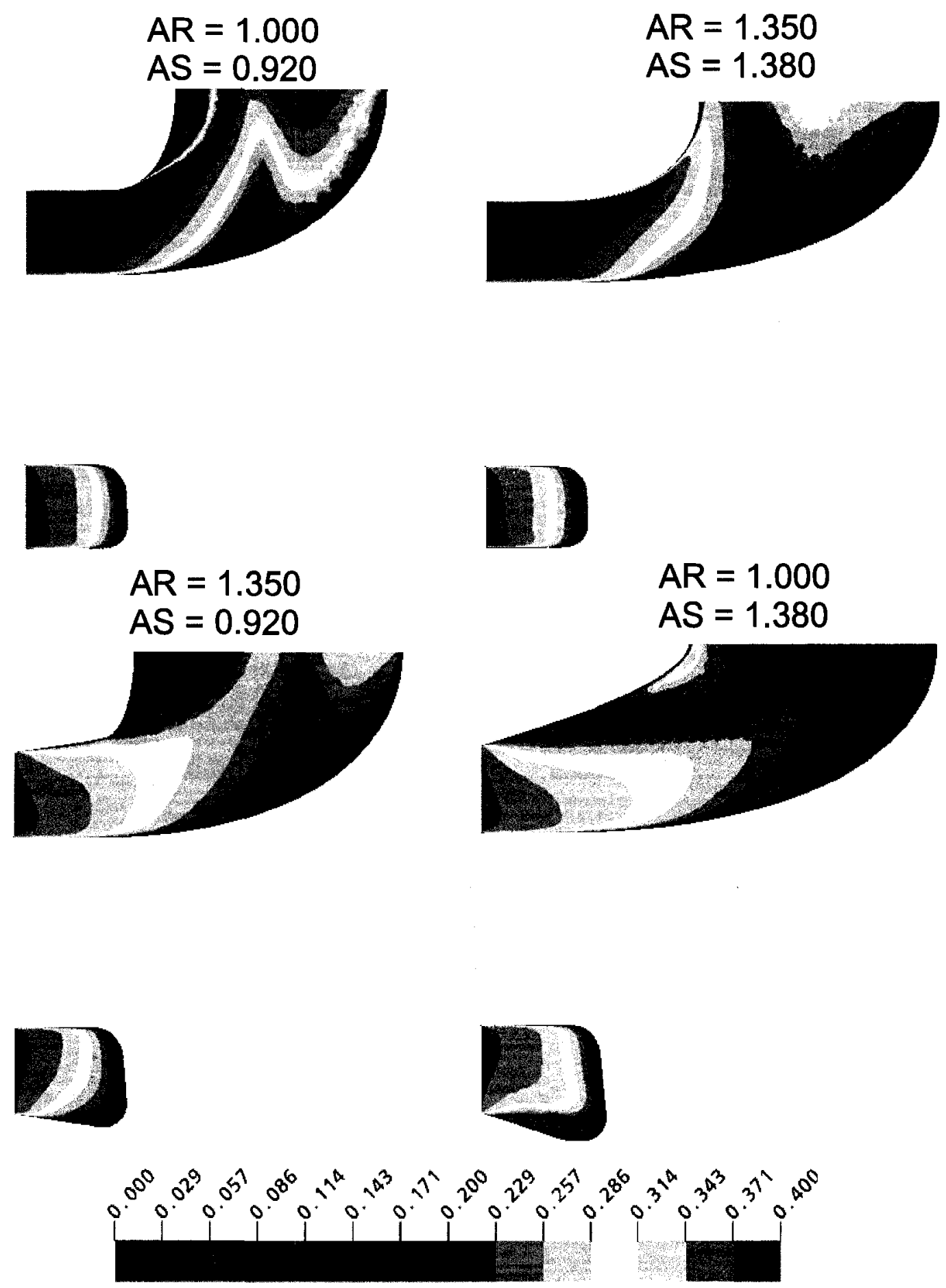

Mach Number

Figure 6-15: Mach number contours demonstrating the effect of aspect ratio and area ratio in the annular to rectangular transition region 
in Fig. 6-15 support this theory demonstrating that small aspect ratios do lead to flow separation off the inner surface of the exhaust duct reducing the available flow area in the streamwise direction. It can be concluded that it is favorable to have the largest available aspect ratio to maintain an attached flow.

\subsection{Effect of Inlet Boundary Layer Blockage}

It has been found that the total pressure losses increase as a result of increasing inlet boundary layer blockage. As seen in Fig. 6-16 a thick inlet boundary layer is more prone to flow separation with the point of separation progressing closer to the inlet for thicker inlet boundary layers. These results where expected when flowing against a positive pressure gradient. The exhaust ducts with $20^{\circ}$ ECDA demonstrated to have the most severe flow separation over the other ECDA tested. The global effect of inlet blockage on all exhaust ducts tested can be fully seen the Figs. 6-17 and 6-18. With low inlet blockage, there no distinct relation to other tested geometric parameters, however increased blockage levels have demonstrated higher levels of losses related to large inlet diffusion angles. It is apparent in both figures that for medium and large inlet blockage, the exhaust ducts tested with $20^{\circ}$ ECDA have an overall higher trend of total pressure losses. When comparing medium to high inlet blockage the same loss trends are found only at higher levels. 


\section{Inlet blockage}

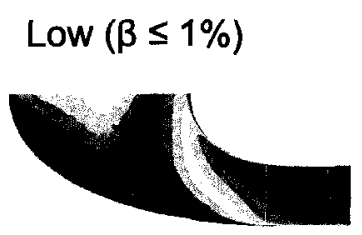

Medium ( $4 \% \leq \beta \leq 8 \%)$

High $(\beta \geq 10 \%)$
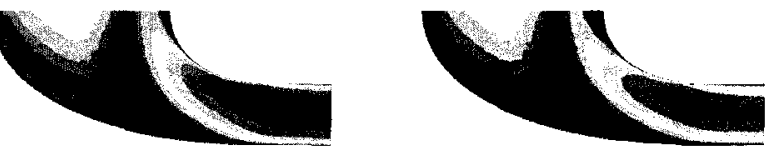

$E C D A=0^{\circ}$
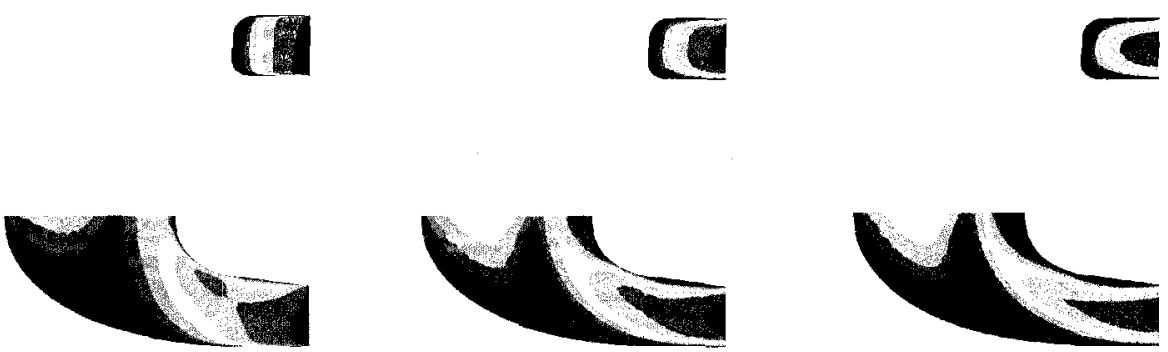

$E C D A=10^{\circ}$
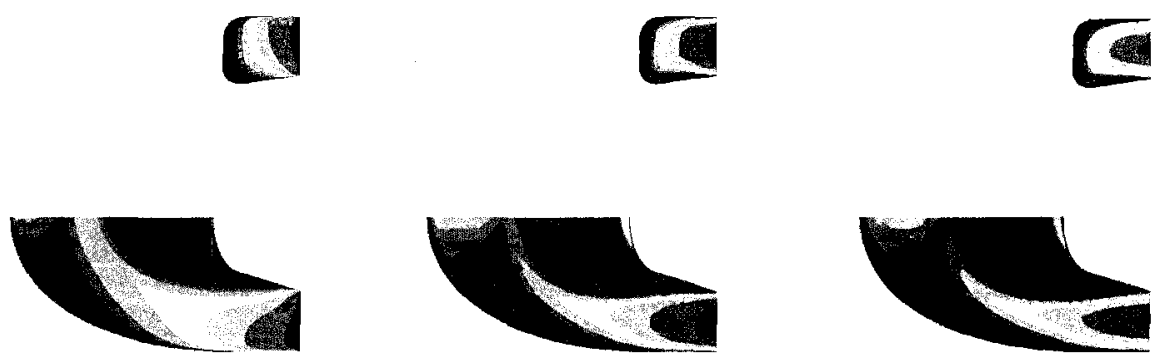

$E C D A=20^{\circ}$
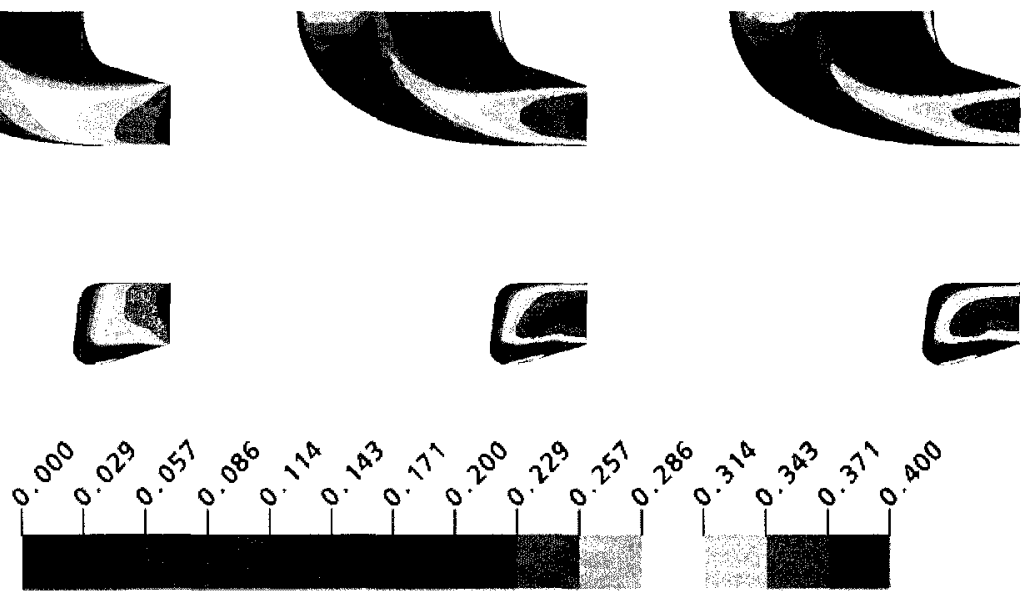

Mach Number

Figure 6-16: Mach number contours demonstrating the effect of inlet blockage on three exhaust ducts with ECDA of $0^{\circ}, 10^{\circ}$, and $20^{\circ}$ 


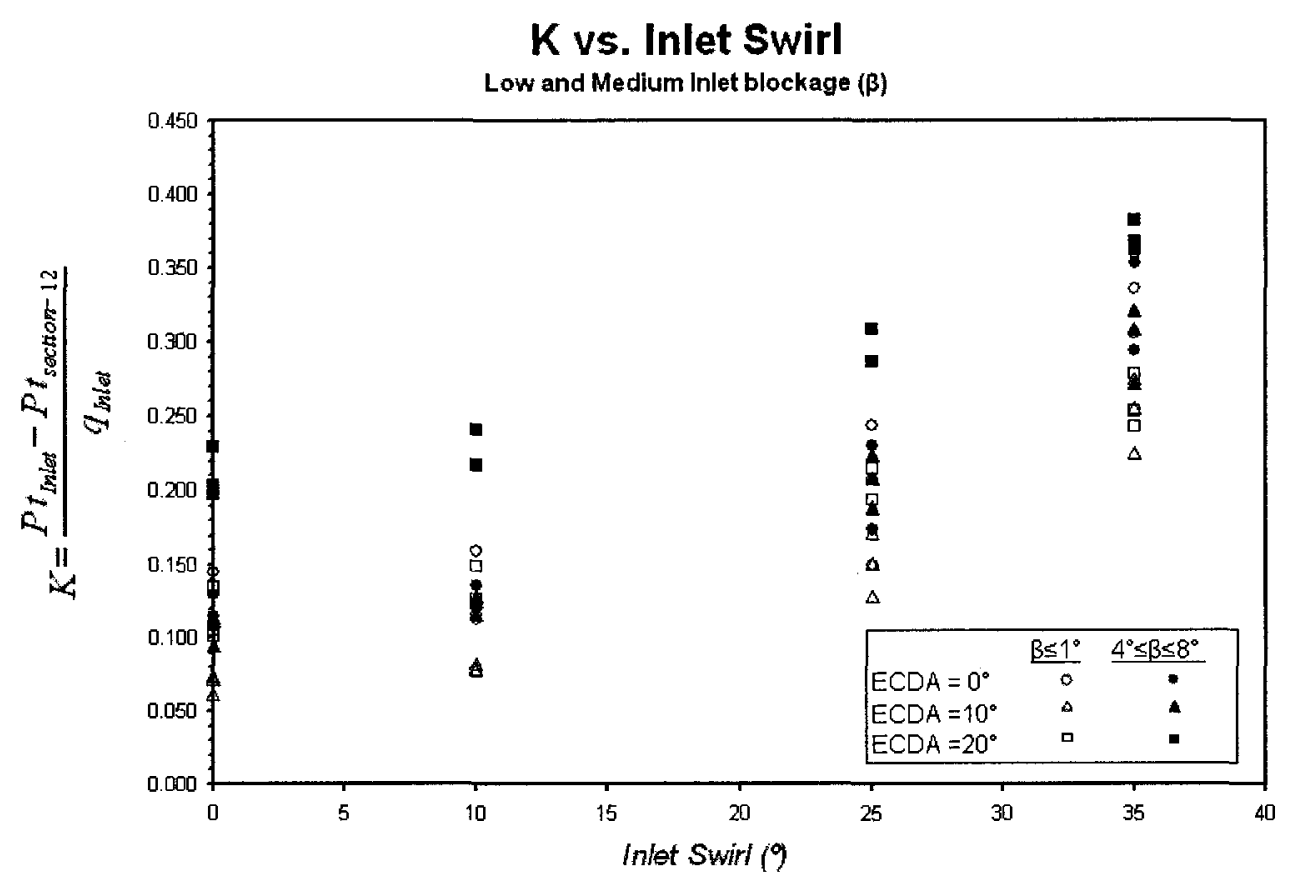

Figure 6-17: Total pressure loss coefficient vs. inlet swirl for low and medium inlet blockage

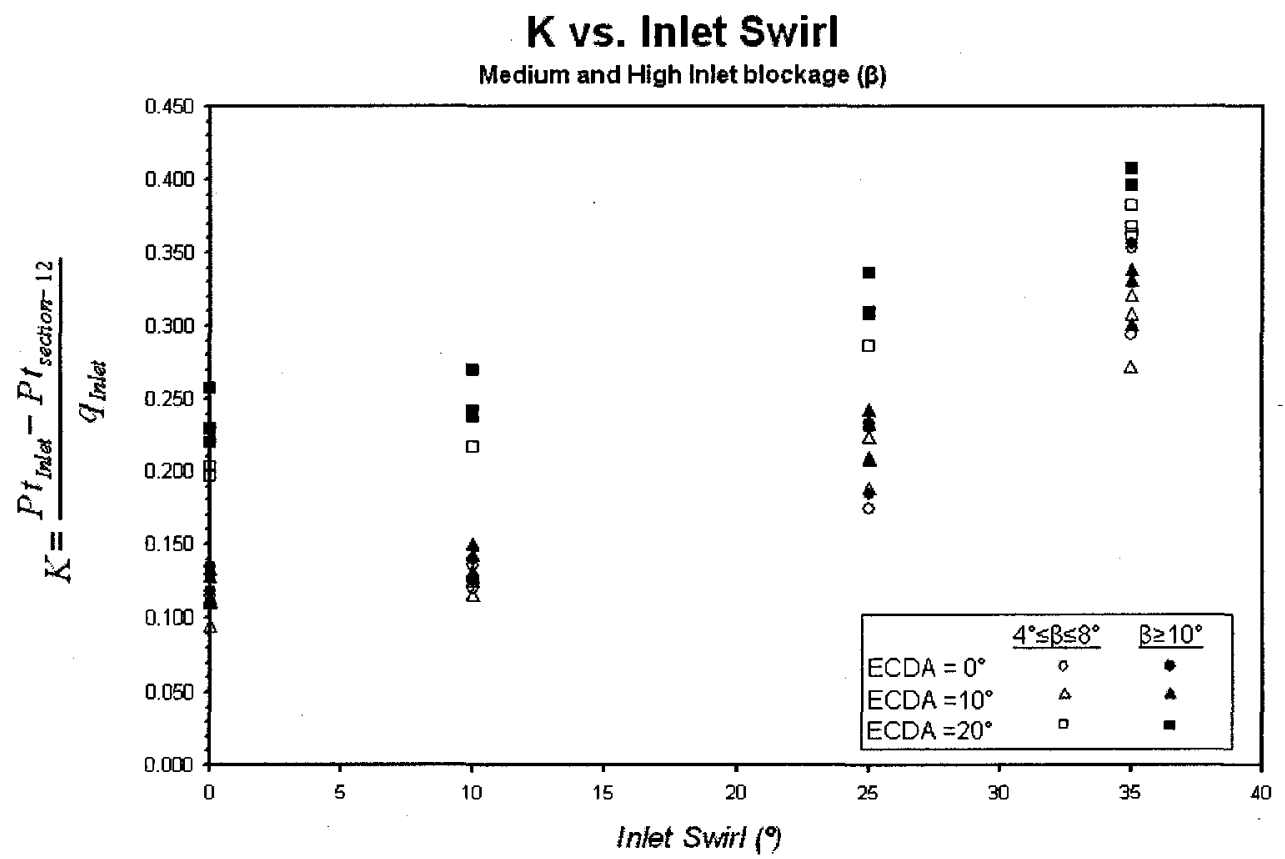

Figure 6-18: Total pressure loss coefficient vs. inlet swirl for medium and high inlet blockage 


\section{Chapter 7}

\section{Correlation of the Total to Total}

\section{Pressure Loss}

A correlation is presented in the following sections where the total to total pressure loss coefficient is related to the geometric and aerodynamic parameters based on the data produced in the computational flow simulations. The resulting correlation serves as a suitable tool for designers when involved in the preliminary design of annular to rectangular exhaust ducts.

The results of the parametric study demonstrate that the geometry of the exhaust duct produces a highly asymmetric flow field, under the influence of swirl, and flow separation is almost unavoidable when the later is combined with inlet blockage. The favorable approach to produce the loss correlation would be to build upon an exact solution of the Navier Stokes equation which can be easily be interpreted by a designer. However, an exact solution to the Navier Stokes equations does not exist for this complex geometry therefore a numerical approach must be taken.

The development of a loss model can follow one of the following two approaches; 1 ) a purely mathematical approach where a reduced order model or a surrogate model is constructed from available data (eg. Artificial Neural Networks [25]), and 2) a physical approach where a numerical correlation is composed of different terms each of which represent the effect of one flow feature contributing to the loss. The first approach is a black 
I VS. AR

only those points whose B1 is loss than or equal to 0.06

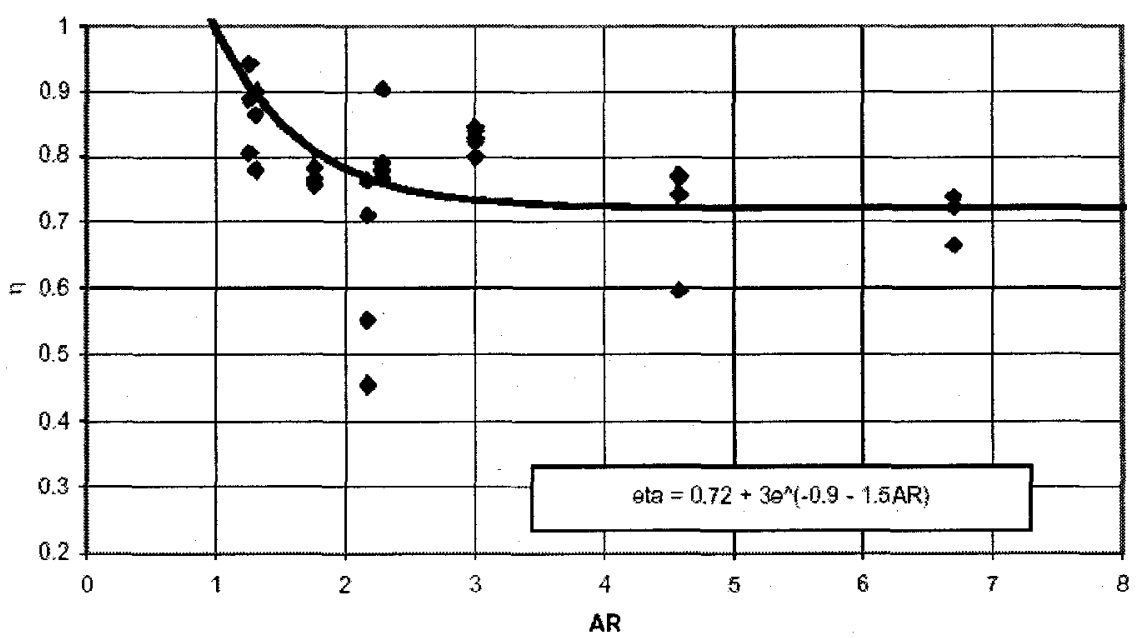

Figure 7-1: Diffuser effectiveness versus area ratio with low level inlet aerodynamic blockage and no inlet swirl [7]

box to the designer and does not provide the user with the same feedback as do physical correlations produced by curve fitting. Through curve fitting, the correlation will provide the user with a visual understanding of the functional relations which can even take on a physical meaning of the data being analyzed. The approach taken in this work is the second one, where the data is correlated through a curve fitting technique similar to what was used by Japikse [7] where he correlated annular diffuser effectiveness using available published data.

\subsection{Japikse Correlation of Annular Diffusers}

To start the process Japikse has first identified that area ratio is the dominant variable related to diffuser effectiveness, once the data was screened for blockage, and noting that inlet swirl has been to some extent taken care of through the definition of $C p_{i}$ in equation 2.3. He then plotted the data versus area ratio, Fig. 7-1, and discovered that the data followed an exponential trend.

With the equation found in Fig. 7-1, Japikse was able to move on to the other variables 


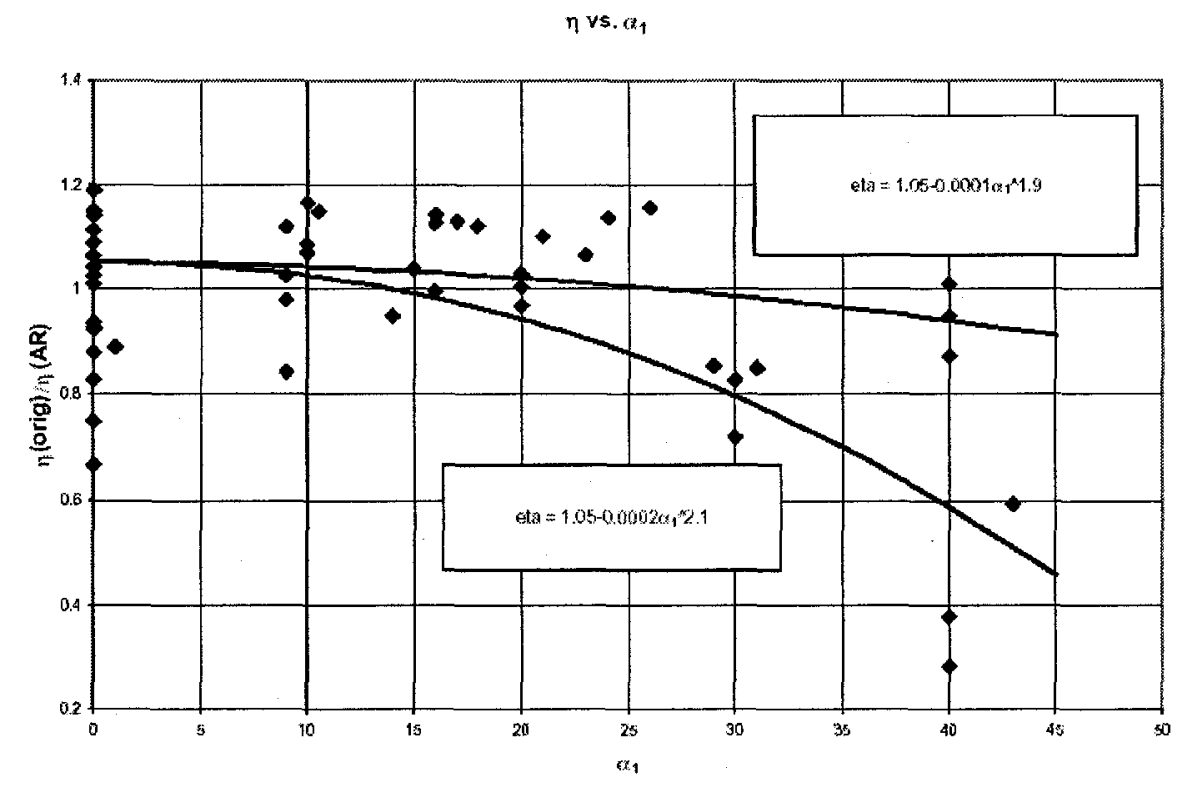

Figure 7-2: Diffuser effectiveness with principle geometric effects removed including data at all levels of inlet aerodynamic blockage [7]

by removing the effect of area ratio by dividing out the data by the new expression as presented in Fig. 7-2. In this figure, the data has been plotted versus inlet swirl and has revealed two trends. The upper trend represents diffusers experiencing mild stall while the lower trend represents diffusers with substantial stall.

The data was again divided by the new equations defined in Fig. 7-2, and the with the effects of area ratio and inlet swirl removed from the data Japikse then moved on to inlet blockage as shown in Fig. 7-3. From this figure Japikse has determined that there are two trends which have been defined. The lower trend (common blockage, "A"), which is seen to passes through the square symbols, is data from Coladipietro et al. [18] where tests were conducted at two different blockage levels. In these testes, inlet conditions consisted of a clean uniform velocity profile where only the boundary layer thickness was varied. The upper trend (classical profile blockage, "B") which shows that diffuser performance improves with increasing inlet blockage comes from inlet conditions where the boundary layer becomes fully developed and contains increase levels of turbulence and vorticity. Dividing the data again by the new equations, Japikse represented the data versus inlet blockage in Fig. 7-3 


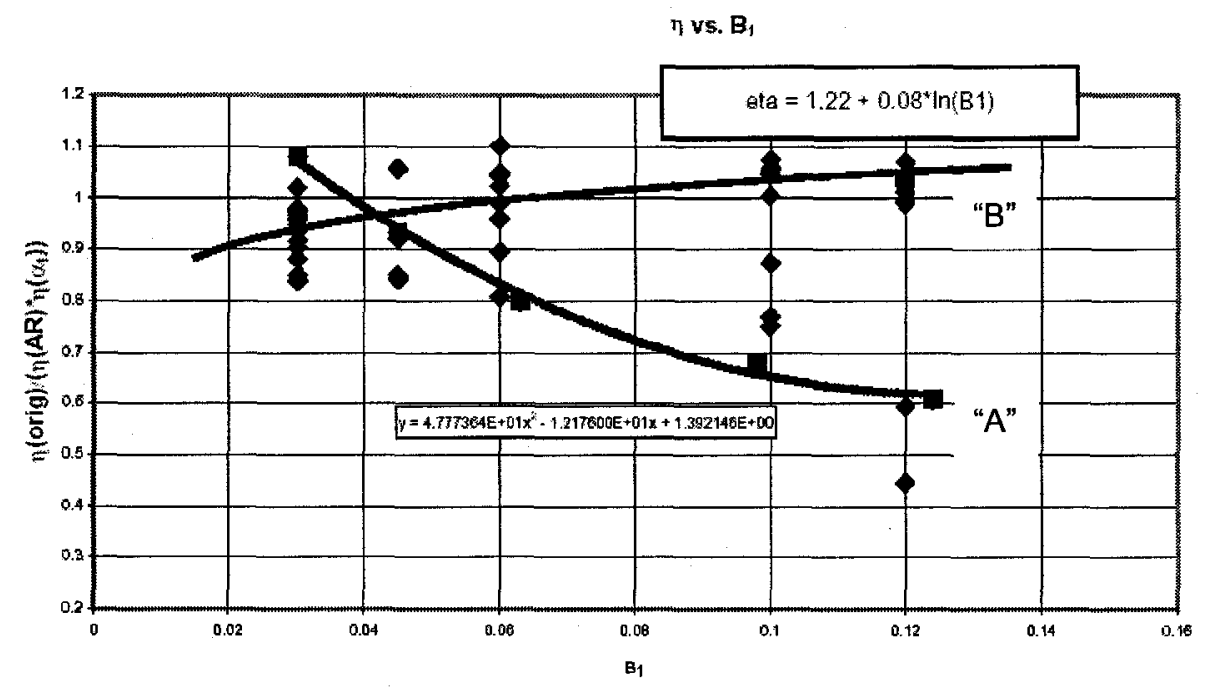

Figure 7-3: Diffuser effectiveness with the principle effects of geometry and inlet swirl removed according to preceding correlations [7]

to reveal that the data has collapsed to a value of $1 \pm .10$.

The resulting set of equations produced by studying the data trends is the following:

$$
\begin{gathered}
C p=C p_{i}\left(\alpha_{1}, r_{2} / r_{1}, b_{2} / b_{1}\right) \eta(A R) \eta\left(\alpha_{1}\right) \eta\left(B_{1}\right) \\
\eta(A R)=0.72+3 e^{(-0.9-1.5 A R)} \\
\eta\left(\alpha_{1}\right)=1.1-0.0001 \alpha_{1}^{1.9} \ldots \ldots \ldots \ldots . . \text { delayed stall } \\
\eta\left(\alpha_{1}\right)=1.1-0.0002 \alpha_{1}^{2.1} \ldots \ldots \ldots \ldots \ldots . . . \text { early stall }
\end{gathered}
$$

$\eta\left(B_{1}\right)=47.77364 B_{1}^{2}-12.17600 B_{1}+1.392146 \ldots \ldots$ curve $A$, common blockage

$\eta\left(B_{1}\right)=1.22+0.08 \times \ln \left(B_{1}\right) \ldots \ldots \ldots \ldots \ldots \ldots \ldots \ldots$ curve $B$, classical profile blockage

Now that the diffuser effectiveness can be predicted, Japikse suggest that a reasonable first order estimate of the diffuser total pressure loss coefficient can be calculated using Eq. 2.6 ( $\mathrm{Cp}$ is evaluated from Eq. 7.1 and $C p_{i}$ from Eq. 2.3). 
ๆ vs. $B_{1}$

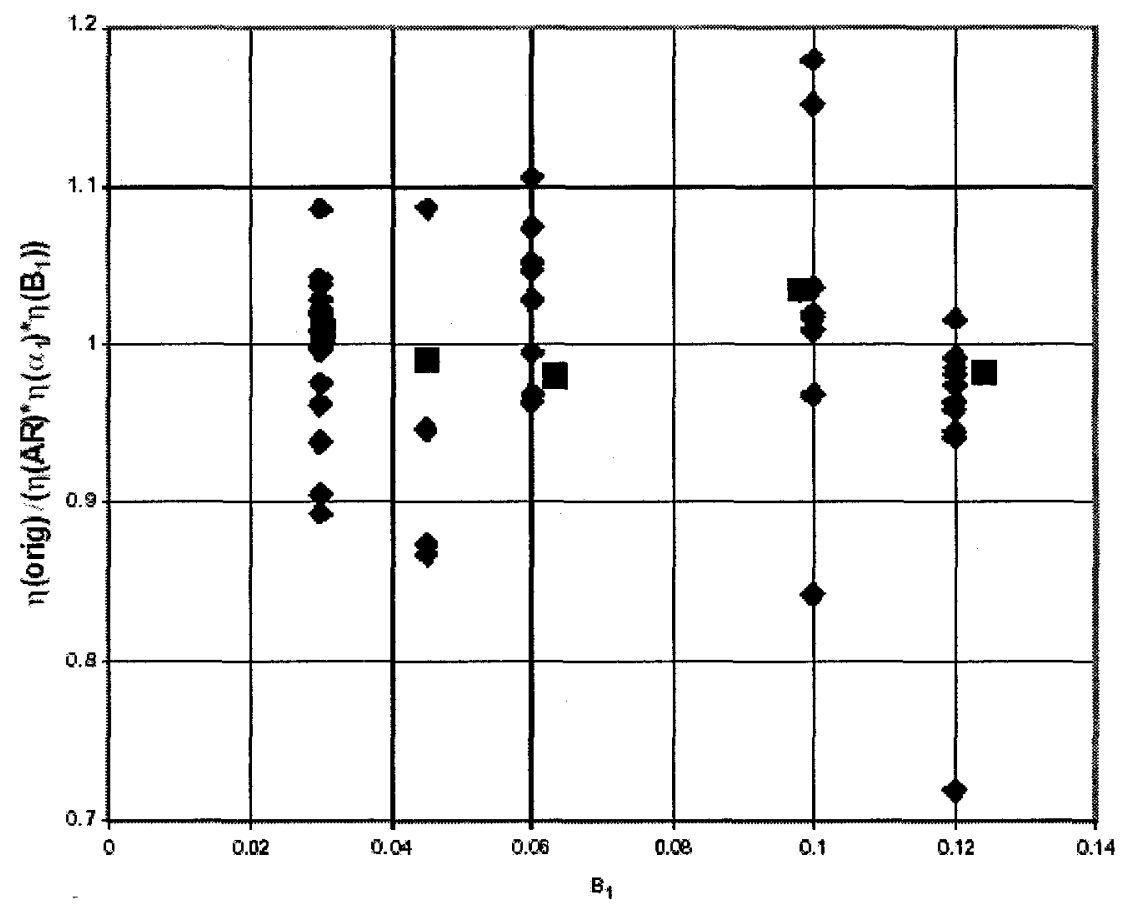

Figure 7-4: Diffuser effectiveness with the principle effects of geometry, inlet swirl, and inlet blockage removed [7] 


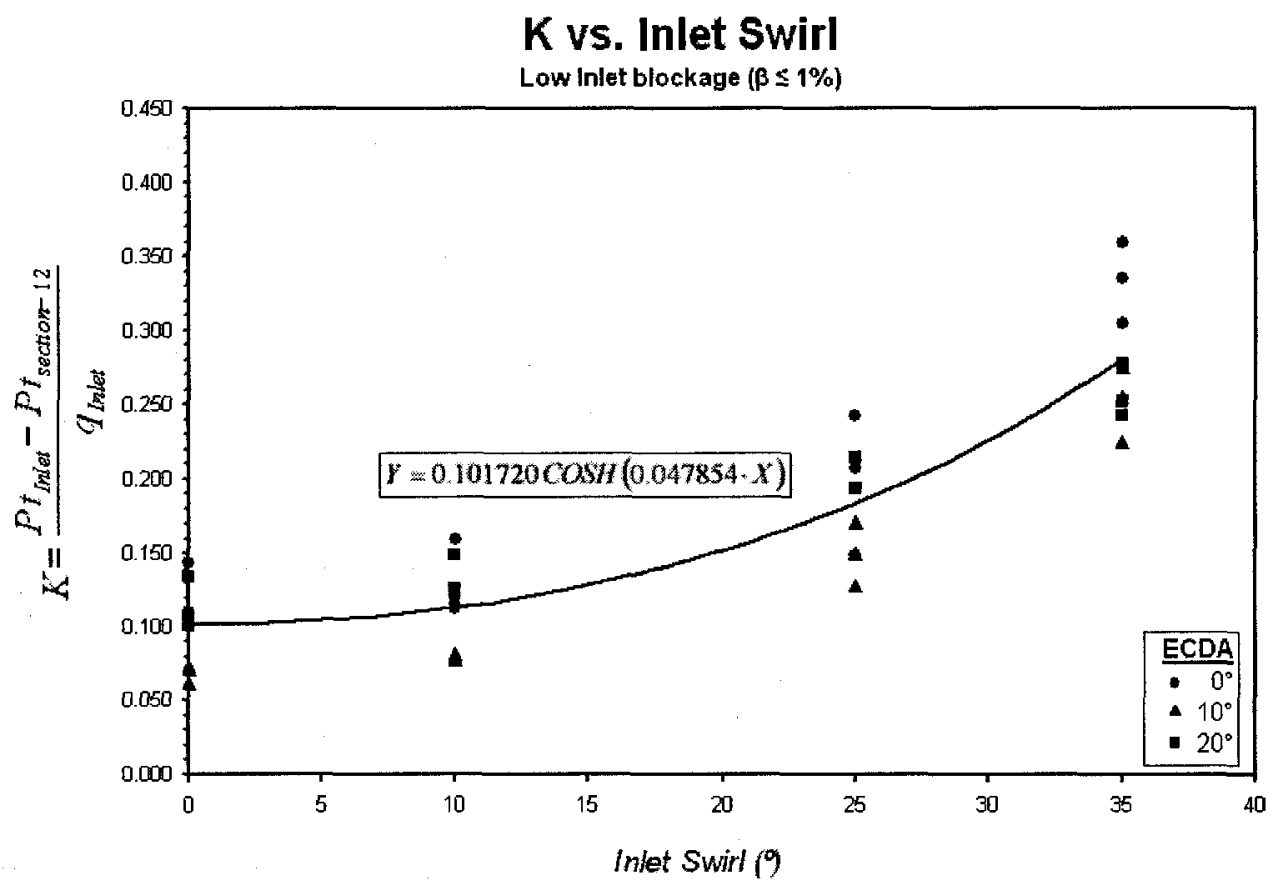

Figure 7-5: Total pressure loss trend in the exhaust for low inlet blockage

\subsection{Correlation of the CFD Results}

It was demonstrated in Sec. 6.2 that the total pressure losses in the exhaust duct are independent of the stub direction. For this reason it was chosen to correlate the exhaust duct losses independently of the stub losses. The following section presents a corelation to predict the total pressure losses between the inlet and section-12 as shown in Fig. 6-1. The correlations produced in this work have been found with the help of Windows Excel and the statistical package LAB Fit [26].

The fist parameter that demonstrated to have the first order impact on losses in the exhaust duct is inlet swirl. When inlet blockage was varied some distinct trends appeared which demonstrated different behaviors in the losses depending on the magnitude of diffusion taking place in the annular inlet of the exhaust duct. For low inlet blockage $(\beta \leqslant 1 \%)$ it is possible to find a single trend which can be used to normalize the data, Fig. 7-5. For medium inlet blockage $(4 \% \leqslant \beta \leqslant 8 \%)$ one trend has been defined for an ECDA of $20^{\circ}$ and one for the lower values tested, Fig. $7-6$. When high inlet blockage $(\beta \geqslant 10 \%)$ is present a 


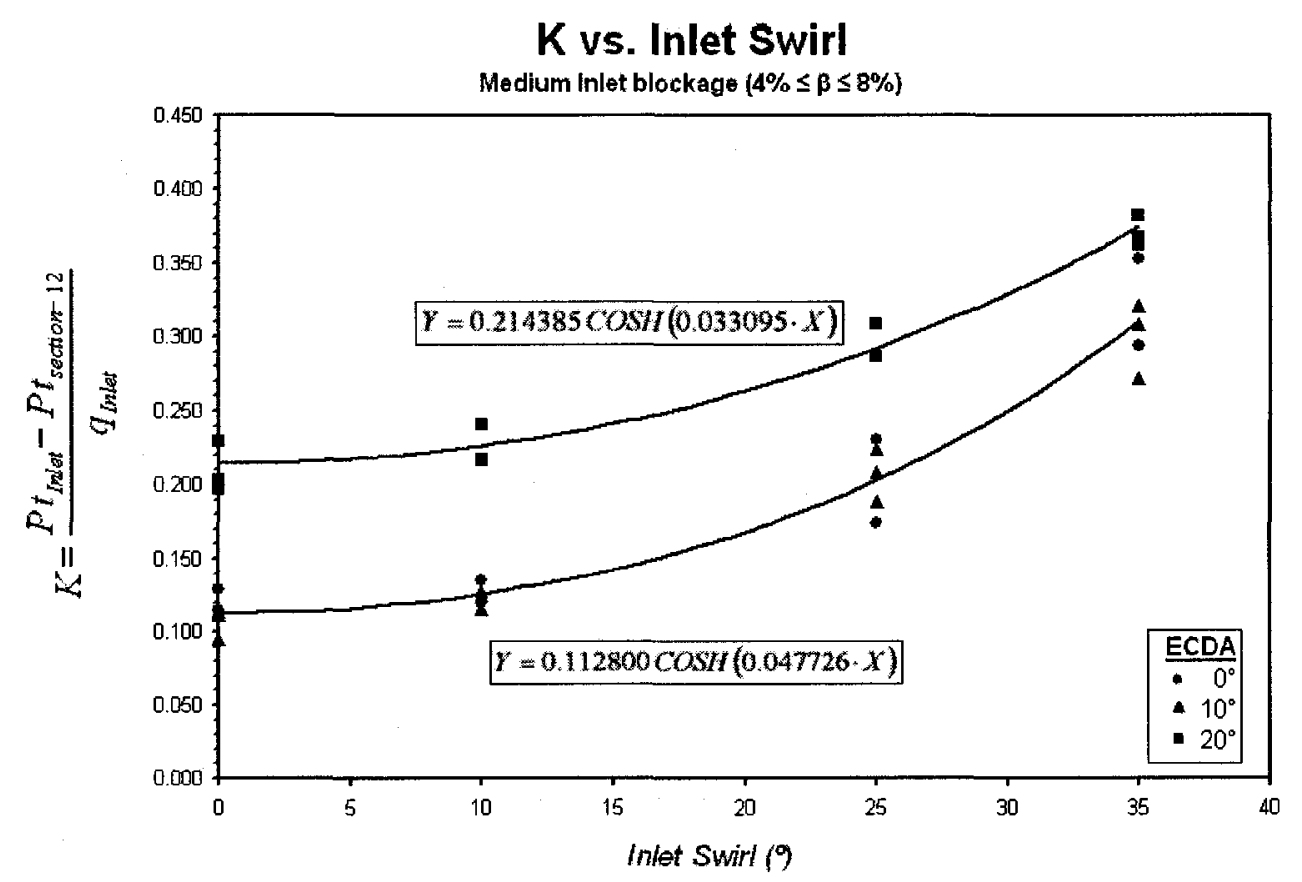

Figure 7-6: Total pressure loss trend in the exhaust for medium inlet blockage

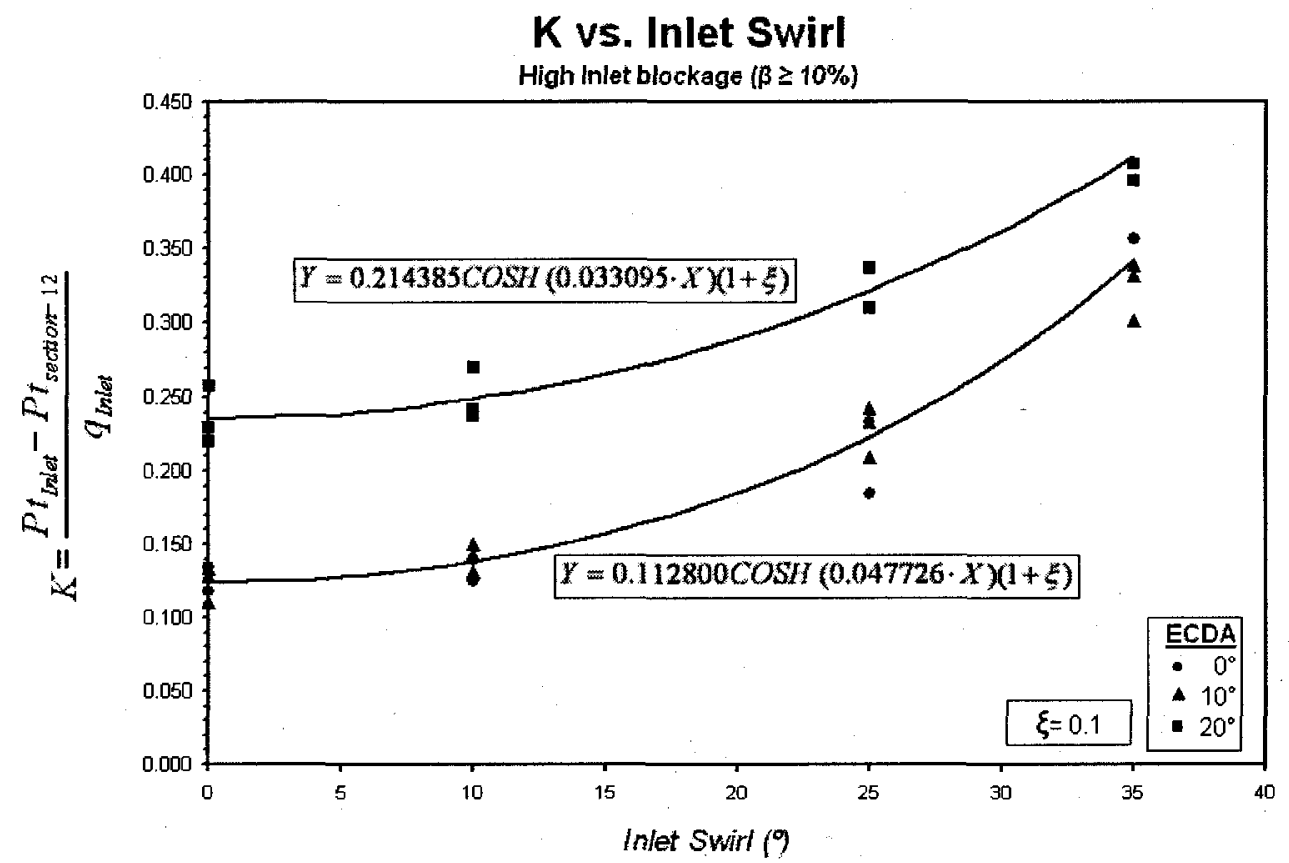

Figure 7-7: Total pressure loss trend in the exhaust for large inlet blockage 


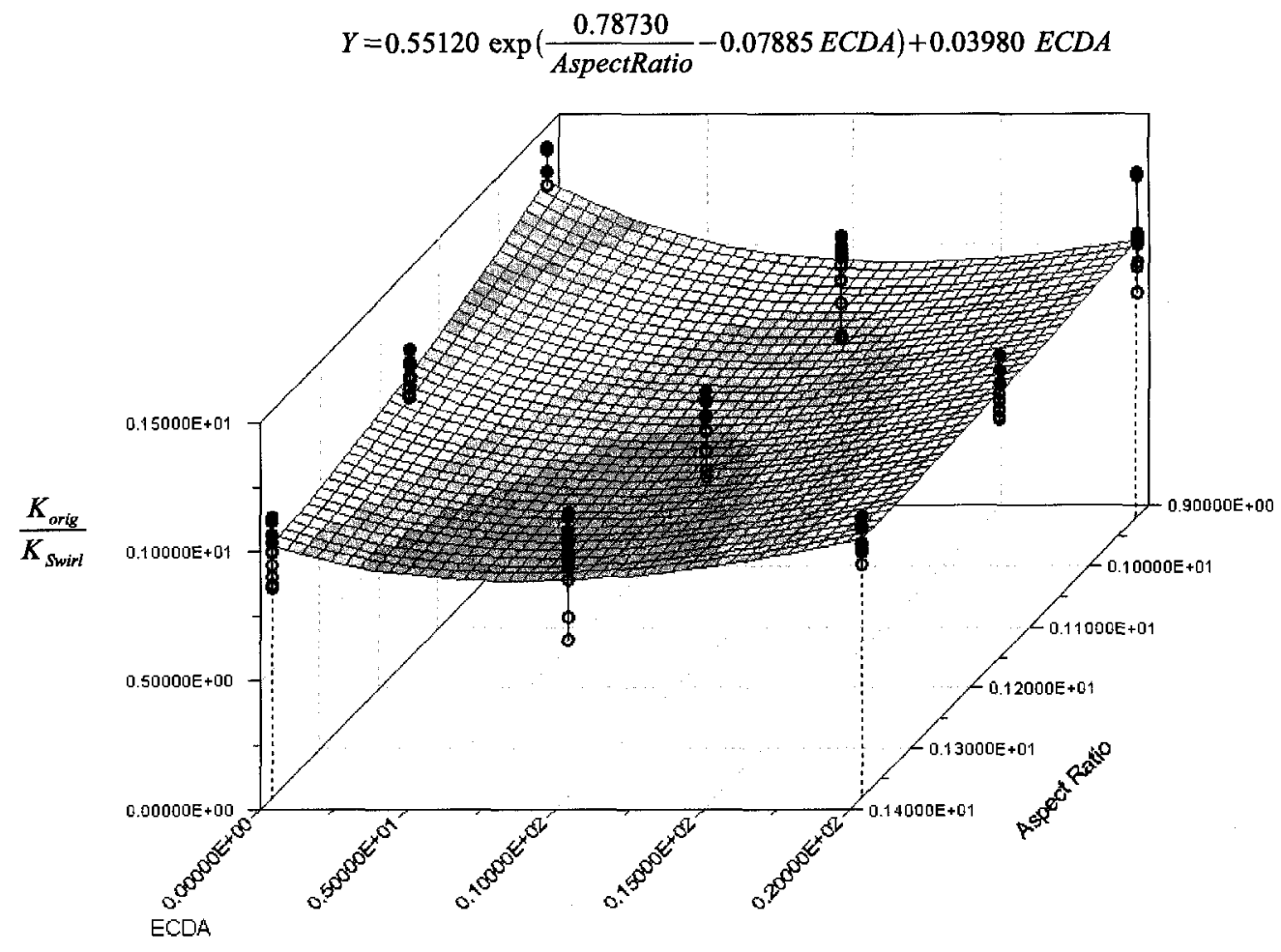

Figure 7-8: Surface passing through normalized losses for ECDA and aspect ratio (Solid circles are points lying above the surface and hollow circles are points lying below)

correction factor is used to increase the level of the trends used to describe medium inlet blockage, Fig. 7-7. To calculate the loss due to intermediate ECDA and blockage levels, it is recommended to interpolate between the trends in Fig. 7-6 and then interpolated with Fig. 7-5 for smaller inlet blockage or select a smaller correction factor for higher inlet blockages.

The equations derived above have considered the contribution to the losses due to the effect of swirl with some consideration to the annular inlet section defined by ECDA. After normalizing the data with those equations the effect of swirl with some consideration to ECDA have effectively been removed and the other contributing parameters can now be evaluated.

The next parameter considered is the aspect ratio of the exhaust duct. A good fit to the data could not be found after trials at correlating the aspect ratio to the normalized 


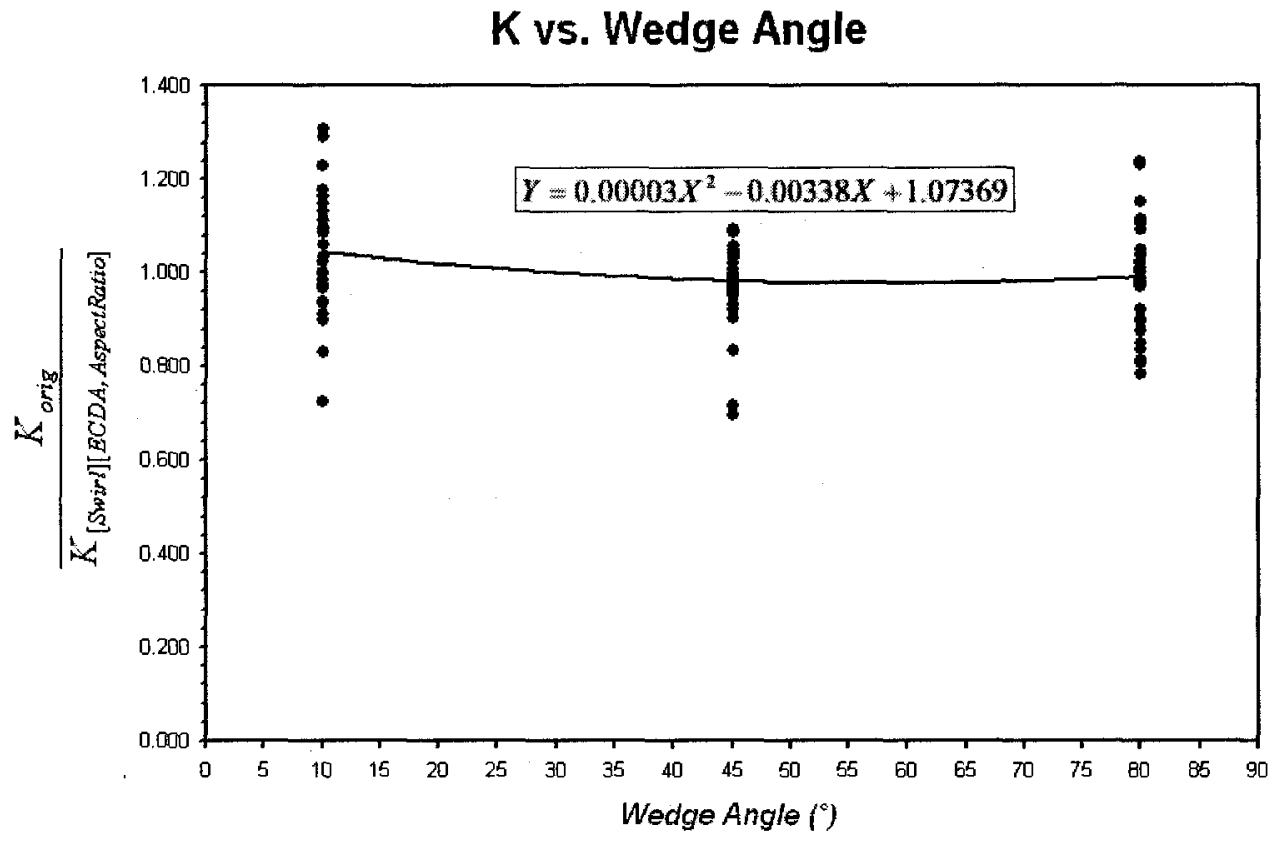

Figure 7-9: Normalized losses versus wedge angle with the effects of blockage, swirl, ECDA, and aspect ratio removed

data demonstrating that some other parameter is influencing the losses. It was discovered that there may still remain some influence of the annular inlet effecting the data and when correlating ECDA with the aspect ratio a surface could be passed through the data with satisfactory results, Fig. 7-8. The surface plot in Fig. 7-8 supports the results presented in Sec. 6.3 and 6.5 demonstrating that it is favorable to have a large aspect ratio and that there is an optimum ECDA between $0^{\circ}$ and $20^{\circ}$.

The data are again normalized with respect to the surface equation defined above and plotted against the values of wedge angles tested, Fig. 7-9. A trend is visible and a polynomial fit demonstrates that there is an optimum wedge angle near $60^{\circ}$. Once the data are normalized again for this trend we see in Fig. 7-10 that there is only minimum influence of the area ratio on the normalized pressure losses and a polynomial fit is made to the data.

The final result of the correlation can be seen in Fig. 7-11. It is demonstrated that $70 \%$ of the data falls within $\pm 10 \%$ error and $85 \%$ of the data falls within $\pm 15 \%$ error. A more detailed breakdown of the accuracy of the correlation is shown in Table 7.1. the resulting 


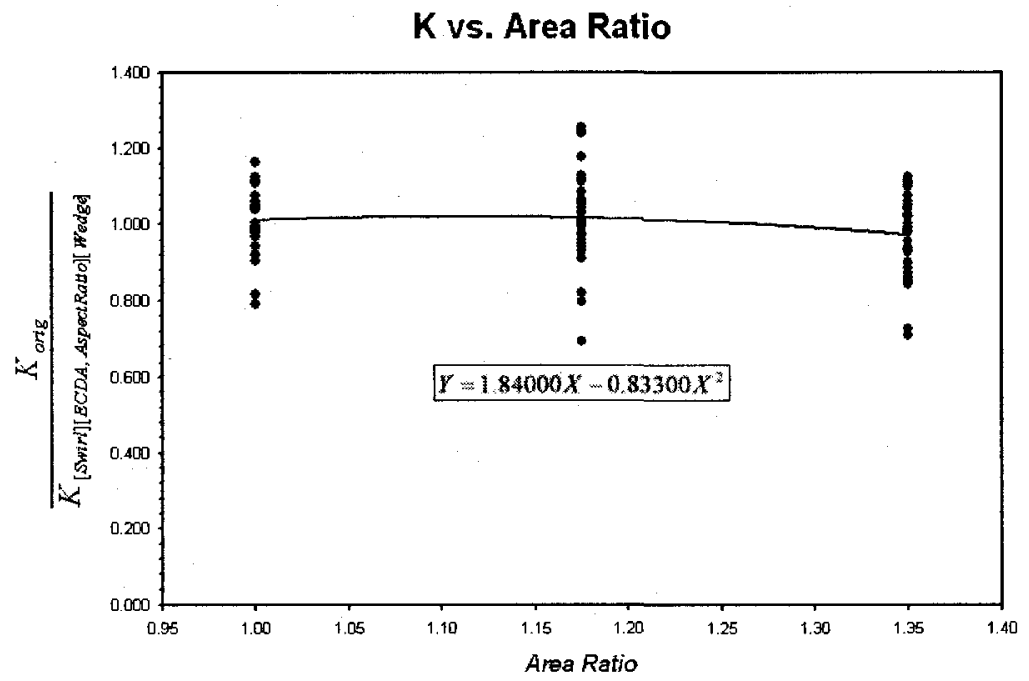

Figure 7-10: Normalized losses versus area ratio with the effects of blockage, swirl, ECDA, aspect ratio, and wedge angle removed

Table 7.1: Break Down of Correlation Accuracy

\begin{tabular}{cc} 
Error $\left(\mid\left(K_{C F D}-K_{\text {Correlation }} \mid\right)\right.$ & Data Points $(\%)$ \\
\hline $0.00-0.02$ & 72 \\
$0.02-0.04$ & 22 \\
$0.04-0.06$ & 6
\end{tabular}




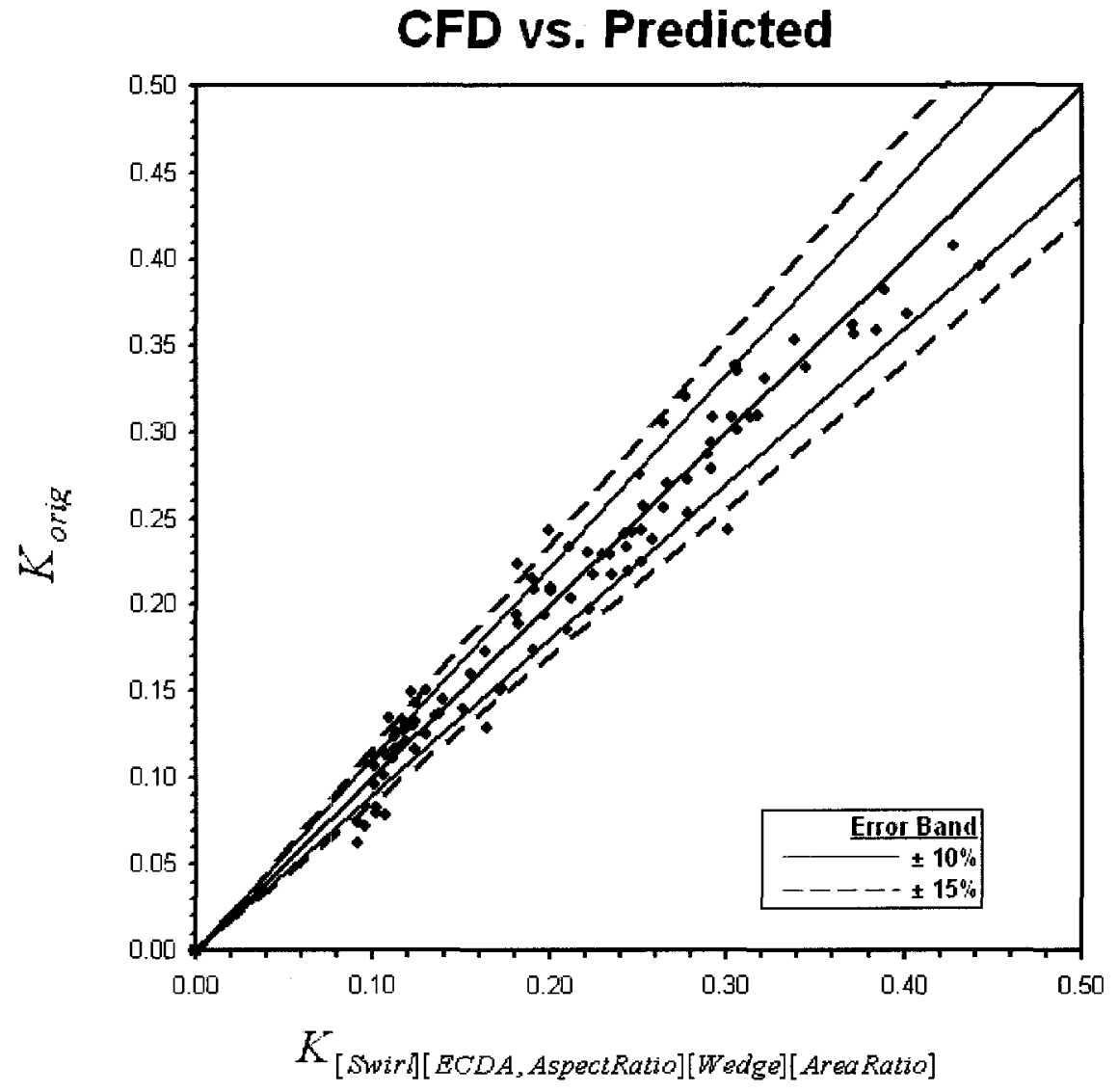

Figure 7-11: Plot of CFD losses vs. predicted losses between inlet and section-12 
set of equations are as follows:

$$
\begin{gathered}
K=K(\text { Swirl }, X \text { blockage }) K(E C D A, \text { AspectRatio }) K(\text { WedgeAngle }) K(\text { AreaRatio }) \\
K(\text { Swirl }, \text { low blockage })=0.101720 \cosh (0.047854 \alpha) \ldots \ldots \ldots \ldots \ldots \ldots \text {.ll ECDA }
\end{gathered}
$$




\section{Chapter 8}

\section{Conclusion and Recommendation}

\subsection{Conclusion}

A parametric CFD study has been carried out where key geometric and aerodynamic parameters are varied and the effect on the total pressure loss is observed. The results of the CFD data were then used to produce a correlation to predict the duct losses. Based on this work the following conclusions are made:

1. Inlet Swirl

- Inlet swirl was demonstrated to have a high order effect on the pressure loss in the exhaust duct. The minimum pressure losses occur when the swirl is at a minimum. The introduction of inlet swirl causes an asymmetric flow field yielding to flow separation with increased pressure losses.

2. Stub direction

- pressure loss at the exhaust duct exit was found to be independent of the stub direction. The highest losses occur in the S-shaped stubs as a results unfavorable flow separation due to pressure gradients.

3. ECDA

- The degree of ECDA demonstrated that too little and too large of an ECDA is not favorable. The optimum value of ECDA resulting in the minimum velocity at 
the inlet to the annular to rectangular transition region without flow separation is between $7^{\circ}$ and $10^{\circ}$.

4. Wedge Angle

- The three wedge angles tested perform similarly for inlet swirls up to $25^{\circ}$. At this swirl angle flow separation on the suction surface of the flow splitter occurs on the wedge angles of $10^{\circ}$ and $45^{\circ}$ and is delayed for the $80^{\circ}$ flow splitter. At $35^{\circ}$ inlet swirl the $80^{\circ}$ wedge flow splitter would separate on the suction surface. It is desirable to have a large wedge angle for reduced pressure loss.

5. Aspect Ratio and Area Ratio

- It was difficult to quantify the effect of these parameters since they proceed the other geometric parameters. A small aspect ratio and area ratio lead to pressure gradients that can result in flow separation.

6. Inlet Boundary Layer Blockage

- The total pressure losses increase with increasing inlet blockage. Exhaust ducts with ECDA of $20^{\circ}$ performed to worst of the geometries tested for medium and high inlet blockage.

7. Correlation of the CFD Results

- The Taguchi Design provided a test matrix that provided the minimum amount of exhaust duct models to successfully capture the resulting effects on the total pressure loss coefficient. The CFD data has been correlated to the total pressure loss coefficient with reasonable accuracy.

\subsection{Recommendation}

Examining the results of the current study, the following recommendations are made for the loss modeling of non-symmetric gas turbine exhaust ducts using CFD: 
1. As future work, it is desirable to include the exhaust stubs in the correlation. To do this, first, a survey should be conducted to define common exhaust stubs used in turbo-machinery applications which contain non-symmetric gas turbine exhaust ducts. Secondly, model them numerically as in the current work and correlate the pressure losses.

2. The correlation produced from the current work serves as a guide for designers to produce more efficient non-symmetric gas turbine exhaust ducts. To be able to accurate predict the total pressure loss will require that the current CFD work be calibrated to experimental data. 


\section{Bibliography}

[1] G. Sovran and E. Klomp, "Experimentally determined optimum geometries for rectilinear diffusers with rectangular, conical , or annular cross-section," Fluid Mechanics of Internal Flows, pp. 270-319, 1967.

[2] A. McDonald and R. Fox, "An experimental investigation of incompressible flow in conical diffusers," ASME Paper, no. 65-FE-25, 1965.

[3] J. H. G. Howard, A. B. Thornton-Trump, and H. J. Henseler, "Performance and flow regimes for annular diffusers," ASME Paper, no. 67-WA/FE-21, 1967.

[4] D. Japikse and N. C. Baines, Diffuser Design Technology. Concepts ETI, Inc., 1998.

[5] A. Elkersh, A. Elgammal, and N. Maccallum, "An experimental investigation of the performance of equiangular annular diffusers with swirled flow," Proceedings of the Institution of Mechanical Engineers, vol. 199, no. C4, pp. 293-297, 1985.

[6] A. Klein, "Characteristics of combustor diffusers," Prog Aerospace Sci., vol. 31, pp. 171-271, 1995.

[7] D. Japikse, "Correlation of annular diffuser performance with geometry, swirl, and blockage," Proceedings of the 11th Thermal and Fluids Analysis Workshop, pp. 107118,2000 .

[8] M. Cunningham, Flow in Non-Symmetric Gas Turbine Exhaust Ducts. PhD thesis, Queen's University, 2002. 
[9] A. MacDonald, R. Fox, and R. Van Dewoestine, "Effects of swirling inlet flow on pressure recovery in conical diffusers," AIAA Journal, vol. 9, no. 10, pp. 2014-2018, 1971.

[10] Y. Senoo, N. Kawaguchi, and T. Nagata, "Swirl flow in conical diffusers," Bulletin of the JSME, vol. 21, no. 151, pp. 112-119, 1978.

[11] F. Dolan and P. J. Runstadler, "Pressure recovery performance of conical diffusers at high subsonic mach numbers," NASA CR-2299, 1973.

[12] V. K. Sharan, "An exponential investigation of the behavior of conical diffusers in turbulent flow," Journ Appl Math and Phys, vol. 27, 1976.

[13] J. Livesey and A. Odukwe, "Some effects of pipe flow generated entry conditions on the performance of straight walled conical diffusers with high subsonic entry mach number," Israel Journal of Technology, vol. 11, no. 4, pp. 217-222, 1973.

[14] D. Japikse, "A new diffuser mapping technique, part 1: Studies in component performance," Transactions of the ASME, vol. 108, pp. 148-156, 1986.

[15] A. Klein, "Review: Effects of inlet conditions on conical diffuser performance," ASME, Transactions, Journal of Fluids Engineering, vol. 103, pp. 250-257, 1981.

[16] S. Dovzhik and V. Kartavenko, "Measurement of the effect of flow swirl on the efficiency of annular ducts and exhaust nozzles of axial turbomachines," Fluid Mechanics - Soviet Research, vol. 4, no. 4, pp. 156-172, 1975.

[17] E. Klomp, "Performance of straight-walled annular diffusers with swirling flow," Aeronautical Journal, vol. 101, no. 1010, pp. 467-480, 1997.

[18] R. Coladipietro, J. Schneider, and K. Sridhar, "Effects of inlet flow conditions on the performance of equiangular annular diffusers," Transactions of the CSME, vol. 3, no. 2, pp. $75-82,1975$.

[19] S. Stevens and G. Williams, "The influence of inlet conditions on the performance of annular diffusers," ASME, Transactions, Journal of Fluids Engineering, vol. 102, pp. 357-363, 1980. 
[20] M. Loka, M. Robichaud, W. Di Bartolomeo, D. Loe, and D. Sowers, "Aerodynamic optimization of the exhaust-ejector system on the tiltrotor using navier-stokes analysis," paper AIAA 2000-0986, AIAA, 2000.

[21] R. K. Roy, Design of experiments using the Taguchi approach : 16 steps to product and process improvement. Wiley, 2001.

[22] D. Wyatt, "Analysis of errors introduced by several methods of weighting nonuniform duct flows," Tech. Rep. TN 3400, National Advisory Committee on Aeronautics, 1955.

[23] C. Rhie and W. Chow, "A numerical study of the turbulent flow past an isolated airfoil with trailing edge separation," AIAA Paper 82-0998, 1982.

[24] F. Menter, "Zonal two equation $k$ - $\omega$ turbulence models for aerodynamic flows," $A I A A$ Paper 93-2906, 1993.

[25] R. J. Schalkoff, Artificial Neural Networks. McGraw-Hill, 1997.

[26] W. P. Silva and C. M. D. P. S. Silva, LAB Fit Curve Fitting Software (Nonlinear Regression and Treatment of Data Program) V 7.2.42, 1999-2008. 